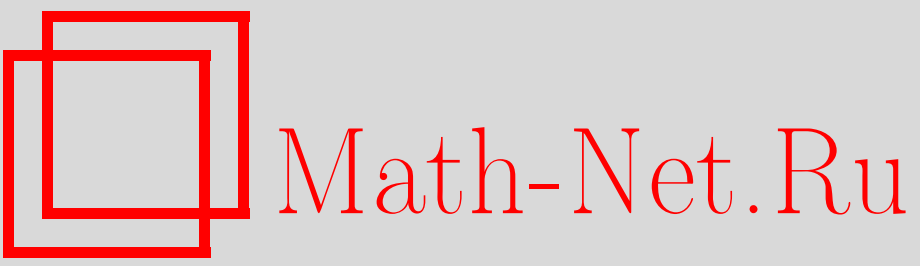

С. И. Адян, В. Г. Дурнев, Алгоритмические проблемы для групп и полугрупп, УМH, 2000, том 55, выпуск 2, 3-94

DOI: https://doi.org/10.4213/rm267

Использование Общероссийского математического портала Math-Net.Ru подразумевает, что вы прочитали и согласны с пользовательским соглашением

http://www.mathnet.ru/rus/agreement

Параметры загрузки:

IP: 44.207 .124 .84

26 апреля 2023 г., 06:47:21 


\title{
АЛГОРИТМИЧЕСКИЕ ПРОБЛЕМЫ ДЛЯ ГРУПП И ПОЛУГРУПП
}

\author{
С.И. Адян, В.Г. Дурнев
}

\begin{abstract}
В статье дается подробньй обзор результатов по основным алгоритмическим проблемам теории групп и полугрупп: проблемам равенства, изоморфизма, распознавания свойств и другим алгоритмическим вопросам, связанным с этими проблемами. Известные теоремы Маркова-Поста, П. С. Новикова, Адяна-Рабина, Хигмана, Магнуса, Линдона изложены с полными доказательствами. Как правило, приводимые в обзоре доказательства этих теорем существенно проще тех, которые давались авторами теорем в их оригинальных работах. В начале статьи для полноты приводится доказательство результата о неразрешимости проблемы остановки для машин Тьюринга, на котором основывается неразрешимость проблемы равенства для полугрупп. Особое внимание уделено также простейшим примерам полугрупп с неразрешимой проблемой равенства. Подробно изложено доказательство весьма примечательного результата Р. Линдона о разрешимости проблемы равенства в классе групп, задаваемых системой определяющих соотношений с условием, что максимальное взаимное наложение определяющих слов строго меньше $1 / 5$ длины самих этих слов, в то время как при замене в этом условии строгого неравенства на нестрогое уже возникает возможность неразрешимости. Изложено также доказательство аналогичного результата для конечно определенных полугрупп, где соответствующая точная граница равна $1 / 2$.

Библиограффия: 110 названий.
\end{abstract}

\section{СОДЕРЖАНИЕ}

1. Алгоритмы и машины Тьюринга …............................... 4

2. Неразрешимость проблемы равенства для полугрупп ............... 9

3. Некоторые классы полугрупп с разрешимой проблемой равенства ... 22

4. Полугруппы с одним определяюшим соотношением $\ldots . \ldots \ldots \ldots \ldots \ldots \ldots . . . . . .27$

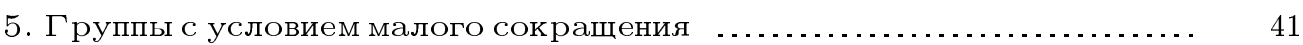

6. Неразрешимость проблемы равенства для групп $\ldots \ldots \ldots \ldots \ldots \ldots \ldots \ldots . \quad 52$

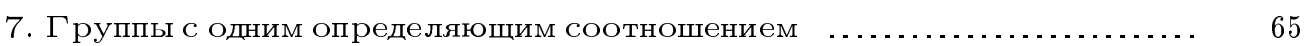

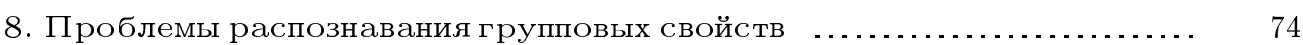

9. Проблема вхождения и некоторые результаты о вложении . . . . . . . . . . 78

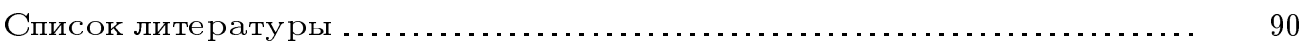

Работа выполнена при финансовой поддержке Российского фонда фундаментальных исследований и INTAS (грант № 97-1259). 


\section{1. Алгоритмы и машины Тьюринга}

Под алгоритмом в интуитивном смысле мы понимаем точное предписание, определяюшее вычислительный процесс, который ведет от исходных данных, варьируемых в некотором заданном множестве, к искомому результату. Этот вычислительньй процесс должен быть конечным и должен однозначно определяться заданием предписания и конкретного исходного данного. Иногда назьвают алгоритмом и сам выгислительный процесс, определяемый данным предписанием.

Под алгоритмической проблемой понимается задача построения единого алгоритма для решения заданной массовой задачи, т.е. бесконечной серии однотипных вопросов, зависящих от каких-то параметров. В случае, когда искомый алгоритм невозможен, говорят, что данная алгоритмическая проблема неразрешима. Неразрешимость алгоритмической проблемы, конечно, вовсе не означает, что какая-то из задач рассматриваемой серии неразрешима. Это означает лишп, что нельзя указать единьй метод решения всех задач данной серии. Возможность решения каждой из них своим способом при этом не исключается.

Предписание, задающее алгоритм, должно быть конечным, и искомый результат должен получаться через конечное число шагов работы алгоритма. Оно должно быть настолько четким, чтобы однозначно определять весь вычислительньй процесс от начальных данных до результата. Это означает, что процесс осушествляется вычислителем (будь то человек или машина) чисто механически, т.е. без привлечения каких-либо творческих элементов, и может быть воспроизведен с тем же результатом другим вычислителем и в другое время. Иначе говоря, для выполнения вычислительного процесса не требуется никакого творческого усилия.

Вплоть до 30-х годов ХХ века математики обходились неточньм интуитивньм понятием алгоритма. Возникавшие алгоритмические проблемы решались указанием соответствуюших разрешаюших процедур. При этом каждый раз, когда конкретный алгоритм для решения той или иной серии однотипных задач был построен, не возникало никаких сомнений в том, что указанная процедура является алгоритмом.

К началу ХХ века в математике стали появляться глубокие алгоритмические проблемы, которые не удавалось решить, несмотря на усилия многих квалифицированных математиков. Начали возникать сомнения в возможности их положительного решения. Но для доказательства невозможности алгоритма, дающего решение той или иной серии задач, следовало сначала точно математически определить, какой смысл вкладьвается в понятие “алгоритм". Такое уточнение определения понятия алгоритма стало возможньм благодаря развитию математической логики в начале ХХ века. Оно было получено в середине тридцатых годов в работах К. Геделя, Д. Эрбрана, А. Черча, Э. Поста и А. Тьюринга почти одновременно в двух внешне совершенно различных формах: в виде точного математического описания класса вычислимых функций натурального аргумента (частично рекурсивные и рекурсивные функции) и в виде точного математического определения класса вычислительных процессов, реализуемых механически абстрактным выгислителем (машины Тьюринга). Вскоре было установлено, что эти два уточнения понятия алгоритма по существу эквивалентны друг другу, т.е. они взаимозаменяемы. Это дало основание американскому математику А. Черчу в 1936 году [29] сформулировать следующий тезис. 
ТЕЗИС ЧЕРчА. Всякий алгоритм в интуитивном смысле с точки зрения его вычислительных возможсностей эквивалентен некоторому алгоритму в уточненном смысле.

Появление точного понятия алгоритма позволило установить неразрешимость многих алгоритмических проблем. Сначала неразрешимые алгоритмические проблемы были отмечены в самой теории алгоритмов, затем в математической логике. Позже было доказано, что они есть и среди известных задач, поставленных в математике ранее.

Наиболее естественными объектами, используемыми в качестве исходных данных и результатов работы алгоритмов, являются слова в том или ином алфавите. Алфавитом называется любое непустое множество символов, называемых буквами алфавита. Конечная последовательность, составленная из записанных друг за другом букв алфавита $\mathscr{A}$, называется словом в алфавите $\mathscr{A}$. Мы рассматриваем также и пустое слово, которое обозначается через $\Lambda$ или 1 . От алфавита требуется, чтобы была исключена возможность “разночтения" слов, т.е. каждое слово в этом алфавите должно иметь единственное разбиение на составляюшие его буквы.

Множество всех слов в алфавите $\mathscr{A}$ будем обозначать через $\mathscr{A}^{*}$. Графическое совпадение слов $X$ и $Y$ обозначается через $X \mp Y$. Длину слова $X$ будем обозначать через $|X|$. Символом $\rightleftharpoons$ обозначается равенство по определению. Говорят, что слово $E$ входит в слово $X$, если можно указать такие слова $R$ и $Q$, что $X \mp R E Q$. Если при этом слово $R$ (слово $Q$ ) пусто, то слово $E$ называется началом (соответственно, концом) слова $X$.

Если $X$ - некоторое слово, а $n$ - натуральное число, то через $X^{n}$ обозначается результат приписывания друг к другу $n$ экземпляров слова $X$. При этом полагаем $X^{0} \rightleftharpoons \Lambda$.

Машина Тъюринга $T$ имеет ленту, разбитую на конечное число ячеек, в которые можно записывать символы из данного набора $\left\{a_{0}, a_{1}, \ldots, a_{k}\right\}$, назьваемого алфавитом ленточных символов. По техническим причинам удобно считать, что среди этих символов имеется так называемый пустой символ, который мы будем обозначать $a_{0}$. Ячейки ленты упорядочены слева направо. Мы будем предполагать, что лента машины потенииально бесконечна в обе сторонь. Это означает, что в процессе работы машины Тьюринга разрешается добавлять новые пустые ячейки как к правому, так и к левому концу ленты (мы считаем, что в момент добавления в них записан пустой символ $\left.a_{0}\right)$. В принципе можно было бы считать ленту бесконечной, но потребовать, чтобы в каждый момент времени лишш конечное число ее ячеек содержало непустые символы.

У машины Тьюринга $T$ имеется также головка, которая в каждьй момент времени обозревает некоторую ячейку и сама находится в одном из данного множества внутренних состояний

$$
\left\{q_{0}, q_{1}, \ldots, q_{l}\right\}
$$

Состояние $q_{0}$ назьвается заключительным состоянием.

Работа машины Тьюринга состоит из отдельных тактов, выполняемых согласно заданной программе. Программа $P_{T}$ машины Тьюринга $T$ - это конечньй набор команд следующих трех типов:

$$
q_{i} a_{j}\left|q_{r} a_{t}, \quad q_{i} a_{j}\right| q_{r} R, \quad q_{i} a_{j} \mid q_{r} L .
$$


При этом каждой паре символов $\left(q_{i}, a_{j}\right)$ при $i>0$ соответствует ровно одна команда, в которой перед | стоит слово $q_{i} a_{j}$. Эту команду мы будем обозначать через $P_{T}(i, j)$. Если в рассматриваемый момент времени головка машины Тьюринга находится в состоянии $q_{i}$ при $i>0$, а в обозреваемой ею ячейке находится символ $a_{j}$, то машина выполняет команду $P_{T}(i, j)$ следуюшим образом.

1) Если команда имеет вид

$$
q_{i} a_{j} \mid q_{r} a_{t}
$$

то в обозреваемой ячейке символ $a_{j}$ заменяется на символ $a_{t}$ и головка переходит в состояние $q_{r}$.

2) Если команда имеет вид

$$
q_{i} a_{j} \mid q_{r} R \text { или } q_{i} a_{j} \mid q_{r} L,
$$

то, не меняя символ $a_{j}$, головка переходит в состояние $q_{r}$ и сдвигается в соседнюю справа (соответственно, слева) ячейку. При этом если считьвающая головка находилась в самой правой (левой) ячейке, то к ленте пристраивается новая пустая ячейка.

Если в результате выполнения некоторой команды головка машины приходит в заключительное состояние $q_{0}$, то машина останавливается, так как по определению ни одна команда программы не начинается с $q_{0}$.

Полное описание состояния машины Тьюринга в данньй момент времени задается словом вида $A q_{i} a_{j} B$, где $q_{i}$ - состояние головки в данньй момент, $a_{j}-$ символ, записанньй в обозреваемой ячейке, а $A$ и $B$ - слова, записанные левее и правее этой ячейки. Такое слово $A q_{i} a_{j} B$ называется полной конфигурацией или просто конфиәураиией, описывающей состояние машины Тьюринга в данньй момент. При этом будем говорить, что машина $T$ находится в конфигурации $A q_{i} a_{j} B$.

Пусть конфигурация $K_{T}$, в которой машпна $T$ находится в данньй момент времени, имеет вид $K_{T}=A q_{i} a_{j} B$, где $1 \leqslant i \leqslant l, 0 \leqslant j \leqslant k$.

Опишем конфигурацию $K_{T}^{\prime}$, в которую машина перейдет в результате применения соответствуюшей команды $P_{T}(i, j)$ из программы $P_{T}$.

1) Если команда имеет вид $q_{i} a_{j} \mid q_{r} a_{t}$, то $K_{T}^{\prime}=A q_{r} a_{t} B$.

2) Пусть выполняемая команда имеет вид $q_{i} a_{j} \mid q_{r} R$.

Если слово $B$ непусто, т.е. $B=a_{s} B^{\prime}$ при некотором $a_{s}$, то $K_{T}^{\prime}=A a_{t} q_{r} a_{s} B^{\prime}$.

Если же слово $B$ пусто, то $K_{T}^{\prime}=A a_{t} q_{r} a_{0}$.

3) Если вьполняемая команда $P_{T}(i, j)$ имеет вид $q_{i} a_{j} \mid q_{r} L$, то искомая конфигурация $K_{T}^{\prime}$ получается симметричным образом.

Среди внутренних состояний машины Тьюринга $T$ часто выделяют еще и начальное состояние $q_{1}$. Если машина Тьюринга $T$, начав работать в конфигурации $q_{1} a_{0} E$, через конечное число тактов работы перейдет в конфигурацию вида $D q_{0} B$, то слово $D B$ назьвается результатом применения к слову $E$ алгоритма, определяемого машиной Тьюринга $T$. В противном случае мы говорим, что этот алгоритм к слову $E$ не применим. Иначе говоря, мы можем считать, что машина $T$ вычисляет некоторую словарную функцию $f(x)$, значением которой при $x=E$ является слово $D B$, причем эта функция определена только для тех $E$, при которых машина остановится.

Машины Тьюринга можно использовать для вычисления функций, аргументы и значения которых пробегают слова в данном алфавите, содержащемся в алфавите ленточных символов. В частности, речь может идти о вычислении функций от натурального аргумента. При этом натуральное число $n$ представляется словом $\bar{n}$ длины $n$ в однобуквенном алфавите $\{1\}$. Мы говорим, что машина Тьюринга $T$ вычисляет данную 
функцию $f(n)$, если начав работу в состоянии $q_{1} a_{0} \bar{n} a_{0}$, она останавливается в том и только том случае, если $f(n)$ определено и при этом заключительное состояние имеет вид $q_{0} a_{0} \overline{f(n)} a_{0}$. Числовая функция называется вычислимой по Тьюрингу, если существует машина Тьюринга, вычисляющая эту функцию. Следующее утверждение равносильно тезису Черча.

ТЕЗИС ЧЕРчА В фОРме ТЬЮРИНГА. Если некоторая функиия вычислима с помощъю алгоритма в интуитивном смысле, то она вычислима по Тьюрингу.

Простейшим примером алгоритмически неразрешимой проблемы для машин Тьюринга является проблема распознавания самоприменимости, которая представляет собой частньй случай проблемы остановки.

ПРОБЛЕМА ОСТАНОВКИ. Найти алгоритм, позволяющий по произвольной машине Тьюринга $T$ и произвольной ее начальной конфигурачии $E_{1} q_{1} E_{2}$ определить, придет ли машина $T$ в заключительное состояние $q_{0}$, если начнет работу в этой конфигурации.

ПРОБЛЕМА РАСПОЗНАВАНИЯ САМОПРИМЕНИМОСТИ. Найти алгоритм, который позволяет определить по произвольной машине Тъюринга T, применима ли она к коду своего номера, т.е. к слову $\overline{n(T)}$.

Для доказательства неразрешимости этих проблем мы используем нумерацию машин Тьюринга, вычисляюших функции натурального аргумента.

Предполагая, что символ $a_{1}$ обозначает 1 , мы можем считать, что при вычислении функций натурального аргумента все ленточные символы машин Тьюринга берутся из счетного алфавита

$$
\left\{a_{0}, a_{1}, \ldots, a_{k}, \ldots\right\}
$$

а внутренние состояния - из счетного алфавита

$$
\left\{q_{0}, q_{1}, \ldots, q_{l}, \ldots\right\}
$$

Пусть $T$ - произвольная машина Тьюринга с программой $P_{T}$, в которой используются ленточные символы $a_{0}, a_{1}, \ldots, a_{k}$ и внутренние состояния $q_{0}, q_{1}, \ldots, q_{l}$. Для упрошения записи программы машины Тьюринга договоримся обозначать символ $L$ через $a_{-1}$, а символ $R$ - через $a_{-2}$. Можно считать, что каждая команда машины Тьюринга $T$ имеет вид

$$
q_{i} a_{j} \mid q_{r} a_{t}
$$

где $t=-2,-1,0,1, \ldots, k$.

Пусть $p_{1}, p_{2}, \ldots, p_{n}, \ldots$ - нумерация всех простых чисел в порядке возрастания. Занумеруем произвольньмм образом все команды программы данной машины Тьюринга $T$ :

$$
\begin{gathered}
q_{i_{1}} a_{j_{1}} \mid q_{r_{1}} a_{t_{1}}, \\
q_{i_{2}} a_{j_{2}} \mid q_{r_{2}} a_{t_{2}}, \\
\ldots, \\
q_{i_{m}} a_{j_{m}} \mid q_{r_{m}} a_{t_{m}} .
\end{gathered}
$$


Программу машины Тьюринга $T$ закодируем натуральным числом

$$
n(T) \rightleftharpoons p_{1}^{i_{1}} p_{2}^{j_{1}} p_{3}^{r_{1}} p_{4}^{t_{1}+2} p_{5}^{i_{2}} p_{6}^{j_{2}} p_{7}^{r_{2}} p_{8}^{t_{2}+2} \ldots p_{4 m-3}^{i_{m}} p_{4 m-2}^{j_{m}} p_{4 m-1}^{r_{m}} p_{4 m}^{t_{m}+2},
$$

которое будем называть номером машины Тьюринга $T$.

Очевидно, данная машина Тьюринга может иметь несколько номеров, но не каждое натуральное число является номером какой-то машины Тьюринга. Легко построить алгоритм, который по произвольному натуральному числу $n$ определяет, является ли оно номером некоторой машины Тьюринга, и в случае положительного ответа выдает программу этой машины.

ТЕОРема 1.1. Невозможен алгоритм, распознающий по любому натуральному числу п, является ли оно номером самоприменимой машинь Тьюринга.

ДокАЗАТЕльство. Допустим, что сушествует алгоритм распознавания самоприменимости. Обозначим через $M_{0}$ множество всех номеров самоприменимых машин Тьюринга. Тогда в силу тезиса Черча существует машина Тьюринга $T_{0}$, выгисляющая характеристическую функцию множества $M_{0}$, т.е. функцию, равную 1 на элементах из $M_{0}$ и 0 - на элементах дополнения. Напомним, что машина $T_{0}$ при любой начальной конфигурации вида $q_{1} a_{0} \bar{n}$ останавливается в заключительном состоянии вида $q_{0} a_{0} a_{1} a_{0}$, если $n \in M_{0}$, и $-q_{0} a_{0} a_{0}$ в противном случае.

В программе $P_{T_{0}}$ заменим все вхождения символа $q_{0}$ новым символом $q_{r}$, который не входит в команды машины $T_{0}$, и добавим следуюшие новые команды:

$$
q_{r} a_{0}\left|q_{r+1} R, q_{r+1} a_{0}\right| q_{0} a_{0}, q_{r+1} a_{1} \mid q_{r+1} a_{1},
$$

учитывая, что $q_{r+1}$ также не входит в команды машины $T_{0}$. Очевидно, построенная таким образом машина $T_{1}$ применима к слову $\bar{n}$ в том и только том случае, если $n \in C M_{0}$. Пусть $n_{1}$ есть номер машины $T_{1}$. Если $n_{1} \in C M_{0}$, то по определению множества $M_{0}$ машина $T_{1}$ не применима к слову $\overline{n_{1}}$, что противоречит определению машины $T_{1}$. Если же $n_{1} \in M_{0}$, то по определению $M_{0}$ машина $T_{1}$ применима к слову $\overline{n_{1}}$, что невозможно, так как по построению машина $T_{1}$ не применима к словам $\bar{n}$ при $n \in M_{0}$. Полученное противоречие завершает доказательство теоремы.

Из доказанной теоремы сразу следует алгоритмическая неразрешимость проблемы остановки для машин Тьюринга.

Теорема 1.2. Можно построить машину Тьюринга $T_{0}$, для которой невозможен алгоритм, решающий для нее проблему остановки.

ДокАЗАТЕЛЬСТво. В качестве искомой машины Тьюринга $T_{0}$ можно взять машину, перечисляющую множество натуральных чисел $M_{0}$, т.е. такую машину $T_{0}$, которая применима к слову $\bar{n}$ тогда и только тогда, когда натуральное число $n$ является номером некоторой самоприменимой машины Тьюринга. Явное построение такой машины сопряжено с технически сложными конструкциями. Мы же здесь ограничимся обоснованием того, как ее существование следует из тезиса Черча в форме Тьюринга. В самом деле, в силу этого тезиса нам достаточно убедиться в существовании алгоритма $\mathfrak{A}$, который применим к словам $\bar{n}$ тогда и только тогда, когда натуральное число $n$ является номером некоторой самоприменимой машины Тьюринга. Укажем, как строится такой алгоритм $\mathfrak{A}$. По произвольному натуральному числу $n$, разложив его 
в произведение простых множителей и проанализировав это разложение, определяем, является ли $n$ номером некоторой машины Тьюринга. Если число $n$ является номером некоторой машины Тьюринга, то мы найдем программу этой машины $T_{n}$ и передадим ей управление в начальной конфигурации $q_{1} a_{0} \bar{n} a_{0}$. В этом случае наш алгоритм завершает работу тогда и только тогда, когда остановится машина Тьюринга $T_{n}$, а это случится в точности тогда, когда $n$ есть номер самоприменимой машины Тьюринга. Если же число $n$ не является номером никакой машины Тьюринга, то наш алгоритм $\mathfrak{A}$ не останавливается. Теорема 1.2 доказана.

\section{2. Неразрешимость проблемы равенства для полугрупп}

Универсальньй способ задания полугрупп с помощью порождающих элементов и определяюших соотношений был предложен в 1914 году А. Туэ в работе [101]. Там же он впервые сформулировал алгоритмическую проблему распознавания равенства слов для конечно определенных полугрупп, т.е. таких заданий, где число порождающих и число определяющих соотношений конечно.

Очевидно, множество $\mathscr{A}^{*}$ всех слов в алфавите $\mathscr{A}$ образует свободную полугрупшу относительно бинарной операции приписывания одного слова к другому. При этом пустое слово играет роль единицы и обозначается через 1.

Пусть задан произвольньй алфавит $\mathscr{A}$ и некоторое множество $\mathscr{D}$ упорядоченных пар слов в алфавите $\mathscr{A}$. По сложившейся традиции упорядоченную пару слов $\langle A, B\rangle$ при задании полугруппы будем записьвать в виде равенства $A=B$. Эти равенства, называемые определяющими соотношениями, определяют на множестве всех слов некоторое отношение әквивалентности, факторизация по которому переводит свободную полугруппу $\mathscr{A}^{*}$ в полугруппу, заданную определяюшими соотношениями множества $\mathscr{D}$ и обозначаемую через

$$
\Pi=\langle\mathscr{A} \mid \mathscr{D}\rangle .
$$

Отношение равенства слов в таким образом заданной полугруппе П определяется следующим образом.

Левым әлементарным преобразованием, отвечаюшим определяющему соотношению $A=B$, называется любой переход вида

$$
U A V \rightarrow U B V
$$

где $U$ и $V$ - произвольные слова.

Правыми әлементарными преобразованиями называются переходы вида

$$
U B V \rightarrow U A V
$$

В дальнейшем запись $P \rightarrow Q$ будет обозначать, что слово $Q$ получается из слова $P$ с помощью одного элементарного преобразования рассматриваемой полугруппь.

Два слова $P$ и $Q$ в алфавите $\mathscr{A}$ назьваются әквивалентными в полугруппе $\Pi$, если либо $P=Q$, либо сушествует цепочка элементарных преобразований вида

$$
P=P_{0} \rightarrow P_{1} \rightarrow \cdots \rightarrow P_{k}=Q
$$

(записывается $P=Q$ в П). 
Очевидно, определенное таким образом отношение эквивалентности согласовано с операцией умножения слов, т.е. если $P_{1}=Q_{1}$ и $P_{2}=Q_{2}$, то $P_{1} P_{2}=Q_{1} Q_{2}$.

Элементами полугрупшы П являются классы эквивалентности $[X]$, состояшие из всех слов, эквивалентных слову $X$. Полугруппа П представляет собой множество всех классов эквивалентности с ассоциативной операцией $[X] \cdot[Y] \rightleftharpoons[X Y]$, результат которой не зависит от выбора представителей $X, Y$ классов эквивалентности.

Очевидно, если добавить к множеству $\mathscr{A}$ новую букву $b$ и к соотношениям - некоторое равенство $b=W$, где $W$ есть слово в алфавите $\mathscr{A}$, то получим новое задание той же полугруппы. Также очевидно, что два задания полугрупп $\Pi_{1}=\left\langle\mathscr{A} \mid \mathscr{D}_{1}\right\rangle$ и $\Pi_{2}=\left\langle\mathscr{A} \mid \mathscr{D}_{2}\right\rangle$, определяют одну и ту же полугруппу, если они удовлетворяют условию:

любое равенство из $\mathscr{D}_{2}$ выполнено в $\Pi_{1}$ и

любое равенство из $\mathscr{D}_{1}$ выполнено в $\Pi_{2}$.

Если множества $\mathscr{A}$ и $\mathscr{D}$ конечны, то задание полугруппы П $(\mathscr{A}, \mathscr{D})$ может быть записано в виде

$$
\left\langle a_{1}, \ldots, a_{n} \mid A_{1}=B_{1}, \ldots, A_{m}=B_{m}\right\rangle .
$$

Для того чтобы полугруппа с единицей была группой достаточно, чтобы в ней имелись обратные элементы для всех порождающих. Задание групп с помощью образующих и определяющих соотношений осуществляется следующим образом.

К данному исходному алфавиту

$$
\mathscr{A}=\left\{a_{1}, a_{2}, \ldots, a_{i}, \ldots\right\}
$$

добавляется алфавит “двойников”, называемых обратными степенями образующих,

$$
\mathscr{A}^{-1}=\left\{a_{1}^{-1}, a_{2}^{-1}, \ldots, a_{i}^{-1}, \ldots\right\} .
$$

Полученное объединение называется групповым алфавитом. При задании любой группы с порождающими (1) для обеспечения того, чтобы буквы $a_{i}^{-1}$ были обратными для букв $a_{i}$, служат так называемые тривиальные определяющие соотношения, имеющие вид

$$
a_{i}^{-1} a_{i}=1, \quad a_{i} a_{i}^{-1}=1
$$

Для задания группы к тривиальным соотношениям (2) добавляются определяющие соотношения в групповом алфавите, которые в данном случае всегда можно записывать в виде равенств

$$
A_{i}=1 \quad(i=1,2, \ldots, m) .
$$

Так как добавление обратных степеней образуюших (1) и тривиальных соотношений (2) однозначно определяется заданием множества порождаюших (1), то обычно говорят о группе $G$, заданной порождаюшими (1) и определяюшими соотношениями (3), и записывают это задание в виде

$$
\left\langle\left\langle a_{1}, a_{2}, \ldots, a_{n} \mid A_{1}=1, A_{2}=1, \ldots, A_{m}=1\right\rangle\right\rangle .
$$


Соответствуюшие тривиальным соотношениям (2) элементарные преобразования

$$
U a_{i}^{-1} a_{i} V \rightarrow U V \quad \text { и } U a_{i} a_{i}^{-1} V \rightarrow U V
$$

назьваются сокращениями букв $a_{i}$ и ${a_{i}^{-1}}^{-1}$ а обратные им преобразования называются вставками этих букв.

Слово $X$ назьвается несократимым, если оно не содержит вхождений подслов вида $a_{i} a_{i}^{-1}$ или вида $a_{i}^{-1} a_{i}$. Очевидно, в любой групе произвольное слово можно преобразовать в равное ему несократимое слово, используя только преобразования (4).

Напомним, что элементами группы $G$ являются классы эквивалентных слов в групповом алфавите, которые однозначно определяются любым своим представителем.

Если

$$
X=a_{i_{1}}^{\sigma_{1}} a_{i_{2}}^{\sigma_{2}} \ldots a_{i_{k-1}}^{\sigma_{k-1}} a_{i_{k}}^{\sigma_{k}},
$$

где $\sigma_{j}= \pm 1(j=1,2, \ldots, k)$, то слово

$$
a_{i_{k}}^{-\sigma_{k}} a_{i_{k-1}}^{-\sigma_{k-1}} \ldots a_{i_{2}}^{-\sigma_{2}} a_{i_{1}}^{-\sigma_{1}}
$$

назьвается обратным к слову $X$ и обозначается через $X^{-1}$. Очевидно, слова $X$ и $X^{-1}$ определяют взаимно обратные элементы группы.

Если множество определяющих соотношений пусто, то мы получаем свободную грynny с $n$ порождающими. Она обозначается через $F_{n}$. Число $n$ называется ее $p a н-$ гом.

Проблема распознавания равенства слов для полугрупп, сформулированная А. Туэ в 1914 году [101], состоит в следующем.

Построить алгоритм, позволяющий для произвольной полугруппь П и любьх двух слов $U$ и $V$ в ее алфавите определить, представляют ли они один и тот же әлемент этой полугруппь, т.е. эквивалентны ли они в П.

Для конечно определенных групп аналогичная проблема равенства была сформулирована М. Дэном двумя годами раныше в работе [31]. Ее иногда еще называют проблемой тождества.

Проблему равенства можно ставить и для каждой полугруппы или группы в отдельности. При этом мы получим частную проблему равенства для фиксированной полугруппы (группы).

Конечно, Дэн и Туэ не предполагали, что искомый алгоритм может не существовать. Они просто ставили задачу построения алгоритма для решения важной проблемы, связанной с универсальным способом задания полугрупп и групп. Более того, появившиеся только в середине ХХ века доказательства неразрешимости этих проблем оказались неожиданными для широкого круга математиков. Ниже мы приведем полные доказательства этих фундаментальных результатов А. А. Маркова, Э. Поста, П. С. Новикова, В. В. Буна и других авторов в современном упрощенном изложении.

В 1947 году А. А. Марков [57] и Э. Пост [84] независимо доказали существование конечно определенных полугруп с неразрешимой проблемой равенства слов. Это был первый в истории математики пример неразрешимой проблемы, которая была сформулирована задолго до появления в науке точного определения понятия алгоритма. Мы приведем здесь доказательство этого результата, существенно более простое, чем 
оригинальные доказательства самих авторов. Оно основано на доказанной вьше неразрешимости проблемы остановки для машины Тьюринга $T_{0}$.

Пусть дана произвольная машина Тьюринга $T$ с внешним алфавитом $A_{T}=\left\{a_{0}, a_{1}\right.$, $\left.\ldots, a_{n}\right\}$, алфавитом внутренних состояний $Q_{T}=\left\{q_{0}, q_{1}, \ldots, q_{m}\right\}$ и программой $P_{T}$. По этой программе $P_{T}$ мы естественным образом построим конечно определенную полугруппу $S(T)$, заданную множеством образующих элементов

$$
\left\{a_{0}, a_{1}, \ldots, a_{n}, q_{0}, q_{1}, \ldots, q_{m}, h\right\}
$$

и некоторым множеством определяющих соотношений $\mathscr{R}(S(T))$, которая строится по программе $P_{T}$.

Для каждой команды $P_{T}(i, j)$ из $P_{T}$ мы включаем в $\mathscr{R}(S(T))$ соотношения определенного ниже вида.

I. Если $P_{T}(i, j)$ имеет вид $q_{i} a_{j} \mid q_{r} a_{t}$, то включаем в $\mathscr{R}(S(T))$ одно соотношение $q_{i} a_{j}=q_{r} a_{t}$.

II. Если команда $P_{T}(i, j)$ имеет вид $q_{i} a_{j} \mid q_{r} L$, то включаем все соотношения вида $a_{t} q_{i} a_{j}=q_{r} a_{t} a_{j}$ при $t=0, \ldots, n$ и еше одно соотношение $h q_{i} a_{j}=h q_{r} a_{0} a_{j}$.

III. Если команда $P_{T}(i, j)$ имеет вид $q_{i} a_{j} \mid q_{r} R$, то включаем все соотношения вида $q_{i} a_{t} a_{j}=a_{t} q_{r} a_{j}$ при $t=0, \ldots, n$ и еше одно соотношение $q_{i} a_{j} h=a_{j} q_{r} a_{0} h$.

IV. Кроме того, добавим в $\mathscr{R}(S(T))$ еше $h q_{0} h=q_{0}$ и все соотношения видов $q_{0} a_{t}=q_{0}$ и $a_{t} q_{0}=q_{0}$.

Каждой конфигурации $X=E_{1} q_{i} E_{2}$ машины $T$ ставим в соответствие слово $h E_{1} q_{i} E_{2} h$, которое будем называть словом Поста.

Заметим, что элементарные преобразования типов I, II, III любое слово Поста переводят в слово Поста. Следуюшая лемма очевидна.

Лемма 2.1. Машина Тьюринга $T$ переводит за один такт работьл конфигурацию $X$ в конфигурацию $Y$ в том и только том случае, если слово Поста $Х$ һ переводится в слово Поста $h Y h$ одним левым әлементарным преобразованием, соответствующим команде, применяемой машиной $T$.

ЛЕмма 2.2. Машина Тьюринга $T$, начав работать в конфигурации $X$, через конечное число тактов работы остановится тогда и только тогда, когда в полугруппе $S(T)$ выполняется равенство $h X h=q_{0}$.

ДокАЗАТЕльство. Пусть машина Тьюринга $T$, начав работать в конфигурации $X$, через конечное число тактов работы остановится, т.е. имеется конечная цепочка конфигураций $X, X_{1}, X_{2}, \ldots, X_{k-1}, X_{k}$, оканчивающаяся заключительной конфигурацией $X_{k}$. Тогда по лемме 2.1 имеем последовательность элементарных преобразований полугруппы $S(T)$

$$
h X h \rightarrow h X_{1} h \rightarrow h X_{2} h \rightarrow \cdots \rightarrow h X_{k-1} h \rightarrow h X_{k} h,
$$

где $h X_{k} h$ имеет вид $h Z_{1} q_{0} Z_{2} h$. Используя определяюшие соотношения из IV-й группы, получаем, что в полугруппе $S(T)$ вьполняется равенство $h X h=q_{0}$.

Предположим, теперь, что в полугруппе $S(T)$ вьполняется равенство $h X h=q_{0}$. Это значит, что существует цепочка элементарных преобразований

$$
h X h=Y_{0} \rightarrow Y_{1} \rightarrow Y_{2} \rightarrow \cdots \rightarrow Y_{k-1} \rightarrow Y_{k} \mp q_{0}
$$


Очевидно, в каждое слово $Y_{j}$ из последовательности (5) входит только одна $q$-буква. Рассмотрим кратчайшую цепочку элементарных преобразований вида (5), заканчивающуюся словом, содержащим $q_{0}$ :

$$
h X h=Y_{0} \rightarrow Y_{1} \rightarrow Y_{2} \rightarrow \cdots \rightarrow Y_{r-1} \rightarrow Y_{r}=Z_{1} q_{0} Z_{2} .
$$

В преобразованиях (6) определяюшие соотношения полугруппы $S(T)$ из IV-й группы не используются. Поэтому в (6) все слова являются словами Поста. Пусть

Покажем, что в цепочке (6) все преобразования левые. Допустим, что это не так.

$$
Y_{t} \rightarrow Y_{t+1}
$$

есть самое последнее правое элементарное преобразование в (6). Тогда $t+1<r$ и за преобразованием (7) должно следовать левое преобразование

$$
Y_{t+1} \rightarrow Y_{t+2}
$$

Так как среди левых частей определяющих соотношений типов I, II, III есть только одно слово $E$, содержащее данное двубуквенное подслово $q_{i} a_{j}$, то в $(8)$ может использоваться лишь то же самое соотношение, что и в (7). Более того, однозначно определено также и вхождение всего определяющего слова $E$ в слово $Y_{t+1}$. Поэтому получаем $Y_{t+2}=Y_{t}$, что противоречит условию минимальности последовательности (6). Следовательно, (6) состоит только из левых элементарных преобразований. Тогда по лемме 2.1 машина $T$ из конфигурации $X$ через конечное число шагов перейдет в заключительное состояние $q_{0}$ и остановится. Лемма 2.2 доказана.

Пусть $T_{0}$ - машина Тьюринга с неразрешимой проблемой остановки. Тогда из теоремы 1.2 и леммы 2.2 следует

Теорема 2.1 (А.А. Марков, Э. Пост). Невозможен алгоритм, определяющий для произвольного слова $X$ в алфавите образующих полугруппь $S\left(T_{0}\right)$, равно ли оно $q_{0}$ в полугруппе $S\left(T_{0}\right)$.

Заметим, что любая конечно определенная полугруппа вкладывается в полугруппу, заданную 2 порождаюшими и конечным числом определяюших соотношений. Для этого можно воспользоваться следующей подстановкой двубуквенных слов вместо всех исходных порождающих:

$$
a_{i} \rightarrow a b a^{i+1} b^{i+1} .
$$

Поэтому сушествует полугруппа с неразрешимой проблемой равенства, заданная двумя порождаюшими и конечным числом определяюших соотношений.

Наиболее простые (по общей длине задания) примеры полугрупп с неразрешимой проблемой распознавания равенства слов были построены в 1956 году независимо Г. С. Цейтиным [104] и Д. Скоттом [94]. Оба эти задания содержали 7 определяющих соотношений. Ниже мы приводим пример Цейтина с доказательством.

ТЕОРема 2.2 (Г. С. Цейтин [104]). Полугруппа Ц, заданная образующими $a, b, c$, $d$, е и определяющими соотношениями

$$
a c=c a, \quad a d=d a, \quad b c=c b, \quad b d=d b, \quad c e=e c a, \quad d e=e d b, \quad c c a=c c a e,
$$

имеет неразрешимую проблему равенства слов. 
ДокАЗАТЕльСТво. На самом деле будет доказана неразрешимость проблемы распознавания равенства в Ц произвольного слова $X$ некоторому фиксированному слову.

Следующая ниже основная лемма утверждает, что полугруппа Ц в некотором смысле универсальна для всех полугрупп $G$, имеющих задание специального вида.

Рассмотрим произвольную конечно определенную полугруппу $G$, имеюшую задание специального вида с пустыми правыми частями соотношений:

$$
\left\langle b_{1}, \ldots, b_{k} \mid K_{j}=1(j=1, \ldots, m)\right\rangle .
$$

Очевидно, это задание эквивалентно следующему:

$$
\left\langle b_{1}, \ldots, b_{k}, \alpha \mid \alpha=1,\left\{K_{j} \alpha=1 \mid j=1, \ldots, m\right\}\right\rangle .
$$

Индукцией по длине слова $X$ на словах в алфавите полугрупшы $G$ определим отображение $\tau(X)$ :

$$
\tau(\Lambda)=a, \quad \tau(X \alpha)=\tau(X) a, \quad \tau\left(X b_{j}\right)=\tau(X) b^{j} a \quad(j=1,2, \ldots, k) .
$$

ОПРЕДЕЛЕНИЕ. Для произвольного непустого слова $X$ через $[X$ ) (через $(X])$ будем обозначать слово, полученное в результате удаления из слова $X$ последней (соответственно, первой) буквы.

Очевидно, для любых двух слов $X$ и $Y$ в алфавите образуюших полугруппы $G$ выполняются равенства

$$
\begin{gathered}
\tau(X Y)=\tau(X)(\tau(Y)]=[\tau(X)) \tau(Y), \\
\tau(X)=a(\tau(X)]=[\tau(X)) a .
\end{gathered}
$$

Лемма 2.3. Если $P, Q-$ слова в алфавите полугруппь $G$, то

$$
\begin{aligned}
P=Q \text { в } G \Longleftrightarrow \tau(P)=\tau(Q) \text { в nолугpynne } \\
\qquad \mathfrak{B} \rightleftharpoons\left\langle a, b \mid\left\{\tau\left(K_{j} \alpha\right)=a \mid j=0,1,2, \ldots, m\right\}\right\rangle,
\end{aligned}
$$

где $K_{0}-$ пустое слово.

Доказательство очевидно.

Обозначим

$$
\mathscr{A}_{0} \rightleftharpoons\{a, b\}, \quad \overline{\mathscr{A}_{0}} \rightleftharpoons\{c, d\}, \quad \mathscr{A}^{\prime} \rightleftharpoons\{a, b, c, d, e\} .
$$

ОПРЕДЕЛЕНИЕ. Буквы $c$ и $d$ будем назьвать двойниками букв $a$ и $b$, и обратно. Мы используем обозначения:

$$
\bar{a} \rightleftharpoons c, \quad \bar{b} \rightleftharpoons d, \quad \bar{c} \rightleftharpoons a, \quad \bar{d} \rightleftharpoons b .
$$

Для произвольного слова $X$ в алфавите $\mathscr{A}_{0} \cup \overline{\mathscr{A}_{0}}$ через $\bar{X}$ будем обозначать результат замены в слове $X$ каждой буквы $\eta$ ее двойником $\bar{\eta}$.

Заметим, что если $X-$ слово в алфавите $\mathscr{A}_{0}$, а $Y$ - слово в алфавите $\overline{\mathscr{A}_{0}}$, то $X Y=$ $Y X$ в полугруппе Ц. 
ОПРеДЕЛЕНИЕ. Если алфавит $\mathscr{A}$ содержится в $\mathscr{B}$, а $X-$ слово в алфавите $\mathscr{B}$, то через $\left[X^{\mathscr{A}}\right.$ будем обозначать проекцию слова $X$ на алфавит $\mathscr{A}$.

Обозначим

$$
S \rightleftharpoons \overline{\tau\left(\alpha K_{0} \alpha K_{1} \alpha \ldots K_{m} \alpha\right)} .
$$

ОСНовнаЯ ЛЕмма. Если $P, Q-$ - лова в алфавите $\mathscr{A}_{0}$, mo

$$
P=Q \text { в полугруппе } \mathfrak{B} \Longleftrightarrow S P=S Q \text { в полугруппе } \amalg .
$$

ДОКАЗАТЕЛЬСТВО. Импликацию $\Longrightarrow$ достаточно доказать для случая одного элементарного преобразования $P \rightarrow Q$, причем можно ограничиться случаем:

$$
P=U a V, \quad Q=U \tau\left(K_{t} \alpha\right) V .
$$

Легко проверяются следуюшие равенства в полугруппе Ц:

$$
\begin{aligned}
S P & =S_{1} \overline{\tau\left(\alpha K_{t} \alpha\right)} S_{2} U a V=S_{1} U \overline{\tau\left(\alpha K_{t} \alpha\right)} a S_{2} V \\
& =S_{1} U\left[\overline{\tau\left(\alpha K_{t}\right)}\right) \overline{\tau(\alpha)} a S_{2} V=S_{1} U\left[\overline{\tau\left(\alpha K_{t}\right)}\right) c c a e S_{2} V \\
& =S_{1} U \overline{\tau\left(\alpha K_{t} \alpha\right)} a e S_{2} V=S_{1} U \overline{\tau(\alpha)} a\left(\overline{\tau\left(K_{t} \alpha\right)}\right] e S_{2} V \\
& =S_{1} U c c a e\left(\overline{\tau\left(K_{t} \alpha\right)}\right]\left(\tau\left(K_{t} \alpha\right)\right] S_{2} V=S_{1} U c c a\left(\overline{\tau\left(K_{t} \alpha\right)}\right]\left(\tau\left(K_{t} \alpha\right)\right] S_{2} V \\
& \left.=S_{1} c c \overline{\left(\overline{\tau\left(K_{t} \alpha\right)}\right]} S_{2} U a\left(\tau\left(K_{t} \alpha\right)\right] V=S_{1} \overline{\left(\tau\left(\alpha K_{t} \alpha\right)\right.}\right] S_{2} U \tau\left(K_{t} \alpha\right) V=S Q .
\end{aligned}
$$

Тем самым импликация $\Longrightarrow$ доказана.

Для доказательства импликации енам понадобятся следующие ниже леммы 2.4-2.6. Сначала индукциейпо длине слова $X$ в алфавите $\mathscr{A}^{\prime}$ определим функцию $\left[X^{\sim}\right.$ :

$$
\left[\Lambda^{\sim} \rightleftharpoons \Lambda, \quad\left[P \gamma ^ { \sim } \rightleftharpoons \left[P ^ { \sim } \left[\gamma^{\mathscr{A}_{0}}\right.\right.\right.\right.
$$

при $\gamma \in \mathscr{A}_{0} \cup \overline{\mathscr{A}_{0}}$ и

$$
\left[P e ^ { \sim } \rightleftharpoons \left[P^{\sim} \overline{\left[P^{\overline{\mathscr{A}_{0}}}\right.} .\right.\right.
$$

Очевидно, значениями функции [ $X \sim$ являются слова в алфавите $\mathscr{A}_{0}$. Если $P$ - слово в алфавите $\mathscr{A}_{0} \cup \overline{\mathscr{A}_{0}}$, то $\left[P^{\sim}=\left[P^{\mathscr{A}_{0}}\right.\right.$.

Следуюшая лемма доказьвается правой индукцией по длине слова $Q$.

Лемма 2.4. Для любых слов $P$ и $Q$ в алфавите $\mathscr{A}^{\prime}$ выполняется равенство

$$
\left[P Q^{\sim}=\left[P ^ { \sim } \left[\left[P^{\overline{A_{0}}} Q^{\sim} .\right.\right.\right.\right.
$$

Лемма 2.5. Если слово Рсс является началом слова $S$, то в полугруппе $\mathfrak{B}$ выполнено равенство а $\overline{P c c}=a$. 
Доказательство. Пусть слово $P c c$ есть начало $S$. Тогда слово $\overline{P c c}$ есть начало $\bar{S}$, т.е. начало слова $\tau\left(\alpha K_{0} \alpha K_{1} \alpha \ldots \alpha K_{m} \alpha\right)$. Так как слово $\overline{P c c}$ кончается словом $a a=\tau(\alpha)$ и буква $\alpha$ не входит в слова $K_{j}$, то при некотором $j \leqslant m$ имеем

$$
\overline{P c c}=\tau\left(\alpha K_{0} \alpha K_{1} \alpha \ldots \alpha K_{j} \alpha\right) \text {. }
$$

Используя соотношения полугруппы $\mathfrak{B}$, получим $\overline{P c c}=a$, и, следовательно, $a \overline{P c c}=$ $a a=a$.

Лемма 2.6. Если $P$ - слово в алфавите $\mathscr{A}_{0}$ и $S P=Q$ в полугруппе $\amalg$, то $\left[Q^{\sim}=P\right.$ в noлyгpynne $\mathfrak{B}$.

ДокАЗАТЕЛЬСтво. Пусть $P$ - слово в алфавите $\mathscr{A}_{0}$ и дана последовательность элементарных преобразований полугрупшы $\mathfrak{B}$

$$
S P \mp Q_{0} \rightarrow Q_{1} \rightarrow \cdots \rightarrow Q_{k} \rightarrow Q_{k+1}=Q .
$$

Имеем $\left[S P^{\sim}=P\right.$ и $\left[S P^{\overline{A_{0}}} \mp S\right.$. Легко видеть, что для любого слова $Q_{j}$ из последовательности (10) также вьполняется равенство $\left[Q_{j}^{\overline{\mathcal{A}_{0}}}\right.$ 픈. $S$.

Допустим, что искомое утверждение доказано для $Q_{k}$. Нам достаточно доказать, что при любом $k\left[Q_{k} \sim=\left[Q_{k+1} \sim\right.\right.$ в полугруппе $\mathfrak{B}$.

Рассмотрим следующие три случая.

I. $Q_{k}=U \gamma \delta V, Q_{k+1}=U \delta \gamma V\left(\gamma \in \overline{\mathscr{A}_{0}}, \delta \in \mathscr{A}_{0}\right)$.

II. $Q_{k}=U \gamma e V, Q_{k+1}=U e \gamma \bar{\gamma} V\left(\gamma \in \overline{\mathscr{A}_{0}}\right)$.

III. $Q_{k}=U c c a V, Q_{k+1}=U c c a e V$.

Случай I. $Q_{k}=U \gamma \delta V, Q_{k+1}=U \delta \gamma V\left(\gamma \in \overline{\mathscr{A}_{0}}, \delta \in \mathscr{A}_{0}\right)$.

По лемме 2.4 получаем

$$
\begin{aligned}
& {\left[Q_{k} \sim=\left[U \gamma \delta V^{\sim}=\left[U \gamma \delta ^ { \sim } \left[\left[U \gamma \delta^{\overline{\mathscr{A}_{0}}} V^{\sim}=\left[U ^ { \sim } \delta \left[\left[U^{\overline{A_{0}}} \gamma V^{\sim},\right.\right.\right.\right.\right.\right.\right.\right.} \\
& {\left[Q _ { k + 1 } \sim { } _ { \mathbb { I } } \left[U \delta \gamma V^{\sim}=\left[U \delta \gamma ^ { \sim } \left[\left[U \delta \gamma^{\overline{\mathcal{A}_{0}}} V^{\sim}=\left[U ^ { \sim } \delta \left[\left[U^{\overline{\mathcal{A}_{0}}} \gamma V^{\sim}=\left[Q_{k} \sim\right. \text {. }\right.\right.\right.\right.\right.\right.\right.\right.}
\end{aligned}
$$

Случай II. В этом случае вновь воспользуемся леммой 2.4 и определением функции $\left[X^{\sim}\right.$ :

$$
\begin{aligned}
& {\left[Q_{k} \sim=\left[U \gamma e V^{\sim}=\left[U \gamma e ^ { \sim } \left[\left[U \gamma^{\overline{\mathscr{A}_{0}}} V^{\sim}=\left[U ^ { \sim } \overline { [ U \gamma ^ { \overline { \mathscr { A } _ { 0 } } } } \left[\left[U \gamma^{\overline{\mathscr{A}_{0}}} V^{\sim},\right.\right.\right.\right.\right.\right.\right.\right.} \\
& {\left[Q _ { k + 1 } \sim \mathbb { } \left[\operatorname{Ue\gamma } \bar{\gamma} V^{\sim}=\left[U e \gamma \overline { \gamma } ^ { \sim } \left[\left[U \gamma^{\overline{\mathcal{A}_{0}}} V^{\sim}\right.\right.\right.\right.\right.} \\
& =\left[U e ^ { \sim } \overline { \gamma } \left[\left[U \gamma^{\overline{\mathscr{A}_{0}}} V^{\sim}=\left[U ^ { \sim } \overline { [ U ^ { \overline { \mathscr { A } _ { 0 } } } } \overline { \gamma } \left[\left[U \gamma^{\overline{\mathscr{A}_{0}}} V^{\sim}=\left[Q_{k} \sim\right. \text {. }\right.\right.\right.\right.\right.\right.
\end{aligned}
$$

Случай III. Имеем

$$
\begin{aligned}
{\left[Q_{k} \sim\right.} & =\left[U c c a V^{\sim}=\left[U c c a ^ { \sim } \left[\left[U^{\overline{\mathscr{A}_{0}}} c c V^{\sim}=\left[U ^ { \sim } a \left[\left[U^{\overline{A_{0}}} c c V^{\sim},\right.\right.\right.\right.\right.\right.\right. \\
{\left[Q_{k+1} \sim\right.} & =\left[U c c a e V^{\sim}=\left[U c c a e ^ { \sim } \left[\left[U^{\overline{\mathscr{A}_{0}}} c c V^{\sim}\right.\right.\right.\right. \\
& =\left[U c c a ^ { \sim } \overline { [ U c c ^ { \overline { \mathscr { A } _ { 0 } } } } \left[\left[U^{\overline{\mathcal{A}_{0}}} c c V^{\sim}=\left[U ^ { \sim } \overline { a } \overline { U ^ { \overline { A _ { 0 } } } c c } \left[\left[U^{\overline{\mathscr{A}_{0}}} c c V^{\sim} .\right.\right.\right.\right.\right.\right.
\end{aligned}
$$


Нам остается доказать, что в полугруппе $\mathfrak{B}$ вьполняется равенство

$$
a=a \overline{U^{\overline{A_{0}}} c c}
$$

Оно вьполнено в силу леммы 2.5 , так как слово $\left[U^{\overline{\mathcal{A}_{0}}} c c\right.$ является началом слова $\left[Q_{k}^{\overline{\mathcal{A}_{0}}}\right.$, а значит, и началом $S$. Лемма 2.6 доказана.

Теперь для доказательства импликации $\Longleftarrow$ в утверждении основной леммы нам достаточно воспользоваться леммой 2.6 , согласно которой мы получим

$$
P=\left[S Q^{\sim}=Q .\right.
$$

Возьмем в качестве полугруппы $G$ конечно определенную группу, имеющую неразрешимую проблему равенства (см. теорему 6.5). Разумеется, при этом в множестве определяюших соотношений $\left\{K_{j}=1 \mid j=1, \ldots, m\right\}$ должны быть указаны и все тривиальные соотношения $b_{s} b_{s}^{-1}=1, b_{s}^{-1} b_{s}=1$ группы $G$, которые обычно опускаются при задании групшы. Используя лемму 2.3 и основную лемму, получим, что для произвольного слова $P$ в алфавите образуюших полугруппы $G$ имеет место эквивалентность

$$
P=1 \text { в } G \Longleftrightarrow \tau(P)=a \text { в } \mathfrak{B} \Longleftrightarrow S \tau(P)=S a \text { в ц. }
$$

Следовательно, в полугруппе Ц неразрешима проблема распознавания равенства произвольного слова $X$ фиксированному слову $S a$. Теорема 2.2 Г. С. Цейтина доказана.

Заметим, что определяюшие соотношения полугруппы Ц не связаны с полугруппой $G$, а основная лемма означает, что равенство слов $P=Q$ в любой полугруппе $G$ можно имитировать в Ц посредством указанного вьше кодирования $\tau$ и умножения слева на фиксированное слово $S$, в котором закодирована система определяюших соотношений полугруппы $G$.

Полугруппа Г. С. Цейтина неоднократно использовалась при построении других простых примеров алгебраических систем с неразрешимой проблемой равенства.

В 1966 году Г. С. Маканин в работе [52] указал вложение полугруппы Г. С. Цейтина в полугруппу, заданную следуюшими 5 определяюшими соотношениями:

$$
\begin{aligned}
g g f f=f f g g, \quad f g g g f f & =g f f f g g, \quad f f g g f f f f g g=f f g g f f f f g g e, \\
e g g f f & =f f e, \quad \text { efgggff }=g f f e,
\end{aligned}
$$

которая, очевидно, также имеет неразрешимую проблему равенства слов.

В том же году Ю.В. Матиясевич построил общую конструкцию вложения произвольной конечно определенной полугруппы в полугруппу с 5 определяюшими соотношениями. Вскоре он усилил этот результат [61], построив примеры полугрупп c 3 определяюшими соотношениями и неразрешимой проблемой равенства. Доказательство этого результата приводится ниже.

Отправляясь от построенной при доказательстве теоремы 2.1 полугруппы $S\left(T_{0}\right)$, имеюшей неразрешимую проблему равенства слов, в задании которой ни одно определяющее слово не является пустым, легко построить полугруппу $\Pi_{1}$ с неразрешимой проблемой равенства слов, имеюшую задание, в котором все соотношения имеют вид $c=a b$. Для этого достаточно последовательно добавлять к заданиям, содержащим 
какое-то "длинное" соотношение вида $c d E=H$ (или $H=c d E$ ), новую букву $b$ и новое соотношения вида $b=c d$, а это "длинное" соотношение заменить на $b E=H$ $(H=b E)$.

Пусть полугруппа

$$
\Pi_{1}=\left\langle a_{1}, \ldots, a_{n} \mid A_{1}=B_{1}, \ldots, A_{m}=B_{m}\right\rangle,
$$

где $\left|A_{j}\right|=1,\left|B_{j}\right|=2$, имеет неразрешимую проблему распознавания равенства некоторому фиксированному слову $Q_{0}$.

При этом можно считать, что число $m$ определяюших соотношений полугруппы П 1 имеет вид $m=2^{k}$ и $m>n+3$.

По полугруппе $\Pi_{1}$ сначала построим некоторую полугрупп $\Pi_{2}$ с тремя образуюшими элементами и пятью определяюшими соотношениями, имеюшую неразрешимую проблему равенства слов. Для этого определим преобразование $\psi$, полагая

$$
\psi\left(a_{i}\right) \rightleftharpoons b^{2} c^{i} b c^{m-i-3} \quad(i=1,2, \ldots, n), \quad \psi(X Y) \rightleftharpoons \psi(X) \psi(Y)
$$

Заметим, что в слово $\psi(X)$ не входит $b^{3}$, так как $m>n+3$.

Пусть

$$
\begin{aligned}
& \psi\left(A_{j}\right)=p_{j 1} p_{j 2} \ldots p_{j m} \\
& \psi\left(B_{j}\right)=q_{j 1} q_{j 2} \ldots q_{j 2 m} .
\end{aligned}
$$

Положим

$$
\begin{aligned}
M & \rightleftharpoons p_{11} p_{21} \ldots p_{m 1} p_{12} p_{22} \ldots p_{m 2} \ldots p_{1 m} p_{2 m} \ldots p_{m m} \\
N & \rightleftharpoons q_{1{ }_{1}} q_{21} \ldots q_{m 1} q_{12} q_{22} \ldots q_{m 2} \ldots q_{12 m} q_{22} \ldots q_{m 2 m}
\end{aligned}
$$

Пусть

$$
\Pi_{2} \rightleftharpoons\langle b, c, d \mid b d=d b b, b d=d c b, c d=d b c, c d=d c c, M=N\rangle .
$$

Следующие две леммы очевидны.

ЛЕмма 2.7. Если $X$ - произвольное слово в алфавите $\{b, c\}$ u|X|=2 $2^{r}-1$, где $r>0$, mo $b d^{r}=d^{r} X b u c d^{r}=d^{r} X c$ в nолугрупnе $\Pi_{2}$.

ЛЕмма 2.8. Если $U-$ слово в алфавите $\{b, c\}$ длинъ $|U|=m-j$, mо $d^{k} U M=$ $\psi\left(A_{j}\right) d^{k} V u d^{k} U N=\psi\left(B_{j}\right) d^{k} V$, əдe $V=c^{|U|}$.

Если $E-$ любое слово из букв с и $b$, то

$$
\psi\left(A_{j}\right) E c d^{k}=d^{k} U M D \quad u \quad \psi\left(B_{j}\right) E c d^{k}=d^{k} U N D
$$

где $U-$ слово длиньи $m-j u d^{k} c^{m-j} D=E c d^{k}$.

ЛЕМма 2.9. Если $X=Y$ в nолугрупnе $\Pi_{1}$, mo $\psi(X) c d^{k}=\psi(Y) c d^{k}$ в $\Pi_{2}$. 
ДокаЗАТЕЛьство. Достаточно рассмотреть случай, когда переход $X \rightarrow Y$ есть элементарное преобразование в $\Pi_{1}$.

Пусть, например, $X=R A_{j} Q, Y=R B_{j} Q$. Тогда, используя леммы 2.7 и 2.8 , получаем в полугруппе $\Pi_{2}$ равенства

$$
\begin{aligned}
\psi(X) c d^{k} & =\psi(R) \psi\left(A_{j}\right) \psi(Q) c d^{k}=\psi(R) d^{k} U M D \\
& =\psi(R) d^{k} U N D=\psi(R) \psi\left(B_{j}\right) \psi(Q) c d^{k}=\psi(Y) c d^{k} .
\end{aligned}
$$

Лемма 2.9 доказана.

Лемма 2.10. Если $\psi(X) c d^{k}=\psi(Y) c d^{k}$ в полугруппе $\Pi_{2}$, әде $X, Y-$ слова в aлфавите полугруппы $\Pi_{1}$, то $X=Y$ в полугруппе $\Pi_{1}$.

ДокаЗАтЕльство. Пусть $\psi(X) c d^{k}=\psi(Y) c d^{k}$ в полугруппе $\Pi_{2}$. Рассмотрим последовательность элементарных преобразований

$$
\psi(X) c d^{k} \mp Z_{1} \rightarrow Z_{2} \rightarrow \cdots \rightarrow Z_{t}=\psi(Y) c d^{k} .
$$

В каждое слово $Z_{i}$ буква $d$ входит $k$ раз.

Обозначим через $\overline{Z_{i}}$ слово, полученное из слова $Z_{i}$ сдвигом всех вхождений буквы $d$ до предела вправо на основе первых четырех определяюших соотношений полугруппы $\Pi_{2}$. Индукцией по $i$ докажем, что для каждого $i$ существует такое слово $X_{i}$, равное $X$ в полугрупше $\Pi_{1}$, что $\overline{Z_{i}}=\psi\left(X_{i}\right) c d^{k}$.

В качестве $X_{1}$ возьмем $X$. Допустим, что уже установлено $\overline{Z_{i}} \equiv \psi\left(X_{i}\right) c d^{k}$, где $X=X_{i}$ в полугруппе $\Pi_{1}$, и сделаем то же самое для слова $\overline{Z_{i+1}}$.

Если в переходе $Z_{i} \rightarrow Z_{i+1}$ не использовалось определяюшее соотношение $M=N$, то $\overline{Z_{i+1}} \equiv \overline{Z_{i}}$ и в качестве искомого слова $X_{i+1}$ можно взять $X_{i}$.

Пусть переход $Z_{i} \rightarrow Z_{i+1}$ совершен с использованием соотношения $M=N$. Можно считать, что это левое преобразование, т.е. при некоторых $u$ и $v$

$$
Z_{i}=u M v, \quad Z_{i+1}=u N v .
$$

Так как $2 m=2^{k+1}$ и в слове $\psi\left(X_{i}\right)$ нет вхождений $b^{3}$, то справедливо следующее утверждение.

ЗАмЕчАниЕ. Если слово $Z$ получено из слова вида $\psi(X) c d^{k}$ с помошью только первых четырех соотношений полугруппы $\Pi_{2}$, то в слове $Z$ все $k$ вхождений буквы $d$ расположены левее любого подслова вида $b^{2 m}$.

Поэтому в слове $Z_{i}$ все $k$ букв $d$ должны быть левее подслова $M$. Максимально приблизив все эти буквы $d$ слева к подслову $M$, мы можем считать, что $u=u_{1} d^{k} u_{2}$, где $\left|u_{2}\right|<m$, так как по индуктивному предположению $Z_{i}$ получается из $\overline{Z_{i}}$ одними сдвигами букв $d$. При этом $u_{1}$ есть начало слова $\psi\left(X_{i}\right)$, так как слово $\overline{Z_{i}}=\psi\left(X_{i}\right) c d^{k}$ можно считать результатом сдвига вправо всех букв $d$ в слове $u_{1} d^{k} u_{2} M v$.

Пусть $\left|u_{2}\right|=m-j$. Очевидно, при некоторых $M_{1}$ и $N_{1}$ имеем $M=M_{1} c^{\left|u_{2}\right|}$ и $N=$ $N_{1} c^{\left|u_{2}\right|}$. Тогда $d^{k} u_{2} M_{1}=\psi\left(A_{j}\right) d^{k}$ и $d^{k} u_{2} N_{1}=\psi\left(B_{j}\right) d^{k}$. Так как $\overline{Z_{i}}=\psi\left(X_{i}\right) c d^{k}$, то $\overline{Z_{i}}$ есть результат сдвига вправо всех букв $d$ в слове $Z_{i}=u_{1} \psi\left(A_{j}\right) d^{k} c^{\left|u_{2}\right|} v$, т.е. имеем $d^{k} c^{\left|u_{2}\right|} v=Z d^{k}$ при некотором $Z$. Следовательно, имеем $\psi\left(X_{i}\right) c d^{k}=u_{1} \psi\left(A_{j}\right) Z d^{k}$, т.е. $\psi\left(X_{i}\right) c=u_{1} \psi\left(A_{j}\right) Z$. Учитьвая, что никакие два образа $\psi\left(a_{i}\right)$ не пересекаются, 
отсюда получаем, что $u_{1} \approx \psi(P)$ и $Z \approx \psi(Q) c$ при некоторых $P$ и $Q$. Следовательно, $X_{i} \mp P A_{j} Q$.

Таким образом, в $\Pi_{2}$ получаем равенства

$$
\begin{aligned}
Z_{i+1} & =u N v=\psi(P) d^{k} u_{2} N_{1} c^{\left|u_{2}\right|} v=\psi(P) \psi\left(B_{j}\right) d^{k} c^{\left|u_{2}\right|} v \\
& =\psi(P) \psi\left(B_{j}\right) Z d^{k}=\psi(P) \psi\left(B_{j}\right) \psi(Q) c d^{k}=\psi\left(P B_{j} Q\right) c d^{k} .
\end{aligned}
$$

Это значит, что мы можем взять в качестве искомого $X_{i+1}$ слово $P B_{j} Q$, которое равно $X$ в $\Pi_{1}$. Лемма 2.10 доказана.

Из лемм 2.9 и 2.10 следует, что в полугрупше $\Pi_{2}$ неразрешима проблема распознавания равенства слов вида $\psi(X) c d^{k}$ фиксированному слову $\psi\left(Q_{0}\right) c d^{k}$.

Рассмотрим следующее отображение $\tau$ :

$$
\begin{gathered}
\tau(b) \rightleftharpoons \beta, \quad \tau(c) \rightleftharpoons \beta \alpha, \quad \tau(d) \rightleftharpoons \alpha \alpha, \\
\tau(1) \rightleftharpoons 1, \quad \tau(X Y) \rightleftharpoons \tau(X) \tau(Y) .
\end{gathered}
$$

Обозначим через $\Pi_{3}$ полугруппу, заданную образующими $\alpha, \beta$ и следующими определяющими соотношениями:

$$
\begin{array}{ll}
\text { (i) } \alpha \alpha \beta \alpha \beta=\beta \alpha \alpha, & \text { (ii) } \alpha \alpha \beta \beta=\beta \alpha \alpha, \\
\text { (iii) } \alpha \alpha \beta \alpha \beta \alpha=\beta \alpha \alpha \alpha, & \text { (iv) } \alpha \alpha \beta \beta \alpha=\beta \alpha \alpha \alpha,
\end{array}
$$

(v) $\tau(M)=\tau(N)$.

Следующие две леммы тривиальны.

Лемма 2.11. Eсли $X=Y$ в noлугрynпе $\Pi_{2}$, mo $\tau(X)=\tau(Y)$ в noлугрynnе $\Pi_{3}$.

ЛЕмма 2.12. Если $\tau(X)=P \beta E$, то при некоторых $X_{1}$ и $X_{2}$ выполняются равенства

$$
X=X_{1} X_{2}, \quad P=\tau\left(X_{1}\right), \quad \beta E=\tau\left(X_{2}\right) .
$$

ЛЕмма 2.13. $Е с л и \tau(X)=\tau(Y)$, mo $X \mp Y$.

ДокАЗАТЕЛЬСТво. Если слово $\tau(X)$ не имеет вхождений буквы $\beta$ или $\tau(X)=\beta Z$, где $\beta$ не входит в $Z$, то равенство $X=Y$ очевидно.

Если же $\tau(X)=P \beta E$, где слово $P$ непусто и может содержать $\beta$ только в начале, то по предыдушей лемме имеем $X \mp X_{1} X_{2}$ и при этом $P=\tau\left(X_{1}\right)$ и $\beta E=\tau\left(X_{2}\right)$. Далее, однозначность $X_{1}$ мы имеем по доказанному, а однозначность $X_{2}$ получаем по индуктивному предположению.

ЛЕмма 2.14. Eсли $\psi(X) c d^{k}=Z$ в полугруппе $\Pi_{2}$ u переход $\tau(Z) \rightarrow Y$ является әлементарным преобразованием в $\Pi_{3}$, то $Y=\tau\left(Z_{1}\right)$ для некоторого слова $Z_{1}$, причем $Z \rightarrow Z_{1}$ является әлементарным преобразованием в $\Pi_{2}$. 
ДокАЗАТЕльство. Пусть $\tau(X)=P E Q$ и $Y=P D Q$ для некоторого определяющего соотношения $E=D$ полугруппы П 3 . Так как слово $E$ начинается либо с $\beta$, либо с $\alpha \alpha \beta$, то по предыдущей лемме найдем такие слова $X_{1}$ и $X_{2}$, что

$$
X=X_{1} X_{2}, \quad P=\tau\left(X_{1}\right), \quad E Q=\tau\left(X_{2}\right) .
$$

Пусть $S$ есть максимальное начало слова $X_{2}$ такое, что $\tau(S)$ является началом слова $E$, т.е. $E \mp \tau(S) E_{1}$ при некотором $E_{1}$. Тогда $X_{2}=S X_{3}$ и $E_{1} Q=\tau\left(X_{3}\right)$ при некотором $X_{3}$.

Если слово $E_{1}$ пусто, то $E=\tau(S), Q=\tau\left(X_{3}\right)$ и для некоторого определяющего соотношения $S=L$ полугруппы $\Pi_{2}$ имеем $D=\tau(L)$ и $Y=\tau\left(X_{1} L X_{3}\right)$. Тогда слово $X_{1} L X_{3}$ можно взять в качестве искомого слова $Z$.

Пусть $E_{1}$ непусто. Тогда слово $X_{3}$ начинается с буквы $c$ или $d$, образ которой разделился между $E$ и $Q$. При этом $\left|E_{1}\right|=1$ и слово $Q$ начинается с буквы $\alpha$, т.е. $Q=\alpha Q_{1}$ при некотором $Q_{1}$. Докажем, что $E \alpha=D \alpha$ также является определяющим соотношением полугруппы $\Pi_{3}$.

1. Если $E_{1}=\beta$, то равенство $E=D$ является определяюшим соотношением (i) или (ii), так как слово $E$ кончается на $\beta$, а слова $\tau(M)$ и $\tau(N)$ кончаются на $\beta \alpha$. В этом случае мы можем воспользоваться вместо определяюшего соотношения $E=D$ определяюшим соотношением $E \alpha=D \alpha$ и найдем искомое слово $Z$, как в случае пустого $E_{1}$.

2. Пусть $E_{1} \approx \alpha$. Тогда $E \mp \tau(S) \alpha$ и

$$
X_{3}=d X_{4}, \quad Q_{1} \mp \tau\left(X_{4}\right)
$$

при некотором $X_{4}$. Значит, буква $d$ входит в слово $X$ правее слова $S$. Если бы при этом слово $E$ совпадало с одним из слов $\tau(M)$ или $\tau(N)$, то в слово $S$ входило бы слово $b^{2 m}$. Но, как мы убедились при доказательстве леммы 2.10 , в слове $X$, равном в полугруппе $\Pi_{2}$ слову $\psi\left(Z_{1}\right) c d^{k}$, левее буквы $d$ не может входить слово $b^{2 m}$. Следовательно, определяюшее соотношение $E=D$ есть одно из определяюших соотношений (i), (ii), (iii) или (iv).

2.1. Если при этом $D=D_{1} \alpha$, то $E=D$ есть одно из соотношений (iii) или (iv). Тогда $D_{1}=\tau(S)$ есть одно из соотношений (i) или (ii). Легко видеть, что тогда $D_{1}=$ $\tau\left(L_{1}\right)$ для некоторого $L_{1}$ и равенство $S=L_{1}$ также есть определяюше соотношение полугруппы $\Pi_{2}$. В этом случае полагаем $Z \rightleftharpoons X_{1} L_{1} X_{3}$.

2.2 . Если слово $D$ кончается на $\beta$, то равенство $E=D$ есть одно из определяющих соотношений (i) или (ii). Тогда вместо него мы можем использовать определяюшее соотношение $E \alpha=D \alpha$, где $D \alpha=\tau(L)$ для некоторого $L$. В этом случае можно полагать $Z \rightleftharpoons X_{1} L X_{4}$, как в случае пустого $E_{1}$.

Это завершает доказательство леммы 2.14.

Из лемм 2.9, 2.10, 2.11 и 2.14 непосредственно следует

ЛЕмма 2.15. Для любых двух слов $X$ и $Y$ в алфавите полугруппь $\Pi_{1}$ имеет место әквивалентность

$$
X=Y \text { в noлyгpynne } \Pi_{2} \Longleftrightarrow \tau\left(\psi(X) c d^{k}\right)=\tau\left(\psi(Y) c d^{k}\right) \text { в noлyгpynne } \Pi_{3} .
$$

Из лемм 2.7 и 2.15 следует, что проблема распознавания равенства слов в полугруппе $\Pi_{3}$ неразрешима.

Так как определяюшие соотношения (iii) и (iv) вытекают из соотношений (i) и (ii), то мы доказали следующую теорему. 
TEOPEмA 2.3. B полугруппе $\Pi_{3}$, заданной порождающими $\alpha, \beta$ и определяющими соотношениями

$$
\tau(M)=\tau(N), \quad \alpha \alpha \beta \beta=\beta \alpha \alpha, \quad \beta \alpha \alpha=\alpha \alpha \beta \alpha \beta,
$$

неразрешима проблема распознавания равенства фиксированному слову $\tau\left(\psi\left(Q_{0}\right) c d^{k}\right)$ для слов вида $\tau\left(\psi(X) c d^{k}\right)$, где $X$ - произвольное слово в алфавите образующих полугруппь $\Pi_{1}$.

\section{3. Некоторые классы полугрупп с разрешимой проблемой равенства}

Укажем некоторые классы полугрупп, для которых сушествуют простые алгоритмы распознавания равенства слов.

Сначала заметим, что для любой конечно определенной полугруппы П множество всех слов, равных данному слову $Q$, алгоритмически перечислимо. В самом деле, определив $W_{k}(Q)$ как множество всех слов, которые могут быть получены из $Q$ посредством не более чем $k$ последовательных элементарных преобразований (полагая при этом $\left.W_{0}(Q) \rightleftharpoons\{Q\}\right)$, мы можем вычислять $W_{k+1}(Q)$ из множества $W_{k}(Q)$, добавив к нему все слова, полученные из слов этого множества одним элементарньм преобразованием.

Легко видеть, что проблема равенства решается очень просто для всех полугрупп, удовлетворяюших условию однородности определяюших соотношений, т.е. при $\left|A_{i}\right|=\left|B_{i}\right|$ для всех соотношений $A_{i}=B_{i}$. Пусть нам нужно проверить равенство в такой полугруппе $X=Q$. Так как в любой такой полугруппе все слова, равные данному слову $Q$, имеют ту же длину, то все указанные вьше множества $W_{k}(Q)$ будут содержать не более чем $n^{|Q|}$ слов, где $n$ - число порождающих. Поэтому нам остается найти такое $k$, при котором $W_{k+1}(Q)=W_{k}(Q)$, и проверить, есть ли в $W_{k}(Q)$ слово $X$.

Проблема равенства имеет весьма простое решение также и для свободных групп $F_{n}$, так как каждое слово с помощью сокращений легко приводится к несократимому виду, а два несократимых слова равны в $F_{n}$ тогда и только тогда, когда они совпадают графически.

Легко также указать алгоритм для решения проблемы равенства в полугруппах, заданных конечным числом определяющих соотношений, при условии что нам известна конечность рассматриваемой полугрупшы. Пусть конечная полугруппа П задана определяюшими соотношениями и в ней нужно проверить равенство $X=Q$. Для этого мы будем применять параллельно два алгоритма $\mathfrak{A}$ и $\mathfrak{B}$. Алгоритм $\mathfrak{A}$ перечисляет все слова $U$, равные слову $Q$ в полугруппе $\Pi$, как указано вьше, и устанавливает равенство $X=Q$ в случае, если оно имеет место в $П$.

Алгоритм $\mathfrak{B}$ перебирает всевозможные гомоморфизмы $\varphi: \Pi \rightarrow S$ полугруппы П в конечные полугруппы $S$, задаваемые таблицей Кэли, т.е. такие отображения $\varphi$, при которых образы $\varphi(A)$ и $\varphi(B)$ совпадают для каждого определяюшего соотношения $A=B$ полугрупшы П. Для каждого такого гомоморфизма наш алгоритм проверяет, совпадают ли образы данных слов $\varphi(X)$ и $\varphi(Q)$ в полугрупше $S$, и останавливается, найдя такой гомоморфизм, при котором эти образы не совпадают.

Очевидно, если слова $X$ и $Q$ не равны в конечной полугруппе П, то алгоритм $\mathfrak{B}$ через конечное число шагов установит это. 
По сушеству алгоритмы $\mathfrak{A}$ и $\mathfrak{B}$ дают решение проблемы равенства слов для всех финитно аппроксимируемых конечно определенных полугрупп (и в том числе для финитно аппроксимируемых групп). Полугруппа (группа) П называется финитно аnпроксимируемой, если для любых двух ее неравных слов $A$ и $B$ сушествует такой ее гомоморфизм $\varphi: \Pi \rightarrow S$ в конечную полугруппу (соответственно, в конечную группу) $S$, что $\varphi(A)$ не совпадает с $\varphi(B)$.

Отметим также результат $\Phi$. Холла [38] о финитной аппроксимируемости конечно порожденных разрешимых групп ступени 2.

Вопрос о разрешимости проблемы равенства слов в полугруппах с условием малого налегания определяюших слов рассматривался В. А. Осиповой в работе [80]. В этой работе доказана разрешимость проблемы равенства слов для полугрупп, заданных определяющими соотношениями вида

$$
A_{i}=B_{i} \quad(i=1,2, \ldots, m)
$$

где все слова $A_{i}$ и $B_{i}$ не пусты и имеют меру налегания строго меньше $1 / 2$. Там же был построен пример полугруппы с неразрешимой проблемой равенства слов и мерой налегания, равной $1 / 2$.

Пусть полугруппа П задана образующими

$$
a_{1}, \ldots, a_{n}
$$

и определяюшими соотношениями

$$
A_{i}=B_{i} \quad(i=1,2, \ldots, k)
$$

причем все слова $A_{i}$ и $B_{i}$ непустые.

Через $M$ обозначим множество всех определяющих слов $A_{i}$ и $B_{i}$ полугруппы П.

Начало (конец) $P$ слова $X$ будем назьвать правильнылм началом (правильным кониом) слова $X$, если $|P| \geqslant|X| / 2$.

ОПРЕДЕЛЕНИЕ. Будем говорить, что полугруппа П, заданная образующими (1) и определяюшими соотношениями (2), принадлежит классу $K_{1 / 2}$, если для любого представления слов $A$ из $M$ в виде $A \mp R P Q$ вьполняются условия:

a) если $P$ - правильное начало некоторого слова из $M$, то слово $R$ пусто,

б) если $P$ - правильньй конец некоторого слова из $M$, то слово $Q$ пусто.

Определение класса полугрупп $\overline{K_{1 / 2}}$ получается из определения класса полугрупп $K_{1 / 2}$ заменой в определении правильных начал и правильных концов нестрогих неравенств $|P| \geqslant|X| / 2$ на строгие $|P|>|X| / 2$. Ясно, что $K_{1 / 2} \subseteq \overline{K_{1 / 2}}$.

Tеорема 3.1. Если $\Pi \in K_{1 / 2}$, то каждое слово $X$ в алфавите полугруппьи П имеет лишь конечное число равных ему в П слов. Точнее, если $X$ и $Y$ - слова в алфавите П $и X=Y$ в полугруппе $\Pi$, то выполняется неравенство

$$
|Y| \leqslant|X| m^{|X|}, \quad \text { zдe } m \rightleftharpoons \max _{1 \leqslant i \leqslant k}\left\{\left|A_{i}\right|,\left|B_{i}\right|\right\} .
$$


СлЕДСТВИЕ 3.1.1. Существует алгоритм, решающий проблему равенства слов для всех полугрупп из класса $K_{1 / 2}$.

ОПРеДЕЛЕНИЕ. Пусть $\Pi \in K_{1 / 2}$. Разложение

$$
E=S_{1} S_{2} \ldots S_{t}
$$

слова $E$ в алфавите (1) назовем нормальнымм, если слова $S_{1}, S_{2}, \ldots, S_{t}$ удовлетворяют условиям:

1) Каждое $S_{i}$ входит в некоторое $C_{i} \in M$.

2) $S_{1}$ - правильное начало $C_{1}$, а $S_{t}$ - правильный конец $C_{t}$.

3) Для каждого $i<t$ либо $S_{i}-$ правильньй конец $C_{i}$, либо $S_{i+1}$ - правильное начало $C_{i+1}$.

Лемма 3.1. Если слово $P A Q$, где $A \in M$, имеет нормальное разложсение, состоящее из $t$ множителей, то можсно указать такое нормальное разложение слова PAQ из одним из сомножителей $S_{i}$.

ДокАЗАТЕЛЬСтво. Пусть $P A Q \mp R_{1} R_{2} \ldots R_{t}$ - нормальное разложение, где $C_{k}=u_{k} R_{k} v_{k}, C_{k} \in M(k=1, \ldots, t)$.

Рассмотрим первое $R_{i}$, которое пересекается с $A$, т.е. найдем такое $i \leqslant t$, что $P=$ $R_{1} R_{2} \ldots R_{i}^{\prime}, R_{i}=R_{i}^{\prime} A^{\prime}, A=A^{\prime} A^{\prime \prime}$, где $A^{\prime}$ непусто и одно из слов $A^{\prime \prime}$ и $R_{i+1}$ есть начало другого.

Рассмотрим два случая:

1) $\left.\left|A^{\prime}\right| \geqslant|A| / 2,2\right)\left|A^{\prime}\right|<|A| / 2$.

Случай 1). $A^{\prime}$ есть правильное начало слова $A$ и может входить в $C_{i}$ только как его начало. Следовательно, $R_{i} \mp A^{\prime}$. Если $A^{\prime \prime}$ пусто, то имеем $R_{i} \mp A$ и в качестве искомого разложения можно взять исходное.

Если слово $R_{i}$ является правильным концом, то оно есть конец слова $A$, т.е. мы опять имеем случай, когда $A^{\prime \prime}$ пусто. Следовательно, можно считать, что $R_{i+1}$ - правильное начало.

Так как $A^{\prime}$ непусто, то $R_{i+1}$ не может быть началом $A^{\prime \prime}$. Следовательно, $A^{\prime \prime}$ есть начало $R_{i+1}$, т.е. $R_{i+1}=A^{\prime \prime} R_{i+1}^{\prime \prime}$ при некотором непустом $R_{i+1}^{\prime \prime}$.

Наконец заметим, что если $R_{i+1}-$ правильный конец, то $R_{i+1}=C_{i+1}$ и слово $R_{i+1}^{\prime \prime}$ - также будет правильным концом слова $C_{i+1}$, так как в силу непустоты $A^{\prime}$ слово $A^{\prime \prime}$ не может быть правильньм началом слова $C_{i+1}$.

Теперь мы можем построить искомое нормальное разложение $S_{1} S_{2} \ldots S_{t}$ слова $P A Q$, полагая

$$
S_{1} \rightleftharpoons R_{1}, \ldots, S_{i-1} \rightleftharpoons R_{i-1}, S_{i} \rightleftharpoons A, S_{i+1} \rightleftharpoons R_{i+1}^{\prime \prime}, S_{i+2} \rightleftharpoons R_{i+2}, \ldots, S_{t} \rightleftharpoons R_{t} .
$$

Случай 2). $\left|A^{\prime}\right|<|A| / 2$, т.е. $A^{\prime \prime}$ - правильный конец слова $A$. Напомним, что одно из слов $A^{\prime \prime}$ и $R_{i+1}$ есть начало другого. Если бы $A^{\prime \prime}$ было началом $R_{i+1}$, то оно должно было бы быть концом соответствуюшего слова $C_{i+1}$ и мы имели бы $R_{i+1} \mp A^{\prime \prime}$. Следовательно, можно ограничиться случаем, когда $R_{i+1}$ есть начало $A^{\prime \prime}$, т.е. для некоторого слова $Z$ вьполняется равенство

$$
A^{\prime \prime}=R_{i+1} Z \text {. }
$$


Так как по условию $A^{\prime}$ непусто, то $R_{i+1}$ не является правильным началом. Тогда $R_{i}-$ правильный конец. Если бы при этом слово $R_{i}^{\prime}$ было пусто, то мы имели бы равенство $R_{i}=A^{\prime}$. Тогда условие б) из определения класса $K_{1 / 2}$ вместе с равенством $A \mp A^{\prime} A^{\prime \prime}$ влекло бы пустоту слова $A^{\prime \prime}$, что в рассматриваемом случае неверно. Поэтому слово $R_{i}^{\prime}$ непусто.

Заметим, что если при этом слово $R_{i}$ есть правильное начало, то мы имеем равенство $C_{i}=R_{i}=R_{i}^{\prime} A^{\prime}$. Отсюда следует, что слово $R_{i}^{\prime}$ есть правильное начало слова $C_{i}$, так как в силу непустоты $A^{\prime \prime}$ слово $A^{\prime}$ не может быть правильным концом $C_{i}$.

В случае, если слово $R_{i+1}$ - правильный конец, то слово $Z$ пусто. Тогда искомое разложение получаем в виде

$$
S_{1} \rightleftharpoons R_{1}, \ldots, S_{i-1} \rightleftharpoons R_{i-1}, S_{i} \rightleftharpoons R_{i}^{\prime}, S_{i+1} \rightleftharpoons A, S_{i+2} \rightleftharpoons R_{i+2}, \ldots, S_{t} \rightleftharpoons R_{t} .
$$

Остается рассмотреть случай, когда слово $R_{i+1}$ не является правильным концом. Тогда $R_{i+2}$ - правильное начало. В силу (3) слово $R_{i+2} Y$ не есть начало $Z$, так как по условию $A^{\prime}$ непусто.

Значит, $R_{i+2} \mp Z R_{i+2}^{\prime \prime}$ при некотором непустом $R_{i+2}^{\prime \prime}$. При этом имеем также равенство $A=A^{\prime} R_{i+1} Z$. Кроме того, заметим, что если $R_{i+2}$ является правильньм концом, то $R_{i+2} \mp C_{i+2}$, и тогда $R_{i+2}^{\prime \prime}$ также будет правильным концом слова $C_{i+2}$, так как $|Z|<\left|C_{i+2} / 2\right|$. Для завершения доказательства леммы 3.1 достаточно положить

$$
\begin{aligned}
S_{1} \rightleftharpoons R_{1}, \ldots, S_{i-1} \rightleftharpoons R_{i-1}, S_{i} \rightleftharpoons R_{i}^{\prime} \\
S_{i+1} \rightleftharpoons A, S_{i+2} \rightleftharpoons R_{i+2}^{\prime \prime}, S_{i+3} \rightleftharpoons R_{i+3}, \ldots, S_{t} \rightleftharpoons R_{t} .
\end{aligned}
$$

Из доказанной леммы 3.1 сразу следует

Лемма 3.2. Если слово $P A Q$ имеет нормальное разложсение из $t$ множителей, а переход $P A Q \rightarrow P B Q$ является әлементарным преобразованием полугруппь П, то слово РВQ также имеет нормальное разложсение из $t$ множителей.

Из леммы 3.2 следует

ЛЕмма 3.3. Если $V$ есть максимальное нормальное начало слова $V Q$, а переход $V \rightarrow V_{1}$ является әлементарным преобразованием полугруппы П, то слово $V_{1}$ есть максимальное нормальное начало слова $V_{1} Q$.

ЛЕмма 3.4. Если $V$ - максимальное нормальное начало слова $V Y$ и $V Y$ ш $P A Q$, где $A \in M_{\Pi}$, то либо $|V|<|P|$, либо $|V| \geqslant|P A|$.

ДоказАтельство. Очевидно, слова $V$ и $P$ не совпадают. Предположим, что $|P|<|V|<|P A|$. Тогда $V=P A^{\prime}, A=A^{\prime} A^{\prime \prime}, Y=A^{\prime \prime} Q$, причем слова $A^{\prime}$ и $A^{\prime \prime}$ непусты. Пусть $R_{1}, \ldots, R_{t}$ - нормальное разложение слова $V$. Достаточно доказать, что $A^{\prime \prime}$ - правильный конец. Если это не так, то слово $A^{\prime}$ - правильное начало. Из слов $R_{t}$ и $A^{\prime}$ одно является концом другого. Так как $A^{\prime \prime}$ непусто, то правильный конец $R_{t}$ не может быть концом $A^{\prime}$. Следовательно, $A^{\prime}$ есть конец $R_{t}$. Тогда $R_{t}=A^{\prime}=A$, так как $A^{\prime}$ - правильное начало. Но это противоречит условию непустоты слова $A^{\prime \prime}$. Это завершает доказательство леммы 3.4 . 
ЛЕмма 3.5. Если $X=Y$ в $\Pi$, mo

$$
|Y| \leqslant|X| m^{|X|}, \quad \text { əде } m \rightleftharpoons \max _{1 \leqslant i \leqslant k}\left\{\left|A_{i}\right|,\left|B_{i}\right|\right\}
$$

ДокАЗАТЕльСТво. Индукция по длине слова $X$. Так как по условию все определяющие слова полугрупшы П непустые, то можно считать, что $|X|>0$. Пусть $X \mp a W$, где $a$ - буква. Полагаем $l \rightleftharpoons|X|$. В силу индуктивного предположения любое слово $W_{j}$, равное слову $W$ в полугруппе П, имеет длину, не превосходящую $(l-1) m^{l-1}$.

Из всех последовательностей элементарных преобразований вида

$$
a W_{j} \mp F_{0} \rightarrow F_{1} \rightarrow \cdots \rightarrow F_{p} \mp Y
$$

для слов $W_{j}$, равных слову $W$ в полугруппе П, выберем последовательность наименьшей длины

$$
a W_{k}=E_{0} \rightarrow E_{1} \rightarrow \cdots \rightarrow E_{i} \rightarrow E_{i+1} \rightarrow \cdots \rightarrow E_{r} \mp Y
$$

Пусть $a W_{k}=V Z$, где $V$ - максимальное нормальное начало слова $a W_{k}$. Очевидно, первое элементарное преобразование кратчайшей последовательности (4) должно затронуть начальную букву $a$, т.е. оно имеет вид

$$
a W_{k}=A Q \rightarrow B Q=E_{1} .
$$

Следовательно, нормальное слово $V$ непусто.

Индукцией по $i$ докажем, что $E_{i}=V_{i} Z$, где $V_{i}$ - максимальное нормальное начало слова $E_{i}$, и существует последовательность

$$
a V^{\prime}=V_{0} \rightarrow V_{1} \rightarrow \cdots \rightarrow V_{r}
$$

содержашая ровно $r$ элементарных преобразований.

Индуктивньй шаг от $i$ к $i+1$ осушествляется следуюшим образом. Пусть элементарное преобразование $E_{i} \rightarrow E_{i+1}$ имеет вид $P A Q \rightarrow P B Q$, где $A, B \in M$. По лемме 3.4 либо $\left|V_{i}\right|<|P|$, либо $\left|V_{i}\right| \geqslant|P A|$.

Если при некотором $i$ имеет место $\left|V_{i}\right|<|P|$ (мы рассматриваем первое такое преобразование), то при некотором $P^{\prime}$ выполнены равенства:

$$
P=V_{i} P^{\prime}, \quad Z=P^{\prime} A Q,
$$

а преобразование $E_{i} \rightarrow E_{i+1}$ по существу происходит в $Z$. В таком случае имеем равенства $W_{k}=V^{\prime} Z=V^{\prime} P^{\prime} A Q=V^{\prime} P^{\prime} B Q$ в полугруппе П, и, следовательно, $W=V^{\prime} P^{\prime} B Q$ в полугруппе П. Поэтому, взяв вместо $W_{k}$ равное ему слово $V^{\prime} P^{\prime} B Q$, мы построили бы более короткую, чем (4), последовательность элементарных преобразований

$a V^{\prime} P^{\prime} B Q=V_{0} P^{\prime} B Q \rightarrow V_{1} P^{\prime} B Q \rightarrow \cdots \rightarrow V_{t} P^{\prime} B Q=E_{i+1} \rightarrow E_{i+2} \rightarrow \cdots \rightarrow F_{r}=Y$. 
Следовательно, во всех преобразованиях кратчайшей последовательности (4) должен иметь место случай $\left|V_{i}\right| \geqslant|P A|$. Тогда при каждом $i$ имеем $V_{i}=P A V_{i}^{\prime}, Q=V_{i}^{\prime} Z$, т.е. $E_{i+1} \mp P B V_{i}^{\prime} Z$ и переход $P A V_{i}^{\prime} \rightarrow P B V_{i}^{\prime}$ является элементарным преобразованием полугруппы П, причем по лемме 3.3 слово $P B V_{i}^{\prime}$ есть максимальное нормальное начало слова $P B V_{i}^{\prime} Z=E_{i+1}$, т.е. $V_{i+1}=P B V_{i}^{\prime} Z$.

Значит, $Y=V_{r} Z$ и $V=V_{r}$ в полугруппе П. По лемме 3.2 число множителей в нормальном разложении слова $V_{r}$ равно числу множителей в нормальном разложении слова $V$, а значит, не превосходит $|V|$. Это дает неравенства $\left|V_{r}\right| \leqslant m|V|$ и

$$
\begin{aligned}
|Y| & =\left|V_{r}\right|+|Z| \leqslant m\left[1+(l-1) m^{l-1}-|Z|\right]+|Z| \\
& =|Z|(1-m)+l m^{l}+m\left(1-m^{l-1}\right) \leqslant l m^{l} .
\end{aligned}
$$

Доказательство леммы 3.5 завершено.

Из доказанной леммы сразу следует теорема 3.1 .

В. А. Осипова также построила конечно определенную полугруппу из класса $\overline{K_{1 / 2}}$, которая имеет неразрешимую проблему равенства слов. В своей диссертации [81] она доказала, что приведенная выше полугрупша Г. С. Цейтина Ц с неразрешимой проблемой равенства слов вкладьвается в полугруппу, заданную следующими определяюшими соотношениями:

$$
\begin{gathered}
a c=c a, \quad a d=d a, \quad b c=c b, \quad b d=d b, \\
e=m n, \quad n c=f p, \quad m f=c q, \quad q p a=e, \\
e=m^{\prime} n^{\prime}, \quad n^{\prime} d=f^{\prime} p^{\prime}, \quad m^{\prime} f^{\prime}=d q^{\prime}, \quad q^{\prime} p^{\prime} b=e, \\
c c=\varepsilon \delta, \quad e=\eta \gamma, \quad \delta a \eta=\varphi \psi, \quad \psi \gamma=\chi c, \quad \varepsilon \varphi \chi=c a .
\end{gathered}
$$

При этом использовалась техника, изложенная в работе [10].

\section{4. Полугруппы с одним определяюшим соотношением}

Хотя проблема равенства для групп с одним определяюшим соотношением была решена В. Магнусом еше в 1932 году (см. теорему 7.6 ниже), в вопросе о разрешимости проблемы равенства для полугрупп с одним определяюшим соотношением долгое время не было сдвигов. Некоторые результаты по этой проблеме были получены С. И. Адяном в 60-х годах. Они изложены в монографиии [10]. Впоследствии эта работа была продолжена как самим автором, так и его учениками. Однако проблему равенства в обшем случае для полугрупп с одним определяюшим соотношением решить пока не удалось. Приведем обзор результатов, полученных в этом направлении.

Формулируя проблему равенства для полугрупп (ассоциативных систем), А. Туэ в работе [101] особо выделил случай, когда полугруппа задается одним определяюшим соотношением вида $A=1$, где справа стоит пустое слово. Положительное решение проблемы Туэ для соотношений вида $A=1$ впервые было дано в [10]. При этом была доказана некоторая общая теорема, позволяюшая проблему равенства слов в полугруппе П, заданной системой определяюших соотношений специального вида

$$
A_{i}=1 \quad(i=1,2, \ldots, k)
$$


где длины $\left|A_{i}\right|$ слов $A_{i}$ все равны $l$, свести к проблеме равенства слов в группах, задаваемых тем же числом $k$ определяюших соотношений $B_{i}=1$, где $\left|B_{i}\right| \leqslant l$. Было доказано, что именно такое задание имеет максимальная подгруппа полугруппы П, порожденная всеми двусторонне обратимьпи элементами этой полугруппы. Был указан алгоритм, выдающий по заданию полугруппы П задание соответствующей максимальной подгрупшы $G_{\Pi}$, при условии что имеется алгоритм, решающий проблему равенства слов для груп, заданных $k$ нетривиальными определяюшими соотношениями вида $B_{i}=1$, где $\left|B_{i}\right| \leqslant l$. Отсюда в силу теоремы В. Магнуса о группах с одним определяюшим соотношением вытекает следуюший результат.

ТеОрема 4.1. Проблемь равенства (левой и правой) делимости слов разрешимы для любой полугруппь $\Pi_{1}$ с одним определяющим соотношением вида $A=1$.

Суть доказательства этой теоремы в [10] сводится к эффективному сведению рассматриваемых проблем в специальной полугруппе П с заданием (1) к проблеме равенства в максимальной подгруппе $G_{\text {П полугруппы }}$ П. При этом для систем соотношений вида (1) используются две операции расширения. Первая операция расштрения заключается в добавлении к определяюшим словам $A_{i}$ всех слов, которые получены из них циклическим сдвигом на так называемые двусторонние начала. Двусторонним началом относительно данной системы соотношений (1) называется слово, которое можно графически представить в виде произведения некоторых начал слов $A_{i}$ и в виде произведения некоторых конщов этих слов. Очевидно, любое двустороннее начало $E$ относительно системы (1) двусторонне обратимо в полугруппе П. Поэтому вместе с соотношением $A_{i}=E B_{i}=1$ в полугрупше П будет выполнено и соотношение $B_{i} E=1$, т.е. добавление указанных циклических сдвигов приводит к заданию той же групшы. Так как все добавляемые соотношения имеют ту же длину $l$, то эта операция расширения через конечное число шагов завершится.

В результате применения первой операции расширения к полугруппе $\Pi_{1}$, заданной одним исходным соотношением $A=1$, мы получим некоторое множество соотношений вида (1), где все левые части суть циклические сдвиги данного слова $A$ на некоторые его подслова $B_{j}$, которые двусторонне обратимы в $\Pi_{1}$. Пусть $B_{1}, B_{2}, \ldots, B_{r}$ - все полученные таким образом минимальные двусторонне обратимые отрезки слова $A$. Они порождают в $\Pi_{1}$ некоторую подгруппу $G_{1}$. Представив слово $A$ в виде $A=\varphi\left(B_{1}, B_{2}, \ldots, B_{r}\right)$, рассмотрим задание группы $G_{1}$ в новых образующих

$$
G_{1}=\left\langle\left\langle b_{1}, b_{2}, \ldots, b_{r} \mid \varphi\left(b_{1}, b_{2}, \ldots, b_{r}\right)=1\right\rangle\right\rangle .
$$

Использованная в [10] для общего случая вторая операция расширения по идее должна учитьвать всевозможные равенства между элементарными словами, полученными в результате применения первой операции расширения. В случае одного исходного определяюшего соотношения вида $A=1$ вторая операция расширения ничего нового не даст, так как в преобразованиях, производимых внутри данного элементарного слова $B_{j}$ участвуют не все порождающие рассматриваемой группы $G_{1}$, а только те $b_{i}$, для которых соответствуюшее слово $B_{i}$ входит в преобразуемое элементарное слово $B_{j}$. Поэтому порождающая $b_{j}$ в этих преобразованиях не участвует. Следовательно, по теореме о свободе 7.4 все возникающие при этом равенства будут равенствами в свободной группе, так что результат применения первой операции расширения уже будет замкнут и относительно второй операции расширения. Это означает, что $G_{1}$ уже 
будет максимальной подгруппой полугруппы П 1 . Это обстоятельство было недавно замечено в работе [110]. Поэтому для решения проблемы равенства в полугруппе П с соотношением $A=1$ после применения первой операции расширения в силу теоремы Магнуса о разрешимости проблемы равенства для групп с одним соотношением достаточно, следуя работе [10], воспользоваться следуюшей леммой.

Лемма 4.1. Пусть в $\Pi_{1}$ даны два слова $X=X_{1} E_{1} X_{2} E_{2} \ldots X_{r} E_{r} X_{r+1}$ и $Y=$ $Y_{1} D_{1} Y_{2} D_{2} \ldots Y_{s} D_{s} Y_{s+1}$, в которых выцелены все максимальные двусторонне обратимье подслова $X_{i}$ и $Y_{j}$.

Тогда $X=Y$ в полугруппе $\Pi_{1}$ в том и только том случае, если $r=s$ и при всех $і$ выполняются равенства $X_{i}=Y_{i}$ и $E_{i}=D_{i}$ в максимальной подгруппе $G$.

ОПРеДЕЛЕнИЕ. Слово $A$ мы называем гиперпростым, если никакое собственное начало слова $A$ не является его концом. Гиперпростое слово $A$ характерно тем, что в любом слове никакие два вхождения слова $A$ не пересекаются.

ОпреДЕЛЕниЕ. Соотношение $A=B$ при непустых $A$ и $B$ назовем симметричным. если слова $A$ и $B$ имеют обшее начало $T$, являюшееся также их обшим конщом, т.е. соотношение $A=B$ имеет один из двух видов:

$$
\begin{gathered}
T E T=T D T, \\
T E T=T,
\end{gathered}
$$

где слово $T$ гиперпросто.

Следуюшая теорема была доказана в [17].

Теорема 4.2. Для всякой полугруппь П, заданной одним симметричным определяющим соотношением $A=B$, можно указать определяющее соотношение $E=D$ такое, что $|E D|<|A B|$ и проблема равенства в П сводится к проблеме равенства для полугруппь $\Pi_{1}$, задаваемой одним соотношением $E=D$. При этом проблема левой (правой) делимости в П сводится к проблемам равенства и левой (соответственно, правой) делимости в $\Pi_{1}$.

ДокАЗАТЕльство. Искомое определяюшее соотношение $C=D$ по данному симметричному соотношению вида (2) или (3) с гиперпростьм $T$ строится с помошью следуюшей кодировки.

Перенумеруем все слова в алфавите полугруппы П, не содержашие вхождений гиперпростого слова $T$ :

$$
R_{1}, R_{2}, \ldots, R_{i}, R_{i+1}, \ldots .
$$

Слово $X$ назовем правильныц.м, если оно начинается и кончается словом $T$. Множество всех правильных слов полугруппы П взаимно однозначно отобразим на множество всех слов в алфавите

$$
b_{1}, b_{2}, \ldots, b_{i}, b_{i+1}, \ldots
$$

с помошью следуюшей функции $\varphi(X)$, определяемой индуктивно:

1) $\varphi(T) \rightleftharpoons \Lambda$;

2) если $X=X_{1} R_{i} T$, где $X_{1}$ есть правильное слово, то положим $\varphi(X) \rightleftharpoons \varphi\left(X_{1} b_{i}\right)$. 
Через П' обозначим полугруппу, заданную образующими (5) и определяющим соотношением

$$
\varphi(A)=\varphi(B) .
$$

Очевидно, для любых двух правильных слов $P$ и $Q$ переход $P \rightarrow Q$ есть элементарное преобразование полугрупшы П в том и только том случае, если $\varphi(P) \rightarrow \varphi(Q)$ есть элементарное преобразование в $\Pi^{\prime}$. Отсюда следует, что два правильных слова $U$ и $V$ равны в П в том и только том случае, если $\varphi(U)=\varphi(V)$ в $\Pi^{\prime}$. Далее нетрудно проверить все утверждения доказываемой теоремы.

Применяя многократно преобразование $\varphi$ к исходному симметричному соотношению $A=B$, мы придем к некоторому несимметричному соотношению. В частности, соотношение вида (3) приводится к соотношению вида $D=1$. Таким образом, из теорем 4.2 и 4.1 получается

СЛЕДСТВИЕ 4.2.1. Проблемы равенства (левой и правой делимости) разрешимы для любой полугруппы $\Pi_{1}$ с одним определяющим соотношением вида $T E T=T$.

В работе [9] исследовался вопрос о тождественных соотношениях в полугруппах, задаваемых любым числом определяюших соотношений вида $A_{i}=1$. Было доказано, что если полугруппа, имеющая такое задание, удовлетворяет некоторому нетривиальному тождеству, то она является группой, за исключением двух простых случаев, когда она изоморфна свободной моногенной полугруппе $\Pi_{0}=\langle a \mid \varnothing\rangle$ или полугруппе $\Pi_{1}=\langle a, b \mid a b=1\rangle$. Полугруппа $\Pi_{0}$ коммутативна, а полугруппа $\Pi_{1}$ удовлетворяет тождественному соотношению

$$
x y^{2} x y x^{2} y^{2} x=x y^{2} x^{2} y x y^{2} x
$$

причем это тождество является кратчайшим в полугруппе $\Pi_{1}$ по длине левых и правых частей [10].

ЛЕМма 4.2. Проблема равенства слов разрешима в любой полугруппе П, заданной соотношением $A=B$ при $|A|>|B|$, если слово $A$ гиперпросто.

ДокАЗАТЕльСтво. В такой полугруппе П каждое слово $X$ можно в результате конечного числа подстановок слова $B$ вместо вхождений $A$ преобразовать в равное ему

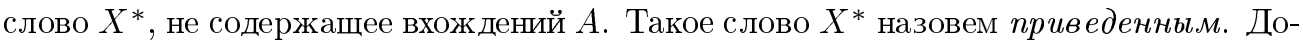
статочно проверять равенство в П для приведенных слов. Легко убедиться, что два приведенных слова равны в П только в том случае, если они совпадают графически. Допустим, что два разных приведенных слова $C$ и $D$ равны в П. Рассмотрим кратчайшую последовательность элементарных преобразований, переводящую $C$ в $D$. В ней первое преобразование будет заменой $B$ на $A$, причем возникшее последним вхождение слова $A$ в преобразуемое слово не будет затронуто в силу своей гиперпростоты до тех пор, пока оно не заменится обратно на $B$; причем такая замена $A$ на $B$ обязательно произойдет, так как по условию $A$ не входит в $D$. Но это противоречит условию минимальности рассматриваемой последовательности.

Очевидно, указанньй алгоритм имеет линейную сложность.

На пути решения проблемы равенства слов для полугрупп с одним определяюшим соотношением вида $A=B$ с непустыми $A$ и $B$ в работе [7] было найдено достаточное 
условие вложимости в группу для полугруп, задаваемых при помоши определяющих соотношений. Эти полугруппы выделяются с помощью так называемых систем соотношений без левьх и правых циклов.

ОПРЕДЕЛЕНИЕ. Пусть дана некоторая система образующих и определяющих соотношений

$$
\left\langle a_{1}, \ldots, a_{m} \mid\left\{A_{i}=B_{i} \mid i=1, \ldots, m\right\}\right\rangle,
$$

где $A_{i}, B_{i}$ - непустые слова. Левым графом этой системы соотношений называется граф, вершинами которого являются все буквы алфавита, а ребрами - все неупорядоченные пары букв $\left\{a_{j_{i}}, a_{r_{i}}\right\}$, где $a_{j_{i}}$ и $a_{r_{i}}$ суть начальные буквы левой и правой частей определяюшего соотношения $A_{i}=B_{i}$. Если вместо начальных букв слов $A_{i}$ и $B_{i}$ брать их концевые буквы, то получим определение правого графа данной системы определяюших соотношений.

Мы говорим, что данная система определяющих соотношений не имеет левых $(n p a в b x)$ uиклов, если нет циклов в соответствуюшем левом (правом) графе.

Для случая одного определяюшего соотношения $A=B$ это условие сводится к требованию, чтобы слова $A$ и $B$ были непустыми и чтобы они начинались (соответственно, оканчивались) разными буквами, т.е. чтобы определяюшее соотношение было несократимо слева (соответственно, справа).

В работе [10] доказана следующая теорема о вложении.

ТЕОрема 4.3. Если система определяющих соотношений (6) данной полугруппь П не имеет левых и правых ииклов, то в полугруппе П выполнены оба правила сокращения и она естественным образом вложена в группу, заданную теми же определяющими соотношениями.

Отсюда в силу указанного выше результата В. Магнуса получается

СлЕДСТВИЕ 4.3.1. Существует алгоритм, решающий проблему равенства слов для полугрупп, заданных одним несократимым слева и справа соотношением вида $A=B$ при непустых $A$ и $B$.

Другим следствием теоремы 4.3 является вложимость в группу любой полугруппы, заданной одним определяюшим соотношением с использованием двустороннего правила сокрашения. Легко проверить, что полугруппа с двумя определяюшими соотношениями

$$
\Pi_{2} \rightleftharpoons\langle a, b, c, d, e \mid a b=c d, a e b=c e d\rangle
$$

не вкладьвается в группу, хотя в ней выполнено правило двустороннего сокрашения. Действительно, в групе с такими же соотношениями выполнено равенство $a e^{2} b=$ $c e^{2} d$, в то время как в полугруппе $\Pi_{2}$ оно не вьполнено.

Необходимым условием вложимости данной полугрупшы в группу является требование, чтобы в ней выполнялись следуюшие правила сокрашения:

1. $A X=A Y \Longrightarrow X=Y$,

2. $X A=Y A \Longrightarrow X=Y$. 
Полугруппа, в которой оба эти правила выполнены, называется полугруппой с двусторонним сокращением.

В работе [4] исследовалась роль правила сокрашения при задании полугрупп с помошью определяюших соотношений. Для полугрупп с правилом сокращения возможны два способа задания с помощью определяющих соотношений. Можно требовать, чтобы полугрупп задавалась системой определяющих соотношений, из которых уже вытекает справедливость соответствующего правила сокращения. А можно добавить это правило сокрашения в качестве дополнительного правила вывода истинных равенств. Если не ограничивать числа определяющих соотношений, то разница здесь не является сушественной. Но для конечно определенных полугрупп это оказывается существенным. В работе [10] было установлено, что при задании конечно определенных полугрупп с использованием правила правостороннего (левостороннего) сокращения это правило, вообше говоря, нельзя заменить конечным числом дополнительных определяюших соотношений. Доказано, что в полугрупе П, заданной образуюшими элементами

$$
a, b, t_{1}, t_{2}, p_{1}, p_{2}
$$

и определяющими соотношениями

$$
a t_{1}=t_{1} b, \quad b t_{2}=t_{2} b, \quad p_{1} t_{1}=t_{2} p_{1} t_{2}, \quad p_{2} t_{1}=t_{1} p_{2}, \quad t_{2} p_{2}=p_{2} t_{2} t_{1},
$$

для любого целого положительного числа $k$ вьполняется равенство

$$
p_{1} a^{k} p_{2}=t_{2} p_{1} b^{k} p_{2} t_{2}
$$

причем каждое из этих равенств в соответствующих обозначениях должно входить в систему определяющих соотношений любого задания полугруппы П с помошью определяющих соотношений без использования правила правостороннего сокращения.

Для конечно определенных полугрупп, удовлетворяюших правилу левостороннего (правостороннего) сокращения, при условии, что все определяюшие слова непусты, проблема равенства слов тесно связана с так называемой проблемой распознавания левой (правой) делимости.

ОПРЕДЕЛЕНИЕ. Говорят, что в полугруппе П слово $A$ делится на слово $B$ слева (справа), если существует такое слово $X$, что в П выполнено равенство $A=B X$ (соответственно, $A=X B$ ).

ПРОБЛЕМА ЛЕВОЙ (ПРАВОЙ) ДЕЛИМОСТИ для данной полугруппъ заключается в отыскании алгоритма, с помощью которого можмно бъло бъ для любъх двух слов $А$ и В узнавать, делится ли слово А на слово В слева (справа), и в случае положительного ответа указать искомое частное $X$.

Очевидно, для разрешимости проблемы левой (правой) делимости для данной полугруппы достаточно иметь алгоритм для распознавания делимости на одну букву с нужной стороны.

ЛЕМма 4.4. Пусть в полугруппе П с системой определяющих соотношений (6), где все $A_{i}, B_{i}$ - непустые слова, выполнено правило левостороннего (правостороннего) сокращения. Тогда из разрешимости в ней проблемы распознавания левой (соответственно, правой) делимости следует разрешимость в ней и проблемы равенства. 
ДоКАЗАТЕЛЬСТВО. В силу симметрии ограничимся случаем, когда в П вьполнено правило левостороннего сокращения. Сначала заметим, что никакое непустое слово не равно 1 в П. Для проверки равенства двух данных слов $A$ и $B$ мы сначала проверим делимость $A$ на $B$. Если не делится, то данные слова не равны. В случае делимости найдем такое слово $X$, что в П вьполнено равенство $A=B X$. Если при этом $A=B$, то из $B=B X$ по правилу левостороннего сокращения получим $1=X$ в П, т.е. слово $X$ должно быть пусто. Лемма 4.4 доказана.

В работе [14] исследовалась проблема распознавания делимости произвольного слова $X$ слева (справа) на данную букву в полугруппах, заданных определяющими соотношениями без левых (правых) циклов. Был предложен простой алгоритм $\mathfrak{A}$, который для любой полугруппы П, заданной системой определяюших соотношений без левых (правых) циклов, выдает кратчайшую последовательность элементарных преобразований полугруппы П, переводящую данное слово $X$ в некоторое слово, начинающееся (кончаюшееся) данной буквой $b$, при условии, что $X$ делится в полугрупте П на $b$ слева (справа). Там же было доказано, что если полугруппа П задана системой определяющих соотношений без левых (правых) циклов, то для любых двух равных в П слов $X$ и $Y$ сушествует единственная, с точностью до перестановки независимых преобразований, последовательность элементарных преобразований без поворотов, переводяшая слово $X$ в слово $Y$.

Напомним определение левого разложсения $R_{l}(a X, b)$ слова $a X$ относительно буквы $b$ и левого разложения $R_{l}(b X, a)$ слова $b X$ относительно буквы $a$ [14; c. 615$]$. Для простоты рассмотрим случай, когда рассматриваемая полугруппа задана одним соотношением. Такое разложение существует всегда, если полугруппа П имеет только две порождающие $a$ и $b$. В случае большего числа порождаюших разложение $R_{l}(c X, d)$ имеет смысл рассматривать, только когда одна из двух букв $c$ и $d$ совпадает с $a$, а другая $-\mathrm{c} b$.

Разложения $R_{l}(a X, b)$ и $R_{l}(b X, a)$, которые мы определяем последовательно шаг за шагом, имеют вид

$$
* H_{1} * H_{2} * \cdots * H_{k} * E * Z
$$

где $H_{1} H_{2} \ldots H_{k} E Z$ графически совпадает со словом $a X$ (соответственно, со словом $b X)$, слово $E$ называется головкой, а слова $H_{i}$ называются компонентами разложения (7). Слово $Z$ может быть пусто. Компоненты $H_{i}$ являются непустыми собственными началами левой части $a A$ или правой части $b B$ определяюшего соотношения полугруппы П. Компоненту $H_{i}$ разложения $(7)$ мы назьваем $A$-компонентой ( $B$-компонентой), если она есть начало $a A$ (соответственно, $b B$ ). Головка $E$ совпадает c $a A$ или с $b B$. Разложение (7) может не иметь головки.

Определения разложений $R_{l}(a X, b)$ и $R_{l}(b X, a)$ аналогичны. Поэтому ограничимся рассмотрением $R_{l}(a X, b)$.

Пусть $E$ есть максимальное общее начало слов $a X$ и $a A$, т.е. $a X=E Z$ и $a A=E D$ при некоторых $Z$ и $D$. Если слово $D$ пусто, то мы полагаем по определению

$$
R_{l}(a X, b)=* E * Z
$$

и назьваем подслово $E$ головкой этого разложения. 
Пусть $D$ непусто. В таком случае мы обозначим слово $E$ через $H_{1}$ и назовем его первым крылом искомого разложения. Если при этом $Z$ пусто, то положим

$$
R_{l}(a X, b)=* H_{1} *
$$

Пусть $Z$ непусто. Тогда $Z$ и $D$ начинаются с разных букв. Если хотя бы одна из этих букв отлична от $a$ и $b$, то разложение $R_{l}(a X, b)$ не сушествует по определению. Пусть либо $Z \mp a Y$ при некотором $Y$ и слово $D$ начинается с $b$, либо $Z \mp b Y$ при некотором $Y$ и слово $D$ начинается с $a$. Пусть для определенности, $Z=b Y$ при некотором $Y$ и слово $D$ начинается с $a$. В таком случае аналогично предыдущему находим максимальное общее начало $E_{1}$ слов $b Y$ и $b B$, т.е. $b Y=E_{1} Z_{1}$ и $b B=E_{1} D_{1}$ при некоторых $Z_{1}$ и $D_{1}$.

Если слово $D_{1}$ пусто, то мы полагаем по определению

$$
R_{l}(a X, b)=* H_{1} * E_{1} * Z_{1}
$$

и называем слово $E_{1} \mp b B$ головкой этого разложения.

Пусть $D_{1}$ непусто. Тогда обозначим $E_{1}$ через $H_{2}$ и назовем его вторым крылом искомого разложения. Если при этом $Z_{1}$ пусто, то положим

$$
R_{l}(a X, b)=* H_{1} * H_{2} * .
$$

Если $Z_{1}$ непусто, то $Z_{1}$ и $D_{1}$ начинаются с разных букв. Если хотя бы одна из этих букв отлична от $a$ и $b$, то опять разложение $R_{l}(a X, b)$ не сушествует по определению. В противном случае мы продолжим процесс нахождения левого разложения слова $a X$, рассматривая максимальное общее начало $E_{2}$ слова $Z_{1}$ и того определяюшего слова, которое начинается с первой буквы слова $D_{1}$.

Так как $|a X|>|Z|>\left|Z_{1}\right|$, то через конечное число шагов мы либо придем к случаю, когда разложение $R_{l}(a X, b)$ не сушествует по определению, либо получим головку $E_{k}$ разложения и при этом искомое разложение будет иметь вид

$$
R_{l}(a X, b)=* H_{1} * H_{2} * \cdots * H_{k} * E_{k} * Z_{k},
$$

либо придем к случаю пустого слова $Z_{k}$ и разложению без головки

$$
R_{l}(a X, b)=* H_{1} * H_{2} * \cdots * H_{k} * .
$$

Этим завершается индуктивное определение разложений $R_{l}(a X, b)$ и $R_{l}(b X, a)$ для случая одного соотношения. В обшем случае системы соотношений без левых циклов при построении разложения $R_{l}(a X, b)$ на каждом шаге надо сначала проверить, связаны ли путем в левом графе данные две буквы $a$ и $b$. Если такого пути нет, то разложение $R_{l}(a X, b)$ не существует по определению. Если же буквы $a$ и $b$ связаны некоторьм путем, то надо рассмотреть разложение $R_{l}(a X, c)$ для той буквы $c$, которая связана с $а$ некоторым ребром на этом пути. Это означает, что среди определяющих соотношений есть соотношение вида $a A=c C$, причем оно будет единственным в силу отсутствия циклов в левом графе рассматриваемой системы соотношений.

Легко доказать, что правило левостороннего сокращения выполняется для систем соотношений (6) без левых циклов, в том числе для случая одного несократимого слева соотношения. 
ЛЕмма 4.3. Пусть полугруппа П задана одним несократимылм слева соотношением $a E=b D$ в некотором алфавите. Если последовательность әлементарньх преобразований полугруппь П

$$
e X=X_{0} \rightarrow X_{1} \rightarrow \cdots \rightarrow X_{k}=e Y
$$

является кратчайшей для данных слов еX и еY, то в этих преобразованиях первая буква е преобразуемого слова не затрагивается, т.е. в ней фактически слово $X$ преобразуется в $Y$.

ДокАЗАТЕЛьство. Доказательство проведем индукцией по числу $k$ элементарных преобразований в данной кратчайшей последовательности. Можно считать, что буква $е$ совпадает с одной из букв $a$ или $b$. Пусть для определенности данная кратчайшая последовательность имеет вид

$$
a X=X_{0} \rightarrow X_{1} \rightarrow \cdots \rightarrow X_{k}=a Y
$$

Допустим, что в (8) первая буква затрагивается. Выделив в (8) первое и последнее вхождения слов, начинаюшихся с отличной от $a$ буквы $b$, получим, что последовательность (8) состоит из трех частей

$$
\begin{gathered}
a X \mp X_{0} \rightarrow X_{1} \rightarrow \cdots \rightarrow X_{r-1} \mp a E U \rightarrow b D U \\
b D U \rightarrow X_{r+1} \rightarrow \cdots \rightarrow X_{t} \mp b D V
\end{gathered}
$$

и

$$
b D V \rightarrow a E V=X_{t+1} \rightarrow \cdots \rightarrow X_{k}=a Y
$$

причем в (9) и (11) буква $а$ затрагивается только в одном выделенном преобразовании. По индуктивному предположению в последовательности (10) буква $b$ и все входящие в подслово $D$ буквы не затрагиваются. Это означает, что в (10) по сушеству мы имеем последовательность элементарных преобразований

$$
U \rightarrow \cdots \rightarrow V
$$

Используя последовательности (9), (12) и (11), мы можем построить более короткую, чем (8), последовательность элементарных преобразований, переводяшую слово $a X$ в $a Y$, исключив последнее преобразование в (9) и первое преобразование в (11). Но это противоречит предположению, что последовательность (8) была кратчайшей.

В обшем случае системы соотношений (6) без левых циклов доказательство проводится аналогично. При этом перед разделением кратчайшей последовательности (8) на три части (9), (10) и (11) нужно убедиться, что в первом и последнем из преобразований последовательности (8), затрагивающих начальную букву, используется одно и то же соотношение из (6), что следует из отсутствия циклов в левом графе системы соотношений (6). 
Алгоритм $\mathfrak{A}[14 ;$ с. 620]. Пусть полугруппа П задана системой определяющих соотношений без левых циклов и дана пара $(a X, b)$, где буквы $a$ и $b$ различны. Из определения левого разложения $R_{l}(a X, b)$ непосредственно вытекает простой алгоритм для распознавания по данной паре $(a X, b)$, сушествует или нет разложение $R_{l}(a X, b)$, и построения этого разложения в случае, когда оно сушествует.

Алгоритм $\mathfrak{A}$ в применении к данной паре $(a X, b)$ предписывает сначала найти левое разложение $R_{l}(a X, b)$. Однозначность разложения $R_{l}(a X, b)$ следует непосредственно из его определения.

I. Если слово $a X$ делится на $b$ в полугруппе П, то из анализа кратчайшей последовательности, реализующей эту делимость, в силу леммы 4.3 и определения левого разложения следует существование разложения $R_{l}(a X, b)$ с некоторой головкой. Поэтому если такое разложение не существует или оно не имеет головки, то слово $a X$ не делится на $b$ слева в П.

II. Если же $R_{l}(a X, b)$ имеет головку, скажем, оно имеет вид

$$
R_{l}(a X, b)=* H_{1} * H_{2} * \cdots * H_{k} * E * Z,
$$

где $E=D$ есть определяюшее соотношение П, то алгоритм предписьвает заменить в слове $a X$ головку $E$ словом $D$, преобразуя тем самым слово $a X=H_{1} H_{2} \ldots H_{k} E Z$ в слово $Y=H_{1} H_{2} \ldots H_{k} D Z$.

III. Если при этом $Y$ не начинается с буквы $b$, скажем $Y=c Y_{1}$, то предписывается повторить описанную процедуру с парой $\left(c Y_{1}, b\right)$ и т. д.

Следуюшая гипотеза была выдвинута первым автором данной статьи еше в 1976 году.

ГИПоТЕЗА. Проблема левой (правой) делимости, а следовательно, и проблема равенства слов, разрешима в любой полугруппе без левых (правых) ииклов, причем, мы полагаем, что положительное решение этой проблемы может бъть получено в результате применения описанного алгоритма $\mathfrak{A}$. Требуется найти подходящие критерии “зачикливания" работы алгоритма $\mathfrak{A}$, т.е. описания тех случаев, когда алгоритм будет работать бесконечно.

Следует отметить, что многие из полученных в последниегоды результатов по проблемам равенства и делимости для полугрупп с одним определяющим соотношением основывались на алгоритме $\mathfrak{A}$. Приведем некоторые из таких результатов.

ТЕОРема 4.4. Если полугруппа П задана п определяющими соотношениями без левых (правых) ииклов

$$
A_{i}=B_{i} \quad(i=1,2, \ldots, n),
$$

то можсно указать такую систему соотношений

$$
\overline{A_{i}}=\overline{B_{i}} \quad(i=1,2, \ldots, n)
$$

в алфавите из $n+1$ буквы, что каждая из проблем равенства и левой (соответственно, правой) делимости для П сводится $\kappa$ той же проблеме для полугруппь $\bar{\Pi}$, заданной соотношениями (14), причем $\left|A_{i}\right|=\left|\overline{A_{i}}\right|,\left|B_{i}\right|=\left|\overline{B_{i}}\right|$. 
ДокАЗАТЕЛьство. Пусть система (13) не имеет левых циклов, а $M$ есть алфавит полугруппы П. Рассмотрим левый граф Г системы (13). Буквы, являющиеся началами слов $A_{i}$ или $B_{i}$, назовем левьци. Если буква из $M$ не левая, то она является изолированной вершиной в $\Gamma$. В каждой связной компоненте $\Gamma_{j}(j=1,2, \ldots, k)$ графа $\Gamma$ отметим по одной букве $c_{j}$. Для любого слова $X$ в алфавите $M$ через $\bar{X}$ обозначим результат замены в $X$ всех вхождений отмеченных букв $c_{j}$ буквой $c_{1}$ из первой компоненты. Так как $Г$ не содержит циклов, то полученная таким образом система соотношений (14) будет иметь левый граф $\bar{\Gamma}$ без циклов. Граф $\bar{\Gamma}$ состоит из одной компоненты и потому имеет $n+1$ вершину.

Докажем, что проблема левой делимости в П сводится к той же проблеме для $\bar{\Pi}$. Как было отмечено выше, достаточно указать алгоритм, распознаюший делимость слева в П данного слова $X$ на любую букву $b \in M$.

Если первая буква данного слова $X$ и буква $b$ лежат в различных компонентах графа $\Gamma$, то $X$ не делится слева на $b$. Пусть эти буквы лежат в одной компоненте и не совпадают. Согласно пункту I описания алгоритма $\mathfrak{A}$ можем считать, что левое разложение слова $X$ в полугрупе П относительно буквы $b$ имеет вид

$$
R_{l}(X, b)=H_{1} * H_{2} * \cdots * H_{t} * E * U,
$$

где $E$ - головка этого разложения. В этом случае первая буква слова $\bar{X}$ будет отлична от $\bar{b}$, так как буквы из одной компоненты не склеивались. Тогда левое разложение слова $\bar{X}$ в полугруппе $\bar{\Pi}$ будет иметь вид

$$
R_{l}(\bar{X}, \bar{b})=\overline{H_{1}} * \overline{H_{2}} * \cdots * \overline{H_{t}} * \bar{E} * \bar{U} .
$$

При этом один шаг применения алгоритма $\mathfrak{A}$ к паре $(X, b)$ в П дает

$$
X \rightarrow H_{1} H_{2} \ldots H_{t} S U \rightleftharpoons X_{1},
$$

где $E=S$ - соотношение системы (13), а один шаг применения $\mathfrak{A}$ к паре $(\bar{X}, \bar{b})$ в $\bar{\Pi}$ дает

$$
\bar{X} \rightarrow \overline{H_{1}} \overline{H_{2}} \ldots \overline{H_{t}} \bar{S} \bar{U} .
$$

Таким образом, если алгоритм $\mathfrak{A}$ по паре $(X, b)$ выдает в П последовательность элементарных преобразований

$$
X \rightarrow X_{1} \rightarrow X_{2} \rightarrow \cdots \rightarrow X_{\lambda}=b Z,
$$

то в $\bar{\Pi}$ алгоритм $\mathfrak{A}$ выдает последовательность

$$
\bar{X} \rightarrow \overline{X_{1}} \rightarrow \overline{X_{2}} \rightarrow \cdots \rightarrow \overline{X_{\lambda}}=\overline{b Z} .
$$

Поэтому если проблема распознавания делимости слева в $\bar{\Pi}$ разрешима, то мы имеем следуюший алгоритм для распознавания делимости слева в П слова $X$ на букву $b$. Если $\bar{X}$ не делится слева на $\bar{b}$ в $\bar{\Pi}$, то тем более $X$ не делится слева на $b$ в П. Если же $\bar{X}$ делится слева на $\bar{b}$ в $\bar{\Pi}$, то, применив алгоритм $\mathfrak{A}$ к паре $(\bar{X}, \bar{b})$, через некоторое число шагов $\lambda$ получим слово, начинаюшееся с буквы $\bar{b}$. Тогда в силу сказанного выше 
алгоритм $\mathfrak{A}$ для П, начав работу с пары $(X, b)$, либо выдаст через $\lambda$ шагов слово, начинающееся с $b$, либо остановится на слове, не начинающемся с $b$. В последнем случае слово $X$ не делится слева на $b$ в П.

Допустим, что в $\bar{\Pi}$ разрешима проблема равенства. Пусть надо проверить, равны ли в П два данных слова $X$ и $Y$. Проведем индукцию по длине $Y$. Так как все определяющие слова полугруппы П непусты, то можно считать, что $|Y|>0$. Сначала проверим равенство $\bar{X}=\bar{Y}$ в $\bar{\Pi}$. Если $\bar{X} \neq \bar{Y}$ в $\bar{\Pi}$, то $X \neq Y$ в П. Пусть $\bar{X}=\bar{Y}$ в $\bar{\Pi}$. В силу правила сокращения можно считать, что $X$ и $Y$ начинаются с разных левых букв $a$ и $b$, принадлежащих одной компоненте $\Gamma_{i}$. Тогда начальные буквы $\bar{a}$ и $\bar{b}$ слов $\bar{X}$ и $\bar{Y}$ также различны. Применение алгоритма $\mathfrak{A}$ к паре $(\bar{X}, \bar{b})$ в $\bar{\Pi}$ приведет к ответу $\overline{b Z}$ через некоторое число шагов $\lambda$. Тогда по доказанному вьше, применяя алгоритм $\mathfrak{A} \mathrm{k}$ паре $(X, b)$ в $\Pi$, не позднее чем через $\lambda$ шагов мы либо убедимся, что $X$ не делится на $b$ слева в П, либо получим слово $b Z$. В последнем случае мы будем иметь в П равенство $X=b Z$. Тогда, сократив на $b$ слева, получим

$$
X=Y \text { в } \Pi \Longleftrightarrow Z=Y_{1} \text { в } \Pi,
$$

где $Y=b Y_{1}$. Так как $\left|Y_{1}\right|<|Y|$, то по индуктивному предположению мы можем проверить равенство $Z=Y_{1}$ в П. Теорема доказана.

В совместной работе [18] проблемы равенства и левой (правой) делимости в полугруппах с одним определяющим соотношением были сведены к аналогичной проблеме для случая, когда это определяюшее соотношение несократимо хотя бы с одной стороны.

Пусть полугруппа П задана одним соотношением $A=B$ в алфавите $M$ из $m$ букв. Пусть $E_{1}, E_{2}, \ldots, E_{n}$ суть все слова длины $k$ в алфавите $M$ и $n=m^{k}$.

Рассмотрим новый алфавит $e_{1}, e_{2}, \ldots, e_{n}$. Положим $\tau_{k}\left(E_{i}\right) \rightleftharpoons e_{i}$, где $e_{i}-$ буква нового алфавита; $\tau_{k}(X) \rightleftharpoons e_{i} \tau_{k}(Y)$, если $X=a Y$, где $a \in M$ и $E_{i}$ - начало $X$ длины $k$. Кроме того, для любого слова $X$ длины $<k$ положим $\tau_{k}(X) \rightleftharpoons \Lambda$, где $\Lambda$ - пустое слово.

Отметим следуюшее очевидное свойство кодировки $\tau_{k}$. Пусть $P, Q$ - произвольные слова в алфавите $M$. Если $|Q| \geqslant k-1$, то $\tau_{k}(P Q) \mp \tau_{k}\left(P Q_{1}\right) \tau_{k}(Q)$, где $Q_{1}$ - начало слова $Q$ длины $k-1$. Аналогично, если $|P| \geqslant k-1$, то $\tau_{k}(P Q) \mp \tau_{k}(P) \tau_{k}\left(P_{1} Q\right)$, где $P_{1}-$ конец длины $k-1$ слова $P$.

ЛЕмма 4.5. Пусть полугруппа П задана сократимым с обеих сторон соотношением $A=B, C$ есть максимальное общее начало, а $D$ - максимальньй общий конец слов $А$ и В. Тогда каждая из проблем равенства, левой и правой делимости для П сводится к той же проблеме для полугруппь

$$
\Pi_{1} \rightleftharpoons\left\langle e_{1}, e_{2}, \ldots, e_{n} \mid \tau_{k}(A)=\tau_{k}(B)\right\rangle
$$

$2 \partial e$

$$
k \rightleftharpoons 1+\min (|C|,|D|) .
$$

Если $|C|=|D|$, то проблемы равенства, левой и правой делимости для П разрешимы. 
ДокАЗАТЕЛЬСтво. Очевидно, соотношение $\tau_{k}(A)=\tau_{k}(B)$ несократимо слева, если $|C| \leqslant|D|$, и оно несократимо справа, если $|C| \geqslant|D|$. Не ограничивая обшности, можем считать, что $|B| \leqslant|A|$ и $|C| \leqslant|D|$. Если $B$ является двусторонним началом $A$, то можно применить следствие 4.2.1.

Пусть $B$ не является двусторонним началом $A$. Тогда $|C|<|D|, k=1+|C| \leqslant|D|$, поэтому слова $\tau_{k}(A)$ и $\tau_{k}(B)$ непусты. Из $k>|C|$ следует, что соотношение $\tau_{k}(A)=$ $\tau_{k}(B)$ несократимо слева.

Для любых слов $P, Q$ полугруппы П имеем:

$$
\begin{aligned}
\tau_{k}(P A Q) & =\tau_{k}(P C) \tau_{k}(A) \tau_{k}\left(D_{1} Q\right), \\
\tau_{k}(P B Q) & =\tau_{k}(P C) \tau_{k}(B) \tau_{k}\left(D_{1} Q\right),
\end{aligned}
$$

где $D_{1}$ - конец слова $D$ и $\left|D_{1}\right|=|C|=k-1$. Отсюда легко следует, что для любых слов $X$ и $Y$ длины $\geqslant k$ выполнены эквивалентности

$$
X=Y \text { в } \Pi \Longleftrightarrow \tau_{k}(X)=\tau_{k}(Y) \text { в } \Pi_{1},
$$

( $X$ делится на $Y$ слева (справа) в П)

$$
\Longleftrightarrow\left(\tau_{k}(X) \text { делится на } \tau_{k}(Y) \text { слева (справа) в } \Pi_{1}\right) \text {. }
$$

Если же длина одного из слов $X$ и $Y$ меньше $k$, то имеем

$$
X=Y \text { в } \Pi \Longleftrightarrow X=Y,
$$

( $X$ делится на $Y$ слева (справа) в $\Pi) \Longleftrightarrow(Y$ есть начало (конец) $X)$.

Если $|C|=|D|$, то, очевидно, соотношение $\tau_{k}(A)=\tau_{k}(B)$ будет несократимо и справа. Лемма доказана.

Теорема 4.5. Проблема равенства (левой или правой делимости) в произвольной полугруппе с одним определяющим соотношением сводится к той жсе проблеме для некоторой полугруппь, имеющей задание одного из следующих двух видов

$$
\langle a, b \mid a A a=b B a\rangle
$$

uภu

$$
\langle a, b \mid a=b B a\rangle,
$$

где $a, b$ - порождающие, $а$ А и $B$ - произвольные слова.

ДокАЗАТЕЛЬСТво. Пусть полугруппа П задана одним определяющим соотношением. В силу леммы 4.5 проблемы равенства (левой и правой делимости) в П сводятся к тем же проблемам в некоторой полугруппе $\Pi_{1}$ с одним определяюшим соотношением. Случай несократимого справа соотношения легко сводится к случаю соотношения, несократимого слева, так как мы можем переписать данное соотношение справа налево и в полученной полугруппе исследовать равенство или делимость слева для слов, симметричных данным. Кроме того, в силу теоремы 4.4 можно считать, что П 1 имеет 
только две образующие. Тогда, с точностью до переименования букв, полугруппа П 1 имеет задание одного из двух видов (15) или (16).

Заметим, что теорема 4.5 впервые была доказана в 1978 году в работе [17]. Позже аналогичное утверждение было опубликовано без доказательства в работе [41].

В [77] было получено решение проблем равенства и делимости для полугрупп, заданных одним соотношением вида $a=A$, где $A$ - слово в двубуквенном алфавите. Это решение основьвалось на результате О. А. Саркисян [92] о разрешимости проблемы левой (правой) делимости для любой полугрупшы, заданной системой определяющих соотношений без левых и правых циклов. Однако, как недавно было обнаружено, приведенное в [92] доказательство этого впечатляющего утверждения содержит сушественньй пробел и О. А. Саркисян в настоящее время не знает как восполнить этот пробел. Хотя у нас нет серьезных сомнений в истинности указанных утверждений Г.У. Оганесяна и О.А. Саркисян, их пока следует считать недоказанньми.

По той же причине теорему 3 из работы [18] следует считать доказанной в следующей ослабленной редакции.

ТЕОРЕма 4.6. Пусть полугруппа П задана определяющим соотношением $A=B$, где слово $B$ гиперпросто и оно входит в слово $A$, но не является его кониом. Тогда для П разрешима проблема равенства.

Здесь дополнительное условие, что $B$ не является конщом $A$, позволяет сохранить приведенное в [18] доказательство, убрав только ссылку на не доказанный еще результат об уравнениях вида $b A a=a$. В доказательстве этой теоремы сушественно используется описанный в работе [14] алгоритм $\mathfrak{A}$ и следующая лемма, которая интересна сама по себе.

Лемма 4.6. Пусть полугруппа П задана определяющим соотношением а $A_{1}=$ $b B_{1}$, где а и $b$-буквы и слово $B=b B_{1}$ гиперпросто. Если слово bF есть собственный конец слова $B$, то при любом $V$ слово $b F V$ не делится в П на а слева.

ДокАЗАТЕльство. Рассмотрим кратчайшую последовательность элементарных преобразований полугруппы П вида

$$
a Z \rightarrow \cdots \rightarrow b F V
$$

где $b F$ - собственный конец слова $B$. В этой последовательности выделим первое появление буквы $b$ в начале преобразуемого слова:

$$
a Z \rightarrow \cdots \rightarrow a A_{1} Z_{1} \rightarrow b B_{1} Z_{1} \rightarrow \cdots \rightarrow b F V .
$$

В силу гиперпростоты $B$ максимальное общее начало слов $B$ и $b F$ короче $b F$, т.е. $F=F_{1} F_{2}, B_{1}=F_{1} B_{2}$, где $F_{2}$ - непустой конец $b B_{1}$, причем слова $B_{2}$ и $F_{2}$ начинаются с разных букв, т.е. одно из них начинается с $b$, а другое с $a$. Сократив конец последовательности (17) слева на слово $b F_{1}$, получим

$$
B_{2} Z_{2} \rightarrow \cdots \rightarrow F_{2} V .
$$

Но это противоречит условию минимальности последовательности (17).

Приведем еше два результата о разрешимости проблемы равенства для полугрупп, заданных одним определяюшим соотношением $A=B$, где слово $B$ гиперпросто и не входит в $A$. Они также доказаны с использованием алгоритма $\mathfrak{A}$.

Следуюшая теорема доказана в работе [15]. 
Теорема 4.7. Проблемы равенства и левой делимости разрешимы для полугрупп, заданных одним определяющим соотношением вида $a A=b B$, где $b B$ гиперпросто, не входит в аА и удовлетворяет одному из условий

1) никакое собственное начало слова $b B$ не является концом $а A$,

2) никакой собственный конеи слова $b B$ не является началом аА.

Теорема 4.8 [109]. Пусть полугруппа П задана одним определяющим соотношением $A=B$, где слово $B$ гиперпросто и не входит в $A$. Если при этом выполнено неравенство $|A| \geqslant|B|^{2}$, то в П разрешима проблема равенства.

Следуюшая теорема является усилением теоремы 4.7 и может быть доказана аналогично с использованием леммы 4.6 .

Теорема 4.9. Используя алгоритм $\mathfrak{A}$, можно решить проблему левой делимости (а значит, и проблему равенства) для полугруппь

$$
\Pi=\langle a, b, \ldots \mid a A=b B\rangle,
$$

где $b B$ гиперпросто и не входит ни в какое произведение вида $A_{1} A_{2} \ldots A_{k}$, әде все $A_{i}$ суть концы слова аА.

\section{5. Группы с условием малого сокращения}

В работе [31] М. Дэн предложил простой алгоритм решения проблемы равенства слов для фундаментальных групп замкнутых ориентируемых двумерных многообразий. Метод М. Дэна позже был распространен на широкие классы групп, удовлетворяюшие условию малого сокращения определяющих слов. Пусть конечно определенная группа $G$ задана образуюшими и определяюшими соотношениями:

$$
\left\langle\left\langle a_{1}, \ldots, a_{n} \mid R_{i}=1, \ldots, R_{m}=1\right\rangle\right\rangle .
$$

Очевидно, мы можем считать, что все определяюшие слова $R_{i}$ циклически несократимы. Через $\mathfrak{R}_{G}$ обозначим множество всех определяюших слов в (1). Представление (1) назьвается симметризованныц.м. если множество $\mathfrak{R}_{G}$ замкнуто относительно циклических сдвигов и перехода к обратному слову.

Пусть задание (1) симметризованное. Слово $K$ называется куском относительно множества $\mathfrak{R}_{G}$, если в $\mathfrak{R}_{G}$ найдутся такие различные определяюшие слова $R_{i}$ и $R_{j}$, что $K$ есть их максимальное общее начало. Заметим, что при этом в результате сокрашения подслова $K$ в слове $R_{i}^{-1} R_{j}$ получится непустое несократимое слово.

УСловиЕ $C(\lambda)$. Говорят, что симметризованное множество определяющих слов $\mathfrak{R}_{G}$ удовлетворяет условию малого сокращения $C(\lambda)$ (соответственно, $C^{\prime}(\lambda)$ ), где $\lambda$ - положительное действительное число, если для любого куска $K$ относительно множества $\mathfrak{R}_{G}$ и для любого определяюшего слова $R_{i}$ из этого множества равенство $R_{i}=K A$ влечет неравенство $|K|<\lambda\left|R_{i}\right|$ (соответственно, $\left.|K| \leqslant \lambda\left|R_{i}\right|\right)$. 
УСловиЕ $C_{m}$. Мы говорим, что множество определяюших слов $\Re$ удовлетворяет условию малого сокращения $C_{m}$, если оно симметризовано и никакое слово из $\mathfrak{R}$ не является произведением менее чем $m$ кусков относительно $\mathfrak{R}$.

Очевидно, условие $C(1 / m)$ влечет условие $C_{m+1}$.

Существенный вклад в развитие алгебраической теории малых сокрашений внес В. А. Тартаковский [98], [99]. Он решил проблему равенства слов для класса всех конечно определенных групп, удовлетворяюших условию $C(1 / 6)$. После него многие алгебраисты внесли свой вклад в развитие теории малых сокрашений (Дж. Бриттон, М. Гриндлингер, Р. Линдон, Г. С. Маканин и др.). М. Д. Гриндлингер [35], [36] показал, что к группам из этого класса применим алгоритм $M$. Дәна. Это означает, что любое слово, равное единище в группе из класса $C(1 / 6)$, содержит более половины циклической перестановки некоторого определяющего слова или ему обратного. Заменив в данном слове такое подслово $E$ определяющего слова $E Q$ равным ему дополнением $Q^{-1}$, мы получаем более короткое слово, равное исходному слову. В последовательном выполнении таких замен и состоит алгоритм Дэна. М. Гриндлингер также доказал разрешимость проблемы сопряженности для того же класса групп. В работе [53] Г. С. Маканин решил проблему равенства для групп с мерой наложения < 2/11. Наконец, Р. Линдон [46], используя так называемые диаграммы ван Кампена, доказал разрешимость проблемы равенства для класса всех конечно определенных групп, удовлетворяюших условию $C(1 / 5)$. Впоследствии выяснилось, что этот результат Р. Линдона уже неулучшаем (см. [34]). Заметим, что для групп из классов $C(2 / 11)$ и $C(1 / 5)$ алгоритм М. Дэна уже не работает. Соответствующий контрпример с мерой наложения $1 / 6$ был построен М. Гриндлингером в работе [35]. Он заметил, что в группе, заданной одним определяющим соотношением $a b c a^{-1} b^{-1} c^{-1}=1$, слово

$$
W \rightleftharpoons b^{-1} c^{-1} a c^{-1} a b a b c b c a^{-1} c a^{-1} b^{-1} a^{-1} b^{-1} c^{-1}
$$

равно 1 , хотя оно не содержит более половины ни одной циклической перестановки определяющего слова $a b c a^{-1} b^{-1} c^{-1}$ или ему обратного.

Следующая лемма хорошо известна и легко доказьвается индукцией по числу элементарных преобразований, переводящих слово $W$ в 1.

Лемма 5.1. Если $W$ - слово в алфавите группь $G$ с заданием (1), то $W=1$ в $G$ тогда и только тогда, когда в свободной группе при некоторых $U_{i}$ выполняется равенство

əде $\varepsilon_{i}= \pm 1$.

$$
W \equiv \prod_{i=1}^{k} U_{i} R_{j_{i}}^{\varepsilon_{i}} U_{i}^{-1}
$$

С каждым равенством $W=1$ в группе $G$ связьвается некоторая диаграмма ван Кампена, которая в определенном смысле заменяет последовательность элементарных преобразований $W \rightarrow \cdots \rightarrow 1$ рассматриваемой групшы. Эти диаграммы были впервые введены в работе [42]. В 1966 году Р. Линдон использовал их в своем доказательстве разрешимости проблемы равенства слов для класса всех конечно определенных групп, удовлетворяющих условию $C(1 / 5)$ или $C_{6}$.

Kapтой $M$ будем назьвать произвольньй конечньй набор попарно непересекаюшихся вершин, ребер и областей евклидовой плоскости, удовлетворяюший следуюшим условиям: 
(i) Каждое ребро е из $M$ гомеоморфно интервалу и имеет в $M$ две концевъе вериины $a$ и $b$, которые могут совпадать. Замыканием $\bar{e}$ ребра $е$ назьвается объединение $e \cup\{a, b\}$.

(ii) Каждая область $D$ из $M$ имеет связную топологическую границу $\partial(D)$, которая есть объединение замкнутых ребер

$$
\partial(D)=\overline{e_{1}} \cup \cdots \cup \overline{e_{k}} .
$$

Теоретико-множественное объединение вершин, ребер и областей данной карты $M$ будем обозначать через $\bar{M}$. Границу $\partial(\bar{M})$ множества $\bar{M}$ на плоскости $E^{2}$ будем назьвать также зраницей карты $M$. Ориентация на карте задается путем фиксирования ориентации на каждом ее ребре; при этом если $e$ - ориентированное ребро, идущее от его вершины $a$ к вершине $b$, то вершина $a$ называется начальной верииной этого ребра, а вершина $b$ - конечной. То же самое ребро $e$, ориентированное противоположным образом, назьвается обратным к ребру $е$ и обозначается через $e^{-1}$. Оно идет от вершиины $b$ к вершине $a$.

Путем в карте $M$ назьвается произвольная последовательность $L$ ее ориентированных замкнутых ребер $e_{1}, \ldots, e_{n}$ такая, что при любом $1 \leqslant i \leqslant n-1$ начальной вершиной ребра $e_{i+1}$ является конечная вершина ребра $e_{i}$. Началом пути $L$ называется начальная вершина ребра $e_{1}$, а концом - конечная вершина ребра $e_{n}$. Замкну-

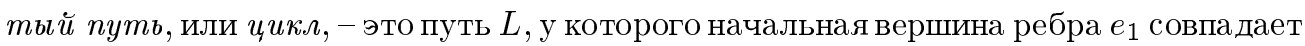
с конечной вершиной ребра $e_{n}$. Путь назьвается приведенным, если он не содержит пары рядом стоящих ребер вида $e$ и $e^{-1}$. Приведенньй путь $e_{1}, \ldots, e_{n}$ назьвается простым, если он не имеет самопересечений, т.е. если при всех $i \neq j$ начальные точки ребер $e_{i}$ и $e_{j}$ различны.

Граничнылм ииклом области $D$ назьвается любой цикл минимальной длины, включаюший в себя все ребра из границы $\partial(D)$ этой области, в котором все ребра ориентированы в соответствии с данной ориентацией области $D$. Если множество $\bar{M}$ связно и односвязно, то граничны.м ииклом карты $M$ называется цикл $L$ минимальной длины, содержаший все ребра из гранишы $\partial(\bar{M})$ и удовлетворяюший условию: любые два последовательных ребра $e_{i}$ и $e_{i+1}$ цикла $L$ с обшей вершиной $v$ являются соседними в циклическом упорядочении всех ребер карты $M$ при вершине $v$.

Диагр аммой над свободной группой $F$ называется ориентированная карта $M$ вместе с функцией $\varphi$, сопоставляющей каждому ориентированному ребру $e$ из карты $M$ метку в виде слова в алфавите группы $F$ таким образом, что $\varphi\left(e^{-1}\right)=(\varphi(e))^{-1}$.

Если $L=e_{1} \ldots e_{k}-$ произвольньй путь в карте $M$, то полагаем

$$
\varphi(L) \rightleftharpoons \varphi\left(e_{1}\right) \ldots \varphi\left(e_{k}\right)
$$

Меткой области $D$ из карты $M$ назьвается метка $\varphi(L)$ любого ее граничного цикла $L$. Заметим, что метка области определена неоднозначно и зависит как от выбора ориентации области, так и от выбора начала ее граничного цикла.

ЛЕмма 5.2. Пусть группа $G$ имеет представление (1). Для произвольной конечной последовательности $W_{1}, \ldots, W_{k}$ непустых несократимых слов свободной группь $F$ существует диаграмма $M\left(W_{1}, \ldots, W_{k}\right)$, удовлетворяющая следующим условиям:

(i) Eсли е - ребро из $M$, то $\varphi(e)$ есть непустое слово в алфавит группь $F$. 
(ii) Карта $\bar{M}$ связна и односвязна с некоторой выделенной веричной $O$ на границе $\partial(\bar{M})$. Существует граничньй цикл $e_{1} \ldots e_{k}$ карты $M$, начинающийся в вериине $O$ и такой, что произведение $\varphi\left(e_{1}\right) \ldots \varphi\left(e_{k}\right)$ несократимо $и$

$$
\varphi\left(e_{1}\right) \ldots \varphi\left(e_{k}\right)=W_{1} \ldots W_{k} \quad \text { в свободной əруппе. }
$$

(iii) Если $e_{1} \ldots e_{p}$ - граничный иикл произвольной области E карты $M$, то произведение $\varphi\left(e_{1}\right) \ldots \varphi\left(e_{p}\right)$ графически совпадает с некоторой перестановкой результата ииклического сокращения одного из слов $W_{i}$.

Такую диаграмму $M\left(W_{1}, \ldots, W_{k}\right)$ будем назьвать диаграммой последовательности $W_{1}, \ldots, W_{k}$.

ДоказАТЕльство. Доказательство проведем индукцией по $k$. Каждое несократимое слово $W_{i}$ однозначно представим в виде $U_{i} R_{i} U_{i}^{-1}$, где слово $R_{i}$ циклически несократимо.

Пусть $k=1$. Выберем некоторую вершину $v$ и при ней простой цикл $L$ длины $\left|R_{1}\right|$, ребра которого пометим последовательно буквами слова $R_{1}$. Если слово $U_{1}$ пусто, то искомая карта $M$ построена. В противном случае выберем некоторую вершину $O$ вне петли $е$ и простой путь из этой вершины в вершину $v$ длины $\left|U_{1}\right|$, ребра которого пометим буквами слова $U_{1}$.

При $k>1$ строим диаграммы $M_{1}, \ldots, M_{k}$ указанным образом для всех слов $W_{1}, \ldots, W_{k}$ и расположим их циклически в указанном порядке вокруг базисной точки $O$. Полученная диаграмма удовлетворяет всем условиям (i)-(iii), не считая отмеченного в (ii) условия несократимости метки граничного цикла. Это условие также будет выполнено, если произведение $\left(U_{1} R_{1} U_{1}^{-1}\right) \ldots\left(U_{k} R_{k} U_{k}^{-1}\right)$ было несократимо. В противном случае проведем индукцию по числу ребер в граничном цикле рассматриваемой карты путем отождествления соседних ребер, имеюших взаимно обратные метки. Очевидно, при этом остальные свойства построенной карты не будут нарушены. Лемма 5.2 доказана.

ЛЕмма 5.3. Если $W=\varphi(L)$ есть метка граничного иикла L некоторой связной односвязной диаграммы $M$ над свободной группой $F$, то можно указать такую нумерацию $D_{1}, \ldots, D_{m}$ всех областей карты $M$ и въбрать такие слова $U_{1}, \ldots, U_{m}$, длины которьх не больше суммы длин меток всех ориентированных ребер карты $M$, что в группе $F$ выполняется равенство

$$
W=\left(U_{1} R_{1} U_{1}^{-1}\right) \ldots\left(U_{m} R_{m} U_{m}^{-1}\right),
$$

где $R_{i}$ суть некоторые метки областей $D_{i}$.

ДоКАЗАТЕЛЬСТВо. Индукция по числу $m$ областей карты $M$. Если карта не содержит областей, то $L$ дерево и $\varphi(L)$ равно 1 в $F$.

Предположив, что доказываемое утверждение справедливо для карт с $m$ областями, докажем его для карты $M$ с $m+1$ областью.

Пусть $D$ - некоторая область карты $M$, имеющая общее ребро $е$ с ее границей. Обозначим через $M^{\prime}$ карту, полученную из $M$ удалением ребра $e$. Ясно, что полученная карта $M^{\prime}$ связна и односвязна и все области карты $M$, кроме области $D$, остаются областями карты $M^{\prime}$. Пусть $L=L_{1} e L_{2}$ и $e L_{3}$ - граничный цикл области $D$. Тогда 
$L^{\prime}=L_{1} L_{3}^{-1} L_{2}$-соответствуюший граничньй цикл карты $M^{\prime}$. Пусть $U$-метка ребра $e$ и $\varphi\left(L_{i}\right)=X_{i}$ при $i=1,2,3$. Тогда $W=\varphi(L)=X_{1} U X_{2}$ и $\varphi\left(L^{\prime}\right)=X_{1} X_{3}^{-1} X_{2}$.

По индуктивному предположению можно указать такую нумерацию $D_{1}, \ldots, D_{m}$ областей карты $M^{\prime}$ и выбрать такие слова $U_{1}, \ldots, U_{m}$, длины которых не больше суммы длин меток всех ориентированных ребер карты $M^{\prime}$, что при этом в группе $F$ выполняется равенство

$$
X_{1} X_{3}^{-1} X_{2}=\left(U_{1} R_{1} U_{1}^{-1}\right) \ldots\left(U_{m} R_{m} U_{m}^{-1}\right),
$$

где $R_{i}$ суть некоторые метки областей $D_{i}$.

Так как в свободной группе выполнено равенство

$$
W=X_{1} U X_{2}=\left(X_{1} X_{3}^{-1} X_{2}\right)\left(X_{2}^{-1} X_{3} U X_{2}\right),
$$

то для завершения доказательства достаточно положить $D_{m+1} \rightleftharpoons D, R_{m+1} \rightleftharpoons X_{3} U$ и $U_{m+1} \rightleftharpoons X_{2}^{-1}$. Очевидно, длины всех $U_{i}$ не превосходят суммы длин меток всех ребер карты $M$. Лемма 5.3 доказана.

ОПРЕДЕЛЕНИЕ. Пусть $\Re$ - симметризованное множество слов свободной группы $F$. Диаграмма $M$ назьвается $\Re$-диаграммой, если для любого граничного цикла $L$ любой области $D$ диаграммы $M \varphi(L) \in \Re$.

Из лемм 5.2 и 5.3 непосредственно следует

Теорема 5.1. Пусть $N$ - нормальная подгруппа, порожденная симметризованным множеством слов $\Re$ свободной группь $F, a W$ - произвольное несократимое непустое слово. Тогда $W \in N$ если и только если существует такая связная односвязная $\mathfrak{R}$-диаграмма $M$, что $W$ есть метка границы карты $M$.

Пусть $\mathfrak{R}$ - симметризованное множество слов свободной групшы $F$. Последовательность слов $W_{1}, \ldots, W_{m}$ называется $\Re$-последовательностью, если все слова $W_{i}$ сопряжены некоторым словам из $\mathfrak{R}$. $\mathfrak{R}$-последовательность $W_{1}, \ldots, W_{m}$ называется минимальной, если в свободной группе $F$ слово $W_{1} \ldots W_{m}$ не может быть записано в виде произведения менее чем $m$ слов, сопряженных словам из $\mathfrak{R}$.

Диаграмма $M$ над свободной группой $F$ назьвается приведенной, если для любых двух ее областей $D_{1}$ и $D_{2}$, имеюших общее ребро $е$ и граничные циклы $e L_{1}$ и $L_{2} e^{-1}$, метки $\varphi\left(L_{1}\right)$ и $\varphi\left(L_{2}\right)$ не являются взаимно обратньми словами.

ЛЕмма 5.4. Диаграмма $M$ любой минимальной $\Re$-последовательности является приведенной.

ДокАЗАТЕльСтво. Предположим, что некоторая минимальная $\mathfrak{R}$-последовательность $W_{1}, \ldots, W_{m}$ имеет неприведенную диаграмму $M$ с граничной меткой $W=W_{1} \ldots W_{m}$. Пусть $D_{1}$ и $D_{2}$ - области с общим ребром $e$, метки которых взаимно обратны. Очевидно, эти области $D_{1}$ и $D_{2}$ не могут совпадать. Объединим области $D_{1}$ и $D_{2}$ в одну область $D$, вычеркнув ребро $e$ и сохранив метки всех ребер. Тогда граничная метка объединенной области $D$ будет равна 1 в свободной группе. Полученная в результате указанного преобразования диаграмма $M^{\prime}$ будет связной и односвязной, причем диаграмма $M^{\prime}$ имеет ту же самую граничную метку $W$. Так как диаграмма $M^{\prime}$ имеет $m-1$ область, то по лемме $5.3 \mathrm{~W}$ равно в свободной групше 
произведению $m-1$ слов, каждое из которых сопряжено метке некоторой области из $M^{\prime}$. Все области из $M^{\prime}$, кроме области $D$, помечены элементами из $\mathfrak{R}$, а область $D$ помечена словом, равным 1 . В итоге получаем представление $W$ в виде произведения $m-2$ слов, каждое из которых сопряжено некоторому слову из $\mathfrak{R}$, что противоречит предположению о минимальности $\Re$-последовательности $W_{1}, \ldots, W_{m}$. Лемма 5.4 доказана.

Вершины и ребра карты $M$ называются граничнылми, если они лежат в $\partial(M)$. Область $D$ карты $M$ назьвается граничной областью, если ее граница имеет непустое пересечение с $\partial(M)$. В частности, это пересечение может состоять из одних вершин. Вершина, ребро или область карты $M$, не являюшиеся граничными, будем называть внутренними.

Для произвольной вершины $v$ карты $M$ через $d(v)$ обозначим ее $c m e n e н b$, т.е. число ребер с вершиной $v$. При этом если обе конщевые вершины ребра $е$ совпадают с вершиной $v$, то при подсчете степени $d(v)$ ребро $e$ считается дважды. Без ограничения обшности можно считать, что рассматриваемые диаграммы не имеют внутренних вершин степени 1.

Для произвольной области $D$ карты $M$ через $d(D)$ обозначается ее степень, т.е. число ребер в граничном цикле области $D$, а через $i(D)$ - число тех ребер в граничном цикле области $D$, которые не лежат на гранище карты $M$.

Можно предполагать, что в рассматриваемых диаграммах степень любой внутренней вершины $\geqslant 3$, так как метки двух внутренних ребер, имеюших обшую вершину степени 2, можно объединить в одну, удалив обшую вершину этих ребер.

Лемма 5.5. Пусть $M$ - приведенная $\Re$-диаграмма для симметризованного множсества $\mathfrak{R}$ слов свободной группь $F$. Если для множества слов $\mathfrak{R}$ выполняется условие малого сокращения $C_{6}$, то каждая область $D$, граница которой не содержст граничных ребер карты $M$, имеет степень $d(D) \geqslant 6$.

ДокаЗАТЕльство. Пусть $e$-внутреннее ребро карты $M$. Покажем, что его метка $W$ является куском.

Внутреннее ребро $е$ карты $M$ есть часть общей границы двух областей $D_{1}$ и $D_{2}$, которые имеют такие метки $R_{1}$ и $R_{2}$ из множества $\mathfrak{R}$, что $R_{1}=W A$ и $R_{2}=W^{-1} B$ при некоторых $A$ и $B$. Так как множество $\Re$ симметризовано и диаграмма $M$ является приведенной, то $A \neq B^{-1}$, т.е. $W$ является куском. Поэтому условие $C_{6}$ дает искомое неравенство $d(D) \geqslant 6$. Лемма 5.5 доказана.

Пусть $M$ - приведенная $\mathfrak{R}$-диаграмма для симметризованного множества $\mathfrak{R}$ слов свободной группы $F$, удовлетворяющего условию малого сокрашения $C_{6}$. Тогда каждая ее внутренняя область $D$ имеет степень $d(D) \geqslant 6$. По предположению в рассматриваемых картах степень любой внутренней вершины $\geqslant 3$, поэтому карта $M$ называется $(3,6)$-картой.

Карта, все внутренние вершины которой имеют степень не менее $p$, а все внутренние области имеют степень не менее $q$, назьвается $(p, q)$-картой. Карта, все внутренние вершины которой имеют степень не менее $p$, а все области имеют степень не менее $q$, называется $[p, q]$-картой.

Для каждой односвязной $(p, q)$-карты $M$ естественным образом строится двойсmвенная ей односвязная $[q, p]$-карта $M^{*}$. 
Областям $D_{j}$ карты $M$ взаимно однозначно соответствуют вершины $v_{j}^{*}$ карты $M^{*}$, причем $d\left(v_{j}^{*}\right)=i\left(D_{j}\right)$. Граничным областям карты $M$ соответствуют граничные вершины карты $M^{*}$. Внутренним ребрам карты $M$ взаимно однозначно соответствуют ребра $e^{*}$ карты $M^{*}$. Области $D^{*}$ карты $M^{*}$ взаимно однозначно соответствуют внутренним вершинам $v$ карты $M$, причем $d\left(D^{*}\right)=d(v)$.

Для построения карты $M^{*}$ в каждой области $D_{i}$ карты $M$ выбирается точка $v_{i}^{*}$, которая будет вершиной карты $M^{*}$. Если две различные области $D_{1}$ и $D_{2}$ имеют общее ребро $e$, то оно внутреннее. Тогда включаем в карту $M^{*}$ ребро $e^{*}$, идущее из вершины $v_{1}^{*}$ в вершину $v_{2}^{*}$ и пересекаюшее ребро $e$, но не пересекаюшее другие ребра карты $M$ и уже построенные ребра карты $M^{*}$. Если область $D_{i}$ лежит по обе стороны от некоторого ребра $e$ карты $M$, то в $M^{*}$ строим петлю с вершиной $v_{i}^{*}$, пересекаюшую ребро $e$, но не пересекаюшую другие ребра. Построенные таким образом вершины и ребра образуют скелетный граф $\Gamma^{*}$ искомой карты $M^{*}$. Области карты $M^{*}$ определим как области, ограниченные ребрами графа $\Gamma^{*}$. Каждая область $D^{*}$ содержит внутреннюю вершину карты $M$, из которой выходят все ребра карты $M$, пересекающие граничные ребра области $D^{*}$.

Если исходная карта $M$ была односвязной $(p, q)$-картой, то построенная таким образом двойственная карта $M^{*}$ будет односвязной $[q, p]$-картой.

Знак $\Sigma\left(\Sigma^{\cdot}, \Sigma^{\circ}\right)$ будем использовать для обозначения суммирования по всем вершинам или областям (соответственно, по всем граничныцм вершинам или областям, по всем внутренним вершинам или областям).

Для произвольной карты $M$ через $V_{M}$ обозначим число ее вершин, через $E_{M}-$ число ее неориентированны $x$ ребер, а через $F_{M}$ - число ее областей. Через $V_{M}$ будем обозначать число граничны $x$ вершин карты $M$, через $E_{M}^{\cdot}$ - число ее граничных ребер с учетом кратности, а через $F_{M}$ - число ее граничных областей.

ТЕОРема 5.2. Для произвольной односвязной карты $M$ выполняются равенcmsa

$$
6 Q_{M}=\Sigma^{\cdot}[6-d(v)]+\Sigma^{\circ}[6-d(v)]+2 \Sigma[3-d(D)]-2 E_{M}^{\cdot}
$$

$u$

$$
6 Q_{M}=\Sigma^{\cdot}[4-d(v)]+\Sigma^{\circ}[6-d(v)]+2 \Sigma[3-d(D)]+2\left(V_{M}^{*}-E_{M}^{*}\right),
$$

где $Q_{M}-$ число связных компонент карты $M$.

ДокАЗАТЕЛЬСтво. Обозначим через Г граф, образованньй вершинами и ребрами карты $M . \mathrm{K}$ каждой связной компоненте $\Gamma_{i}$ графа $\Gamma$ применим известную формулу Эйлера

$$
V_{i}-E_{i}+F_{i}=1,
$$

где $V_{i}$ - число вершин, $E_{i}$ - число ребер этого графа, а $F_{i}$ - число областей, на которые граф $\Gamma_{i}$ разбивает плоскость; при этом неограниченная область не учитывается. Просуммировав равенства (5) по всем связным компонентам графа $Г$, получим равенство

$$
V_{M}-E_{M}+F_{M}=Q_{M} .
$$


Очевидно, кроме того, выполнены равенства

$$
\begin{gathered}
\Sigma d(v)=2 E_{M}, \\
2 E_{M}=\Sigma d(D)+E_{M}^{\cdot} .
\end{gathered}
$$

Из предыдуших равенств получаем

$$
\begin{gathered}
6 V_{M}-6 E_{M}+6 F_{M}=6 Q_{M}, \\
6 E_{M}=\Sigma d(v)+2 \Sigma d(D)+2 E_{M}^{\cdot} .
\end{gathered}
$$

Последние равенства дают

$$
6 Q_{M}=6 V_{M}+6 F_{M}-\Sigma d(v)-2 \Sigma d(D)-2 E_{M}^{\cdot}
$$

откуда, объединив соответствующие слагаемые, получаем

$$
6 Q_{M}=\Sigma[6-d(v)]+2 \Sigma[3-d(D)]-2 E_{M}^{\cdot}
$$

Разбив суммирование по всем вершинам в (12) на два суммирования - по внутренним вершинам и по внешним, получим искомую формулу (3), а для получения формулы (4) остается расщепить первую сумму в формуле (3) на две суммы $\Sigma \cdot[4-d(v)]$ и $\Sigma \cdot 2=2 V_{M}$. Теорема 5.2 доказана.

СлЕДСТВИЕ 5.1. Если $M$ - односвязная $(3,6)$-карта, содержащая не менее двух областей, то

$$
\Sigma_{M}[4-i(D)] \geqslant 6
$$

ДокаЗАТЕльство. Рассмотрим двойственную карте $M$ карту $M^{*}$, которая, как было указано выше, является односвязной [6,3]-картой, содержашей не менее двух вершин, причем для каждой вершины $v^{*}$ карты $M^{*}$ имеем $d\left(v^{*}\right)=i(D)$, где $D-$ область, соответствуюшая этой вершине. Поэтому для доказательства неравенства (13) нам достаточно для односвязной [6,3]-карты $M^{*}$, содержащей не менее двух вершин, доказать неравенство

$$
\Sigma_{M}[4-d(v)] \geqslant 6 .
$$

Если все вершины карты $M^{*}$ изолированы, то неравенство (14) следует из условия, что число вершин не меньше двух. В случае, когда карта $M^{*}$ не имеет изолированных вершин, неравенство (14) следует из формулы (4), так как в ее правой части все слагаемые, кроме первого, неположительны. А общий случай легко сводится к двум указанньм переходом к подкарте карты $M^{*}$, получающейся вычеркиванием всех изолированных вершин. 
ЛЕмма 5.6. Если $M-$ односвязная [6,3]-карта, то

$$
\frac{1}{2} \Sigma_{M}^{\cdot}[6-d(v)] \geqslant E_{M}^{\cdot}
$$

$u$

$$
\frac{1}{2} \Sigma_{M}^{\cdot}[6-d(v)] \geqslant V_{M}^{\cdot}
$$

ДокАЗАТЕльство. Первое неравенство следует из формулы (3).

Если в карте $M$ нет изолированных вершин, то второе неравенство вытекает из первого, так как в таком случае имеем $V_{M}^{\cdot} \leqslant E_{M}^{\cdot}$. В противном случае обозначим через $M_{0}$ подкарту карты $M$, образованную изолированными вершинами. Для $M_{0}$ неравенство (15) очевидно. В общем случае (15) вытекает из соответствующих неравенств для карты $M_{0}$ и подкарты $M_{1}$ карты $M$, полученной удалением всех изолированных вершин, в силу равенства $V_{M}=V_{M_{1}}+V_{M_{0}}$.

ТЕОрема 5.3. Для произвольной односвязной $(3,6)$-карты $М$ выполняется неравенство

$$
F_{M} \leqslant \frac{1}{12}\left(\Sigma_{M}^{\cdot}[6-i(D)]\right)^{2}
$$

ДокаЗАТЕльство. Пусть $M$ - односвязная $(3,6)$-карта. Аналогично тому, как это мы делали выше, построим для $M$ двойственную односвязную $[6,3]$-карту $M^{*}$ и докажем для последней неравенство

$$
V_{M^{*}} \leqslant \frac{1}{12}\left(\Sigma_{M^{*}}^{\cdot}[6-d(v)]\right)^{2}
$$

из которого искомое неравенство (16) для $M$ вытекает непосредственно.

Для краткости будем опускать верхний индекс $*$ у $M^{*}$ и обозначать соответствуюшую сумму $\Sigma_{M}^{\cdot}[6-d(v)]$ через $\sigma(M)$. Тогда искомое неравенство (17) запишется в виде

$$
V_{M} \leqslant \frac{1}{12}(\sigma(M))^{2}
$$

Формула (3) дает нам для любой [6,3]-карты неравенство

$$
\sigma(M)=\Sigma_{M}^{\cdot}[6-d(v)] \geqslant 6 .
$$

Поэтому добавление изолированной вершины в карту усиливает неравенство (18). Следовательно, можем считать, что в $M$ нет изолированных вершин.

Если все вершины $[6,3]$-карты $M$ являются граничньми, то в силу (15) имеем

$$
V_{M}=V_{\dot{M}}^{\cdot} \leqslant \frac{1}{2} \Sigma_{M}^{\cdot}[6-d(v)]
$$

откуда в силу неравенства (19) следует неравенство (18) в случае отсутствия внутренних вершин. 
В случае наличия внутренних вершин сначала удалим из $M$ все компоненты, являющиеся деревьями, и все деревья, прикрепленные к карте $M$. Для этого достаточно убедиться, что добавление в [6,3]-карту одной новой граничной вершины степени 1 также усиливает неравенство (18).

В самом деле, пусть карта $M^{\prime}$ получается из карты $M$ удалением одной граничной вершины $v$ степени 1 и ребра $e$, имеюшего $v$ своей вершиной. Вклад вершины $v$ в сумму $\sigma(M)$ равен 5. В результате удаления вершины $v$ степень $d(u)$ другой вершины $u$ ребра $е$ уменьшится на 1 , но она остается граничной вершиной для $M^{\prime}$. Так что $M^{\prime}$ есть односвязная [6,3]-карта. Следовательно, удаление одной граничной вершшны $v$ степени 1 приводит к новой односвязной [6,3]-карте, уменьшая значение $\sigma(M)$ на 4. Поэтому в силу (19) при переходе от $M^{\prime}$ к $M$ неравенство (18) усилится. Нам остается повторить удаление вершин степени 1 до тех пор, пока не исчезнут все деревья в $M$.

Если исходная [6,3]-карта $M$ имела внутренние вершины, то полученная карта $M^{\prime}$ без изолированных вершин и граничных вершин степени 1 будет непустой. Без ограничения обшности можно считать, что такой уже была исходная [6,3]-карта $M$.

Доказательство неравенства (18) для таких [6, 3]-карт проведем индукцией по числу вершин.

Обозначим через $M_{1}$ карту, получаюшуюся из карты $M$ удалением всех ее граничных вершин, граничных ребер и граничных областей. Тогда $V_{M}=V_{M_{1}}+V_{\dot{M}}$.

Если $v \in M_{1}$, то $d_{M}(v) \geqslant 6$, так как $v$ - внутренняя вершина карты $M$. Степень $d_{M}(u)$ каждой граничной вершины $u$ карты $M_{1}$ складывается из ребер, входящих в степень $d_{M_{1}}(u)$ в карте $M_{1}$, плюс число выходящих из нее ребер, второй конец которых попал в $\partial(M)$. Так как в карте $M$ нет изолированных вершин и граничных вершин степени 1 , то число ребер, оба конца которых попали в $\partial(M)$, не меншше чем $V_{M}^{*}$. Следовательно, справедливо неравенство

$$
\Sigma_{M}^{\cdot} d_{M}(v)-\Sigma_{M_{1}}^{\cdot}\left[d_{M}(v)-d_{M_{1}}(v)\right] \geqslant 2 V_{M}^{\cdot},
$$

из которого в силу (14) следует

$$
\begin{aligned}
\sigma(M)-\sigma\left(M_{1}\right) & =6 V_{M}^{\cdot}-\Sigma_{M}^{\cdot} d_{M}(v)-\Sigma_{M_{1}}^{\cdot}\left[6-d_{M_{1}}(v)\right] \\
& \geqslant 6 V_{M}^{\cdot}-\Sigma_{M}^{\cdot} d_{M}(v)-\Sigma_{M_{1}}^{\cdot}\left[d(v)-d_{1}(v)\right] \\
& \geqslant 2 \Sigma_{M}^{\cdot}[4-d(v)] \geqslant 12,
\end{aligned}
$$

т.е. имеем неравенство

$$
\sigma(M)-\sigma\left(M_{1}\right) \geqslant 12 .
$$

Так как $V_{M}=V_{M_{1}}+V_{\dot{M}}$ и в рассматриваемом случае карта $M$ содержит внутреннюю вершину, то, используя индуктивное предположение (18) для карты $M_{1}$ вместе с соотношением (15) для $M$ и неравенством (21), получим неравенства

$$
\begin{aligned}
V_{M} & \leqslant \frac{1}{12} \sigma\left(M_{1}\right)^{2}+\frac{1}{2} \sigma(M) \leqslant \frac{1}{12}(\sigma(M)-12)^{2}+\frac{1}{2} \sigma(M) \\
& =\frac{1}{12}\left[\sigma(M)^{2}-18 \sigma(M)+144\right] .
\end{aligned}
$$

Из (19) для $M_{1}$ и $(21)$ следует $\sigma(M) \geqslant 12$. Поэтому имеем

$$
V_{M}<\frac{1}{12} \sigma(M)^{2},
$$

т.е. искомое неравенство (18) доказано. 
ТеоРема 5.4 (Р. Линдон). Проблема равенства разрешима в классе всех конечно определенных групп, которье имеют задание, удовлетворяющее условию малого сокращения $C_{6}$.

ДокАЗАТЕльСтво. Пусть группа $G$ задана симметризованным множеством определяюших слов $\mathfrak{R}$ в алфавите свободной группы $F$. Допустим, что слово $W$ равно 1 в группе $G$. Тогда диаграмма $M$ минимальной $\Re$-последовательности для $W$ будет $(3,6)$-картой. По теореме 5.3 вьполняется неравенство

$$
F_{M} \leqslant \frac{1}{12}\left(\Sigma_{M}^{\cdot}[6-i(D)]\right)^{2} .
$$

Каждая область $D$, все граничные ребра которой внутренние в $M$, по лемме 5.5 имеет степень $i(D)=d(D) \geqslant 6$. Так как при этом граничные ребра карты $M$ могут содержать не более чем $|W|$ областей, то из неравенства (13) получаем

$$
0<\Sigma_{M}^{\cdot}[6-i(D)] \leqslant 6|W|,
$$

откуда в силу (22) следует $F_{M} \leqslant 3|W|^{2}$.

Пусть

$$
L \rightleftharpoons \max _{R_{i} \in \mathfrak{R}}\left|R_{i}\right| .
$$

По лемме 5.3 найдутся такие слова $R_{i}$ из множества $\mathfrak{R}(i=1, \ldots, m)$, где $m \leqslant 3|W|^{2}$, и такие слова $U_{i}$ длины, не превосходяшей числа $3|W|^{2} L+|W|$, что

$$
W \equiv\left(U_{1} R_{1} U_{1}^{-1}\right) \ldots\left(U_{m} R_{m} U_{m}^{-1}\right) .
$$

Таким образом, алгоритм распознавания равенства $W=1$ в $G$ сводится к проверке равенства $W=X$ в свободной группе для слов $X$ указанного в (23) вида, длина которых ограничена сверху числом $6|W|^{2}\left(3|W|^{2} L+|W|+L\right)$.

Из доказанной теоремы непосредственно следует

СлЕДСТвИЕ 5.4.1. Проблема равенства разрешима в классе всех конечно определенных групп, которые имеют задание, удовлетворяющее условию малого сокращения $C(1 / 5)$.

Примеры групп с неразрешимой проблемой равенства возникают уже в классе $C^{\prime}(1 / 5)$, отличаюшемся от $C(1 / 5)$ заменой в определении строгого неравенства на нестрогое. Это следует из приводимого ниже любопытного утверждения А.И. Гольберга [34] и теоремы 6.5 .

Лемма 5.7. Каждая конечно определенная группа может быть задана c помощью системы определяющих соотношений, удовлетворяющей условию $C^{\prime}(1 / 5)$. 
ДокАЗАТЕльство. Сначала докажем, что тривиальная группа имеет следующее задание в 4-буквенном алфавите:

$$
H=\left\langle\left\langle a, b, c, d \mid a^{6}=1, b^{5}=1, c^{5}=1, b^{-1} a b c d=1, d^{-1} b d^{2} c=1, c a c a^{-1} b^{-1}=1\right\rangle\right\rangle .
$$

В самом деле, из равенств $d^{-1} b d^{2} c=1$ и $b^{-1} a b c d=1$ следует сопряженность элементов $a$ и $b$, что в силу равенств $a^{6}=1, b^{5}=1$ дает $a=b=1$. Далее, используя равенства $c a c a^{-1} b^{-1}=1$ и $c^{5}=1$, получаем $c=1$, а значит, и $d=1$.

Пусть конечно определенная группа $G$ имеет задание

$$
\left\langle\left\langle a_{1}, \ldots, a_{n} \mid R_{1}=1, \ldots, R_{m}=1\right\rangle\right\rangle .
$$

Добавим к алфавиту группы $G$ новые буквы $x_{1}, \ldots, x_{4 m}$, где $4 m \geqslant \Sigma_{i=1}^{m}\left|R_{i}\right|$. Множество определяюших соотношений искомого задания группы $G$ состоит из $m$ шестерок соотношений, копируюших задание групшы $H$ в 4-буквенном алфавите $x_{4 i+1}, x_{4 i+2}$, $x_{4 i+3}, x_{4 i+4}$, к которым добавляется по одному соотношению вида $\overline{R_{i}}=1$ для каждого соотношения $R_{i}=1$ групшы $G$. Именно, если $R_{j}=a_{i_{1}} a_{i_{2}} \ldots a_{i_{t_{j}}}=1$, то в качестве искомого слова $\overline{R_{j}}$ берем слово

$$
a_{i_{1}} y_{1} a_{i_{2}} y_{2} \ldots a_{i_{j}} y_{t_{j}}
$$

где буквы $y_{s}$ подбираются из нашего запаса $x$-букв так, чтобы каждая из них встречалась не более одного раза и только в одном из слов (24). Легко убедиться, что полученное задание группы $G$ удовлетворяет условию $C^{\prime}(1 / 5)$.

\section{6. Неразрешимость проблемы равенства для групп}

Сначала приведем предложенную П. С. Новиковым в работе [72] технику правильных систем проходных букв, на которой было основано его доказательство теоремы о неразрешимости проблемы равенства для конечно определенных групп. В случае, когда правильная система проходных букв состоит из одной буквы, получается понятие, которое за несколько лет до работы П. С. Новикова было введено в совместной работе трех авторов [40] и впоследствии получило название "HNN-расширение" (HNN-extension).

Пусть дана конечно определенная группа

$$
G=\left\langle\left\langle a_{1}, \ldots, a_{n} \mid R_{1}=1, \ldots, R_{m}=1\right\rangle\right\rangle .
$$

Добавим к алфавиту группы $G$ новую букву $t$, а к ее определяюшим соотношениям добавим конечное число соотношений вида

$$
A_{j} t=t B_{j} \quad(j=1, \ldots, k)
$$

где $A_{j}, B_{j}$ - слова в алфавите групшы $G$. Полученную группу обозначим через $G^{(t)}$.

Пусть коэффициенты $A_{1}, \ldots, A_{k}$ и $B_{1}, \ldots, B_{k}$ порождают в группе $G$ подгруппы $H_{1}$ и $H_{2}$. Буква $t$ называется правильной проходной буквой группь $G^{(t)}$ с базой $G$ (при этом группа $G^{(t)}$ назьвается HNN-расширением группы $G$ ), если отображение $\varphi_{t}$, заданное равенствами $\varphi_{t}\left(A_{j}\right)=B_{j}(j=1, \ldots, k)$, порождает изоморфное отображение 
подгруппы $H_{1}$ на подгруппу $H_{2}$. Очевидно, это отображение будет изоморфизмом в том и только том случае, если для любого слова $f\left(A_{1}, \ldots, A_{k}\right)$, т.е. произведения, составленного из множителей вида $A_{i}^{ \pm 1}$, имеем:

$$
f\left(A_{1}, \ldots, A_{k}\right)=1 \text { в группе } G \Longleftrightarrow f\left(B_{1}, \ldots, B_{k}\right)=1 \text { в группе } G \text {. }
$$

Заметим, что если все соотношения (1) групшы $G^{(t)}$ имеют вид $A t=t A$, где $t$ не входит в слова $A$, то буква $t$ очевидным образом является правильной проходной буквой.

Произвольная правильная система проходных букв по П. С. Новикову получается простым объединением конечного числа правильных проходных букв с одной и той же базой $G$ и объединением соответствуюших определяющих соотношений вида (1).

Рассмотрим группу $G^{(t)}$, полученную из группы $G$ добавлением правильной проходной буквы $t$. Буквы $t^{ \pm 1}$ входят только в соотношения (1) и в тривиальные соотношения $t t^{-1}=1$ и $t^{-1} t=1$. Можно считать, что элементарные преобразования, вьполняемые в группе $G^{(t)}$ на основе определяюших соотношений $(1)$, имеют вид

$$
P \underline{t} Q \rightarrow P A_{j} \underline{\underline{t}} B_{j}^{-1} Q \text { или } P \underline{t} Q \rightarrow P A_{j}^{-1} \underline{t} B_{j} Q .
$$

В преобразованиях вида (2) мы будем считать, что выделенная в левой части буква $\underline{t}$ не затрагивается и переходит по индивидуальности в букву $\underline{t}$, выделенную в правой части. Считая, что все вхождения букв $t$ и $t^{-1}$ в слова $P$ и $Q$ также сохраняют свою индивидуальность, мы распространим это отношение совпадения по индивидуальности вхождений проходной буквы $t$ на любую последовательность элементарных преобразований. Заметим, что в произвольной последовательности элементарных преобразований каждое вхождение буквы $t^{ \pm 1}$ в преобразуемое слово $X_{i}$ совпадает по индивидуальности либо с некоторой буквой $t^{ \pm 1}$, входяшей в начальное слово рассматриваемой последовательности, либо с некоторой буквой $t^{ \pm 1}$, вставленной в этой последовательности до момента появления слова $X_{i}$ включительно.

Договоримся обозначать через $u$ (или $u$ с некоторым индексом) произвольное слово вида $f\left(A_{1}, \ldots, A_{k}\right)$, а через $v$ (или $v$ с индексом) - соответствующее слово вида $v_{j}=f^{-1}\left(B_{1}, \ldots, B_{k}\right)$, так что $v_{j}^{-1}-$ это образ $u_{j}$ при отображении $\varphi_{t}$. Очевидно, для любых слов $u$ и $v$ в алфавите групшы $G \varphi_{t}(u)=v^{-1}$ тогда и только тогда, когда имеется последовательность элементарных преобразований

$$
t \rightarrow \cdots \rightarrow u t v
$$

Последовательность элементарных преобразований (3) будем записывать в виде $t \rightarrow u t v$ и называть составным преобразованием для буквы $t$. Аналогично имеем составные преобразования для буквы $t^{-1}: t^{-1} \rightarrow v t^{-1} u$.

Мы докажем, что если отображение $\varphi_{t}$ является изоморфизмом, то группа $G^{(t)}$ есть расширение групшы $G$. Именно поэтому используется термин HNN-расширение групшы $G$.

Следующая лемма очевидна.

ЛЕмма 6.1. Если в последовательности әлементарных преобразований без вставок букв $t$

$$
X_{1} t Y_{1} \rightarrow X_{2} t Y_{2} \rightarrow \cdots \rightarrow X_{s} t Y_{s}
$$


все выделенные вхождения буквы $t$ совпадают по индивидуальности, то можно указать такие слова и и $v$, что $\varphi_{t}(u)=v^{-1}$ (m.е. для них существует последовательность (3) из преобразований вида (2)), и последовательности без вставок буквы $t$ :

$$
X_{1} u \rightarrow \cdots \rightarrow X_{s} \quad u \quad v Y_{1} \rightarrow \cdots \rightarrow Y_{s} .
$$

Аналогично для последовательности әлементарных преобразований без вставок $t$

$$
X_{1} t^{-1} Y_{1} \rightarrow X_{2} t^{-1} Y_{2} \rightarrow \cdots \rightarrow X_{s} t^{-1} Y_{s}
$$

где все выделенные вхождения буквы $t^{-1}$ совпадают по индивидуальности, можно указать такие слова $u_{1} u v_{1}$, что $\varphi_{t}\left(u_{1}\right)=v_{1}^{-1}$, и последовательности әлементарных преобразований без вставок буквы $t$ :

$$
X_{1} v_{1} \rightarrow \cdots \rightarrow X_{s} \quad u \quad u_{1} Y_{1} \rightarrow \cdots \rightarrow Y_{s}
$$

ОСНовная ЛЕммА. Пусть $t-$ правильная проходная буква в группе $G^{(t)}$ и в слово $Y$ не входит буква $t^{ \pm 1}$. Если $X=Y$ в группе $G^{(t)}$, то найдется последовательность элементарных преобразований

$$
X=X_{1} \rightarrow X_{2} \rightarrow \cdots \rightarrow X_{s}=Y
$$

не содержащая вставок буквы $t$.

ДокАЗАТЕЛЬСТво. Для доказательства мы добавим к элементарным и составным преобразованиям (2) и (3) составные преобразования вида

$$
P t^{-1} Q \rightarrow P v t^{-1} u Q
$$

где $\varphi_{t}(u)=v^{-1}$

По условию имеем некоторую последовательность (5) элементарных преобразований. Наша задача исключить в ней вставки буквы $t$. Мы это сделаем индукцией по числу вставок буквы $t$ в последовательности (5).

Выделим в последовательности (5) последнюю вставку буквы $t$. Без ограничения обшности можно считать, что эта вставка левосторонняя, т.е. последовательность (5) имеет вид

(6) $X \mp X_{1} \rightarrow X_{2} \rightarrow \cdots \rightarrow X_{r}=P_{1} Q_{1} \rightarrow P_{1} \underline{t} \underline{t}^{-1} Q_{1}=X_{r+1} \rightarrow \cdots \rightarrow X_{s}=Y$.

Так как буквы $t^{ \pm 1}$ в слово $Y$ не входят, то вставленные буквы $\underline{t}$ и $\underline{t}^{-1}$ должны сократиться. Возможны три случая.

1) Вставленные буквы $\underline{t}$ и $\underline{t}^{-1}$ сокрашаются одновременно.

2) Буква $\underline{t}$ сокращается раньше с некоторой буквой $t^{-1}$ из слова $P_{1}$.

3) Буква $\underline{t}^{-1}$ сокрашается раншше с некоторой буквой $t$ из слова $Q_{1}$.

Рассмотрим случай 1). Выделим в последовательности (6) момент взаимного сокращения букв $\underline{t}$ и $\underline{t}^{-1}$ :

$$
\begin{aligned}
X \mp X_{1} & \rightarrow X_{2} \rightarrow \cdots \rightarrow X_{r} \mp P_{1} Q_{1} \rightarrow P_{1} \underline{t}^{-1} Q_{1} \mp X_{r+1} \\
& \rightarrow \cdots \rightarrow X_{r+h} \mp P_{h} \underline{t}^{-1} Q_{h} \rightarrow X_{r+h+1} \mp P_{h} Q_{h} \rightarrow \cdots \rightarrow X_{s} \mp Y .
\end{aligned}
$$


В последовательности $X_{r+1} \rightarrow \cdots \rightarrow X_{r+h}$ нет вставок буквы $t$ и выделенная буква $\underline{t}$ сохраняет индивидуальность. В силу леммы 6.1 найдутся такие $u_{1}$ и $v_{1}$, что $\varphi_{t}\left(u_{1}\right)=v_{1}^{-1}$ и имеются последовательности элементарных преобразований без вставок буквы $t$

$$
P_{1} u_{1} \rightarrow \cdots \rightarrow P_{h}
$$

и

$$
v_{1} \underline{t}^{-1} Q_{1} \rightarrow \cdots \rightarrow \underline{t}^{-1} Q_{h}
$$

Так как выделенные в последовательности (9) буквы $\underline{t}^{-1}$ совпадают по индивидуальности, то по лемме 6.1 найдутся такие $u_{2}$ и $v_{2}$, что $\varphi_{t}\left(u_{2}\right)=v_{2}^{-1}$ и имеются последовательности элементарных преобразований без вставок буквы $t$

$$
v_{1} v_{2} \rightarrow \cdots \rightarrow 1
$$

и

$$
u_{2} Q_{1} \rightarrow \cdots \rightarrow Q_{h}
$$

Из $(10)$, используя изоморфизм $\varphi_{t}$, получаем в группе $G$ равенство $u_{1} u_{2}=1$, значит, существует последовательность элементарных преобразований группы $G$

$$
1 \rightarrow \cdots \rightarrow u_{1} u_{2}
$$

Теперь, используя начало и конец последовательности (7) и последовательности $(12),(8)$ и $(11)$, в которых нет вставок буквы $t$, получим новую последовательность

$$
\begin{aligned}
X=X_{1} \rightarrow \stackrel{(7)}{\rightarrow} \rightarrow X_{r}=P_{1} Q_{1} \rightarrow \stackrel{(12)}{\rightarrow} \rightarrow P_{1} u_{1} u_{2} Q_{1} \\
\quad \rightarrow \stackrel{(8)}{\rightarrow} \rightarrow P_{h} u_{2} Q_{1} \rightarrow \stackrel{(11)}{\rightarrow} \rightarrow P_{h} Q_{h}=X_{r+h} \rightarrow \stackrel{(7)}{\rightarrow} \rightarrow X_{s}=Y,
\end{aligned}
$$

в которой исключена последняя вставка буквы $t$ из (7).

Случай 2). Выделим в $P_{1}$ букву $t^{-1}$, которая должна сократиться с буквой $\underline{t}$, а в $(6)$ - момент сокрашения с ней нашей буквы $\underline{t}$ :

$$
\begin{aligned}
X \mp X_{1} \rightarrow \ldots X_{r}= & P_{1} Q_{1}=T_{1} t^{-1} K_{1} Q_{1} \rightarrow T_{1} t^{-1} K_{1} \underline{t} \underline{t}^{-1} Q_{1}=X_{r+1} \\
& \rightarrow \cdots \rightarrow X_{r+h}=T_{h} t^{-1} \underline{t} Q_{h} \rightarrow T_{h} Q_{h} \rightarrow \cdots \rightarrow X_{s}=Y .
\end{aligned}
$$

Как и в предыдущем случае, в последовательности

$$
X_{r+1} \rightarrow \cdots \rightarrow X_{r+h}
$$

нет вставок $t$ и выделенные буквы $t^{-1}$ и $\underline{t}$ во всех словах имеют одну и ту же индивидуальность. В силу леммы 6.1 , примененной к левой букве $t^{-1}$, найдем при некоторых $u_{1}, v_{1}\left(\varphi_{t}\left(u_{1}\right)=v_{1}^{-1}\right)$ следующие последовательности без вставок $t$ :

$$
\begin{gathered}
T_{1} v_{1} \rightarrow \cdots \rightarrow T_{h}, \\
u_{1} K_{1} \underline{t}^{-1} Q_{1} \rightarrow \cdots \rightarrow \underline{t} Q_{h} .
\end{gathered}
$$


Применив лемму 6.1 к букве $\underline{t}$ в последовательности (15), получим при некоторых $u_{2}, v_{2}\left(\varphi_{t}\left(u_{2}\right)=v_{2}^{-1}\right)$ последовательности без вставок $t$

$$
\begin{gathered}
u_{1} K_{1} u_{2} \rightarrow \cdots \rightarrow 1, \\
v_{2} t^{-1} Q_{1} \rightarrow \cdots \rightarrow Q_{h} .
\end{gathered}
$$

При этом мы имеем следуюшие составные преобразования:

$$
\begin{aligned}
t^{-1} & \rightarrow v_{1} t^{-1} u_{1}, \\
t & \rightarrow u_{2} t v_{2} .
\end{aligned}
$$

Наряду с (19) мы имеем для $t^{-1}$ составное преобразование

$$
t^{-1} \rightarrow v_{2} t^{-1} u_{2}
$$

Теперь, используя начало и конец последовательности (13), а также последовательности элементарных преобразований без вставок $t$, получим новую последовательность, которая содержит на одну вставку меньше, чем было в (13):

$$
\begin{aligned}
X & =X_{1} \rightarrow \stackrel{(13)}{\cdots} \rightarrow X_{r}=P_{1} Q_{1}=T_{1} t^{-1} K_{1} Q_{1} \rightarrow^{(18)(20)} \rightarrow T_{1} v_{1} v_{2} t^{-1} u_{2} u_{1} K_{1} Q_{1} \\
& \rightarrow \stackrel{(16)}{\rightarrow} \rightarrow T_{1} v_{1} v_{2} t^{-1} Q_{1} \rightarrow \stackrel{(14)}{\rightarrow} \rightarrow T_{h} v_{2} t^{-1} Q_{1} \rightarrow \stackrel{(17)}{\rightarrow} \rightarrow T_{h} Q_{h} \rightarrow \stackrel{(13)}{\rightarrow} \rightarrow X_{s}=Y .
\end{aligned}
$$

Случай 3 ) симметричен случаю 2). Доказательство основной леммы завершено.

Из основной леммы непосредственно вытекает следуюшая теорема.

ТЕОРема 6.1. Если $t$ - правильная проходная буква группы $G^{(t)}$, то для любых слов $X$ и $Ү$ в алфавите группы $G$ имеем

$$
X=Y \text { в гpynne } G \Longleftrightarrow X=Y \text { в гpynne } G^{(t)},
$$

m.e. $G$ есть подгруппа групnь $G^{(t)}$.

Еще одно следствие из доказанной основной леммы носит в литературе название "лемма Бриттона". Оно неоднократно доказьвалось мимоходом в работе П. С. Новикова [72], но не было там явно выделено. Дж. Бриттон сформулировал ее явно в работе [27].

Лемма Бриттона. Пусть $G^{(t)}$ есть HNN-расширение группь $G$. Если слово $X$ содержит букву $t$ или $t^{-1}$ и $X=1$ в группе $G^{(t)}$, то $X$ имеет вид $X=$ $P t^{\sigma} E t^{-\sigma} Q$, где $\sigma= \pm 1, E \in H_{1}$ при $\sigma=-1$ u $E \in H_{2}$ при $\sigma=1$. 
ДОкАЗАТЕЛЬСтвО. Пусть $X=1$ в групше $G^{(t)}$. По основной лемме найдется последовательность элементарных преобразований без вставок $t$ :

$$
X \rightarrow \cdots \rightarrow 1 .
$$

Все входяшие в $X$ буквы $t^{ \pm 1}$ должны сократиться между собой. Выделим в $X$ те буквы $t$ и $t^{-1}$, которые в последовательности (21) сокрашаются первыми. Очевидно, слово $X$ имеет вид $X=P t^{\sigma} E t^{-\sigma} Q$, где $\sigma= \pm 1$, и $E$ не содержит $t^{ \pm 1}$. Тогда последовательность (21) имеет вид

$$
X=P \underline{t}^{\sigma} E \underline{t}^{-\sigma} Q \rightarrow \cdots \rightarrow P_{h} \underline{t}^{\sigma} \underline{t}^{-\sigma} Q_{h} \rightarrow P_{h} Q_{h} \rightarrow \cdots \rightarrow 1 .
$$

Пусть $\sigma=1$. Применяя лемму 6.1, найдем такие $u_{1}, v_{1}$ и $u_{2}, v_{2}$, что $\varphi_{t}\left(u_{i}\right)=v_{i}^{-1}$, причем имеем последовательности преобразований без вставок $t$

$$
\begin{aligned}
& P u_{1} \rightarrow \cdots \rightarrow P_{h}, \\
& v_{1} E v_{2} \rightarrow \cdots \rightarrow 1, \\
& u_{2} Q \rightarrow \cdots \rightarrow Q_{h} .
\end{aligned}
$$

Так как $E$ не содержит букв $t^{ \pm 1}$, то из (22) получаем $E=v_{1}^{-1} v_{2}^{-1}$ в $G$, т.е. $E \in H_{2}$. В случае $\sigma=-1$ аналогично получаем $E \in H_{1}$. Лемма доказана.

ОпРЕДЕлЕниЕ. Пусть $t$ - правильная проходная буква группы $G^{(t)}$ относительно группы $G$. Тогда разложение слова $X$ :

$$
X=E_{0} t^{\sigma_{1}} E_{1} t^{\sigma_{2}} \ldots E_{n-1} t^{\sigma_{n}} E_{n},
$$

где выделены все вхождения букв $t^{ \pm 1}$, называется приведенной формой, если в него не входят подслова вида $t^{\sigma} E_{i} t^{-\sigma}$, где $\sigma=1$ и $E_{i} \in H_{2}$ или $\sigma=-1$ и $E_{i} \in H_{1}$.

Очевидно, что для любого слова $X$ существует равное ему слово, которое записано в приведенной форме.

Следуюшие три теоремы легко вытекают из леммы Бриттона.

Tеорема 6.2. Пусть $t$ - правильная проходная буква в группе $G^{(t)}$ с основанием $G$ и

$$
X=E_{0} t^{\sigma_{1}} E_{1} t^{\sigma_{2}} \ldots E_{n-1} t^{\sigma_{n}} E_{n}
$$

- приведенная форма слова $X$.

Если $n \geqslant 1$, то слово X не равно единице в группе $G^{(t)}$.

$\Pi р и ~ n=0$ слово $X$ равно единице в группе $G^{(t)}$ тогда и только тогда, когда $X$ равно единиче в исходной группе $G$.

TeOPEMA 6.3. Пусть гpynna

$$
G^{(t)}=\left\langle\left\langle a_{1}, \ldots, a_{n}, t \mid R_{1}=1, \ldots, R_{m}=1, A_{1} t=t B_{1}, \ldots, A_{k} t=t B_{k}\right\rangle\right\rangle
$$

является $H N N$-расиирением конечно определенной группы $G$. Eсли $G$ имеет разрешимую проблему равенства и разрешимые проблемы вхождения в подгруппь $H_{1}$ и $H_{2}$, порожденные, соответственно, словами $A_{1}, \ldots, A_{k}$ и $B_{1}, \ldots, B_{k}$, то в группе $G^{(t)}$ также разрешима проблема равенства. 
Теорема 6.4. Пусть группа $G^{(t)}$ является $H N N$-расширением группь G. Тогда каждый әлемент конечного порядка в $G^{(t)}$ сопряжен с некоторым әлементом конечного порядка группы $G$. Группа $G^{(t)}$ не имеет кручения тогда и только тогда, когда не имеет кручения исходная группа $G$.

Теорема 6.5 (П.С. Новиков [72]). Существует конечно определенная группа $c$ неразрешимой проблемой равенства слов.

ДокАЗАТЕльство. Вскоре после опубликования работы П. С. Новикова [72] появились более простые конструкции групп с неразрешимой проблемой равенства слов. Мыприведем здесь упрошенное доказательство теоремы П. С. Новикова, отражающее вклад В. В. Буна [21], [22], Дж. Бриттона [26] и В. В. Борисова [25].

Пусть

$$
\Pi=\left\langle a_{1}, a_{2}, \ldots, a_{n} \mid E_{1}=F_{1}, \ldots, E_{m}=F_{m}\right\rangle
$$

есть задание полугруппы с неразрешимой проблемой равенства $X=q$ для фиксированного слова $q$, причем, все слова $E_{i}$ и $F_{i}(i=1, \ldots, m)$ непустые.

Рассмотрим группу $G$, которая задается образуюшими

$$
a_{1}, a_{2}, \ldots, a_{n}, l, r, c, t, k
$$

и следуюшими определяющими соотношениями:

(i) $a_{j} l=l^{m+1} a_{j}(1 \leqslant j \leqslant n), r a_{j}=a_{j} r^{m+1}(1 \leqslant j \leqslant n)$,

(ii) $c a_{j}=a_{j} c(1 \leqslant j \leqslant n),\left(l^{i} E_{i} r^{i}\right) c=c\left(l^{i} F_{i} r^{i}\right)(1 \leqslant i \leqslant m)$,

(iii) $c t=t c, l t=t l$,

(iv) $c k=k c, r k=k r,\left(q^{-1} t q\right) k=k\left(q^{-1} t q\right)$.

Буква $k$ в группе $G$ образует правильную систему проходных букв, так как она участвует только в определяюших соотношениях (iv), и группа $G_{1}$, заданная образуюшими $a_{1}, a_{2}, \ldots, a_{n}, l, r, c, t$ и определяюшими соотношениями (i)-(iii), есть базис групшы $G$ по этой системе.

В группе $G_{1}$ буква $t$, участвующая только в определяюших соотношениях (iii) группы $G_{1}$, образует правильную систему проходных букв, а базисом группы $G_{1}$ по этой системе является группа $G_{2}$, заданная образующими $a_{1}, a_{2}, \ldots, a_{n}, l, r, c$ и определяющими соотношениями (i) и (ii).

Ниже мы докажем, что в групе $G_{2}$ буква $c$ является правильной проходной буквой, а базисом при этом будет группа $G_{3}$, заданная образующими $a_{1}, a_{2}, \ldots, a_{n}, l, r$ и определяюшими соотношениями (i). Наконец, базисом групшы $G_{3}$ по системе проходных букв $a_{1}, \ldots, a_{n}$ является свободная группа $G_{0}$ с образующими $l$ и $r$. Так как слова $l$ и $r^{m+1}$ (так же как и слова $l^{m+1}$ и $r$ ) являются свободными образующими порождаемой ими подгруппы в свободной группе, то буквы $a_{1}, \ldots, a_{n}$ образуют в группе $G_{3}$ правильную систему проходных букв.

Слово $W\left(a_{1}, \ldots, a_{n}, l E_{1} r, l^{2} E_{2} r^{2}, \ldots, l^{m} E_{m} r^{m}\right)$ назовем несократимым нацело, если в нем нет двух стоящих рядом взаимно обратных компонент. Мы докажем, что для любых непустых слов $E_{1}, \ldots, E_{m}$ непустое несократимое нацело слово вида $W\left(a_{1}, \ldots, a_{n}, l E_{1} r, l^{2} E_{2} r^{2}, \ldots, l^{m} E_{m} r^{m}\right)$ не может быть равно 1 в групше $G_{3}$.

Допустим, что непустое несократимое нацело слово

$$
W\left(a_{1}, \ldots, a_{n}, l E_{1} r, l^{2} E_{2} r^{2}, \ldots, l^{m} E_{m} r^{m}\right)
$$


равно 1 в группе $G_{3}$. Тогда в слово $W$ входит некоторая буква $a_{i}$. Так как буквы $a_{1}, \ldots, a_{n}$ образуют в группе $G_{3}$ правильную систему проходных букв с базой $G_{0}$, то по лемме Бриттона слово $W$ имеет вид $W=W_{1} a_{i}^{\delta} T a_{i}^{-\delta} W_{2}$, где буквы $a_{j}$ не входят в $T$ и $T=\varphi\left(l, r^{m+1}\right)$ при $\delta=1$ и $T=\varphi\left(l^{m+1}, r\right)$ при $\delta=-1$. Очевидно, буквы $a_{i}^{\delta}$ и $a_{i}^{-\delta}$ берутся из соседних вхождений компонент $a_{1}, \ldots, a_{n}, l^{1} E_{1} r^{1}, \ldots, l^{m} E_{m} r^{m}$. Невозможность этого вытекает из следующего утверждения, которое нам понадобится и позже.

Лемма 6.2. Пусть $E_{1}, \ldots, E_{m}$ - непустые слова в алфавите полугруппь П, $W=\varphi\left(a_{1}, \ldots, a_{n}, l E_{1} r, l^{2} E_{2} r^{2}, \ldots, l^{m} E_{m} r^{m}\right)$ - несократимое начело слово. Если при некоторых $P$ и $Q$ выполняется равенство

$$
P W Q=X_{1} a_{i}^{\delta} T a_{i}^{-\delta} X_{2},
$$

где $\delta= \pm 1$ u $T=\psi\left(l, r^{m+1}\right)$ nрu $\delta=1 u T=\psi\left(l^{m+1}, r\right)$ nри $\delta=-1$, mо оба выделенные вхождения букв $a_{i}^{\delta} и a_{i}^{-\delta}$ не могут содержсться одновременно в отрезке $W$.

ДокАЗАТЕЛЬСтво. Предположим противное, т.е. обе эти буквы входят в $W$. Достаточно рассмотреть случай $\delta=1$. Тогда слово $W$ имеет вид $W=W_{1} a_{i} T a_{i}^{-1} W_{2}$. В силу несократимости нацело слова $W$ хотя бы одна из этих компонент имеет вид $l^{j} E_{j} r^{j}$. Здесь надо рассмотреть следующие ниже 3 случая.

1. $a_{i}$ есть целая компонента, а $a_{i}^{-1}$ является первой $a$-буквой соседней справа компоненты вида $\left(l^{j} E_{j} r^{j}\right)^{-1}$. В таком случае имеем $T=r^{-j}$, где $1 \leqslant j \leqslant m$ и $r^{-j}$ равно слову $\varphi\left(l, r^{m+1}\right)$ в свободной группе $G_{0}$ с порождающими $l$ и $r$, что невозможно.

2. $a_{i}^{-1}$ есть целая компонента, а $a_{i}$ является последней $a$-буквой соседней слева компоненты вида $l^{j} E_{j} r^{j}$. В таком случае имеем $T=r^{j}$ и слово $r^{j}$ равно слову $\varphi\left(l, r^{m+1}\right)$ в свободной группе $G_{0}$, что невозможно.

3. $a_{i}$ является последней $a$-буквой некоторой компоненты вида $l^{j} E_{j} r^{j}$, а $a_{i}^{-1}$ является первой $a$-буквой соседней справа компоненты вида $\left(l^{s} E_{s} r^{s}\right)^{-1}$. Тогда слово $T$ имеет вид $r^{j-s}$, где $0<|j-s|<m$, и равно $\varphi\left(l, r^{m+1}\right)$ в групе $G_{0}$, что также невозможно.

Полученное нами противоречие завершает доказательство леммы 6.2.

В силу леммы Бриттона непустое несократимое нацело слово вида $W\left(a_{1}, \ldots, a_{n}\right.$, $\left.l E_{1} r, l^{2} E_{2} r^{2}, \ldots, l^{m} E_{m} r^{m}\right)$ не может быть равно 1 в группе $G_{3}$. Отсюда следует, что слова $a_{1}, \ldots, a_{n}, l E_{1} r, l^{2} E_{2} r^{2}, \ldots, l^{m} E_{m} r^{m}$, так же как и слова $a_{1}, \ldots, a_{n}, l F_{1} r, l^{2} F_{2} r^{2}$ $\ldots, l^{m} F_{m} r^{m}$, являются свободными образующими порождаемой ими подгруппы в группе $G_{3}$. Следовательно, буква $c$ есть правильная проходная буква в группе $G_{2}$.

Через $L$ и $R$ (с индексами или без них) будем обозначать слова вида $\varphi(l, c)$ и $\varphi(r, c)$, соответственно.

Лемма 6.3. Если $X$ и $Y$ - слова в алфавите полугруппь П и $X=Y$ в П, то можно указать такие слова $L$ и $R$, что $X=L Y R$ в группе $G$.

ДокАЗАТЕльство. Если переход $X \rightarrow Y$ является элементарным преобразованием в полугруппе П, то для некоторых слов $U, V$ и некоторого числа $i$ вьполнены равенства $X=U E_{i} V$ и $Y=U F_{i} V$, или наоборот. Тогда в группе $G$ получаем равенства

$$
X=U E_{i} V=U\left(l^{-i} c l^{i}\right) F_{i}\left(r^{i} c^{-1} r^{-i}\right) V=L_{1} U F_{i} V R_{1}=L_{1} Y R_{1} .
$$


Доказательство леммы 6.3 завершается индукцией по числу элементарных преобразований, необходимых для преобразования слова $X$ в слово $Y$ в полугруппе П.

ЛЕмма 6.4. Пусть $X-$ слово в алфавите полугруппь П. Если $X=q$ в П, то $\left(X^{-1} t X\right) k\left(X^{-1} t^{-1} X\right)=k$ в грynne $G$.

ДокАЗАТЕЛЬСТво. Пусть $X=q$ в полугруппе П. Тогда по лемме 6.3 найдутся такие слова $L$ и $R$, что $X=L q R$ в группе $G$. Используя это равенство, получаем в группе $G$

$$
\begin{aligned}
\left(X^{-1} t X\right) k\left(X t^{-1} X\right) & =\left(R^{-1} q^{-1} L^{-1} t L q R\right) k\left(R^{-1} q^{-1} L^{-1} t^{-1} L q R\right) \\
& =R^{-1} q^{-1} t q k q^{-1} t^{-1} q R=R^{-1} k R=k .
\end{aligned}
$$

Лемма 6.5. Если $X$ - непустое несократимое слово в алфавите группь $G$, не содержсащее букв $c, r, l, t, k, u\left(X^{-1} t X\right) k=k\left(X^{-1} t X\right)$ в группе $G$, то $X$ - слово в положительном алфавите $u X=q$ в полугруппе $\Pi$.

ДокАЗАтЕльство. Пусть $k^{-1}\left(X^{-1} t X\right) k\left(X^{-1} t^{-1} X\right)=1$ в группе $G$. Тогда по лемме Бриттона слово $X^{-1} t X$ равно в группе $G_{1}$ некоторому слову $\varphi\left(c, r, q^{-1} t q\right)$. Можно считать, что число множителей в слове $\varphi\left(c, r, q^{-1} t q\right)$ минимально возможное. Обозначим $D \rightleftharpoons \varphi^{-1}\left(c, r, q^{-1} t q\right)$. По лемме Бриттона из равенства $X^{-1} t X D=1$ в группе $G_{1}$ следует, что $X^{-1} t X D=X_{1} t^{\delta} T t^{-\delta} Z$, где $T=\psi(c, l)$ в $G_{2}$ при некотором $\psi$.

Допустим, что обе буквы $t^{\delta}$ и $t^{-\delta}$ входят в $D$. Тогда $T=q R q^{-1}$ при некотором $R$. Используя также равенство $T=\psi(c, l)$ в $G_{2}$, получаем

$$
\begin{aligned}
D & =D_{1}\left(q^{-1} t q\right)^{\delta} R\left(q^{-1} t q\right)^{-\delta} D_{2}=D_{1} q^{-1} t^{\delta} T t^{-\delta} q D_{2} \\
& =D_{1} q^{-1} t^{\delta} \psi(c, l) t^{-\delta} q D_{2}=D_{1} q^{-1} \psi(c, l) q D_{2}=D_{1} q^{-1} T q D_{2}=D_{1} R D_{2},
\end{aligned}
$$

а это противоречит предположению о минимальности числа сомножителей в слове $\varphi\left(c, r, q^{-1} t q\right)$.

Значит, буква $t^{\delta}$ есть первая буква $t$ в слове $X^{-1} t X D$, т.е. $\delta=1, D=R^{-1} q^{-1} t^{-1} q D_{1}$ и $T=X R^{-1} q^{-1}$ при некотором $R=\varphi(c, r)$. Тогда по лемме Бриттона имеем в группе $G_{2}$ равенство $X R^{-1} q^{-1}=\psi(c, l)$ при некотором $\psi(c, l)$. Это означает, что при некоторых $R$ и $L$ в группе $G_{2}$ имеет место равенство $X^{-1} L q R=1$.

Индукцией по числу $\alpha$ вхождений букв $c^{ \pm 1}$ в слово $L R$ докажем, что для произвольного непустого несократимого слова $X$ в алфавите $\left\{a_{j}, a_{j}^{-1}\right\}$ и произвольного непустого слова $Q$ в алфавите полугруппь П равенство

$$
X^{-1} L Q R=1
$$

в группе $G$ влечет, что $X$ не содержит отрицательных степеней и в полугруппе П выполнено равенство $X=Q$.

Будем считать, что слова $L$ и $R$ несократимы.

Если число $\alpha$ равно нулю, то $L=l^{\beta}$ и $R=r^{\gamma}$. Тогда из равенства $X^{-1} L Q R=1$ в группе $G_{2}$ следует, что $X^{-1} Q$ равно 1 в свободной группе, т.е. $X \mp Q$, так как $X$ и $Q$ - несократимые слова. 
Пусть буква $c$ входит в слово $X^{-1} L Q R$. Тогда по лемме Бриттона имеем $X^{-1} L Q R$ = $Y_{1} c^{\delta} T c^{-\delta} Y_{2}$, где в группе $G_{3}$ вьполнено равенство

$$
T \varphi\left(a_{1}, \ldots, a_{n}, l E_{1} r, l^{2} E_{2} r^{2}, \ldots, l^{m} E_{m} r^{m}\right)=1
$$

при $\delta=-1$ или равенство

$$
T \varphi\left(a_{1}, \ldots, a_{n}, l F_{1} r, l^{2} F_{2} r^{2}, \ldots, l^{m} F_{m} r^{m}\right)=1
$$

при $\delta=1$. Эти два случая аналогичны.

Рассмотрим случай $\delta=-1$. Будем считать, что число сомножителей в слове $\varphi\left(a_{1}, \ldots, a_{n}, l E_{1} r, l^{2} E_{2} r^{2}, \ldots, l^{m} E_{m} r^{m}\right)$ минимально. Пусть

$$
\varphi\left(a_{1}, \ldots, a_{n}, l E_{1} r, l^{2} E_{2} r^{2}, \ldots, l^{m} E_{m} r^{m}\right)=W_{1} D,
$$

где $W_{1}$ есть максимальное начало, состоящее из $a$-букв. Тогда равенство (24) группы $G_{3}$ запишется в виде

$$
T W_{1} D=1
$$

Допустим, что слово $T$ содержится в $L$. Тогда в $L$ содержится и слово $c^{-1} T c, T=l^{\beta}$ и по лемме 6.2 из равенства

$$
l^{-\beta} \varphi\left(a_{1}, \ldots, a_{n}, l E_{1} r, l^{2} E_{2} r^{2}, \ldots, l^{m} E_{m} r^{m}\right)=1
$$

в группе $G_{3}$ следует, что слово $\varphi\left(a_{1}, \ldots, a_{n}, l E_{1} r, l^{2} E_{2} r^{2}, \ldots, l^{m} E_{m} r^{m}\right)$ пусто, т.е. $l^{-\beta}=1$ в группе $G_{3}$. Значит, $\beta=0$ и слово $L$ сократимо.

Аналогично приводится к противоречию предположение, что слово $T$ содержится в $R$. Остается рассмотреть случай, когда подслово $c^{-1} T c$ начинается в $L$ и заканчивается в $R$. Тогда имеем

$$
T=l^{\beta} Q r^{\gamma}, \quad L=L_{1} c^{-1} l^{\beta}, \quad R=r^{\gamma} c R_{1}
$$

и соотношение $(25)$ группы $G_{3}$ запишется в виде

$$
l^{\beta} Q r^{\gamma} W_{1} D=1
$$

где слово $D$ или пусто, или начинается с некоторого множителя $\left(l^{i} E_{i} r^{i}\right)^{\delta}$. По лемме Бриттона имеем

$$
l^{\beta} Q r^{\gamma} W_{1} D=Z_{1} a_{j}^{\delta} T a_{j}^{-\delta} Z_{2},
$$

где $\delta= \pm 1$ и в группе $G_{0}$ вьполнено равенство $T=\psi\left(l, r^{m+1}\right)$ при $\delta=1$ или равенство $T=\psi\left(l^{m+1}, r\right)$ при $\delta=-1$. Так как число множителей в слове $W_{1} D$ минимально, то в силу леммы $6.2 W_{1} D$ не может содержать обе буквы $a_{j}^{\delta}$ и $a_{j}^{-\delta}$. Так как слово $Q$ также не может содержать одновременно обе эти буквы, то $a_{j}^{\delta}$ есть последняя буква в слове $Q$, т.е. $\delta=1$.

Если слово $W_{1}$ непусто, то имеем $Q=Q^{\prime} a_{j}, W_{1}=a_{j}^{-1} W_{1}^{\prime}$ и $T=r^{\gamma}$, причем, $T \equiv \varphi\left(l, r^{m+1}\right)$. Отсюда следует, что $\gamma=\gamma^{\prime}(m+1)$ и $l^{\beta} Q r^{\gamma} W_{1} D=l^{\beta} Q^{\prime} r^{\gamma^{\prime}} W_{1}^{\prime} D$ в $G_{3}$. 
Если $W_{1}^{\prime}$ также непусто, то мы опять можем повторить наши рассуждения. Проведя это рассуждение столько раз, какова длина слова $W_{1}$, получим в результате

$$
\gamma=\gamma_{1}(m+1)^{\left|W_{1}\right|}
$$

$Q=Q_{1} W_{1}^{-1}$ и $l^{\beta} Q r^{\gamma} W_{1} D=l^{\beta} Q_{1} r^{\gamma_{1}} D$ в групше $G_{3}$.

Если при этом слово $D$ пусто, то $Q_{1}$ - пустое слово и $l^{\beta} r^{\gamma_{1}}=1$ в группе $G_{3}$. Но тогда $\beta=\gamma_{1}=0$, а значит, и $\gamma=0$. В таком случае в силу (26) вместо равенства (23) в группе $G_{2}$ получаем равенство

$$
1=X^{-1} L_{1} c^{-1} Q c R_{1}=X^{-1} L_{1} Q R_{1},
$$

где слово $L_{1} R_{1}$ имеет менњше вхождений букв $c^{ \pm 1}$, чем слово $L R$. По индуктивному предположению получаем, что $X$ есть слово в положительном алфавите и в полугруппе П вьполнено равенство $X=Q$.

Пусть слово $D$ непусто. Тогда $D=\left(l^{i} E_{i} r^{i}\right)^{\delta} W_{2} D_{1}$, где $W_{2}$ есть максимальньй отрезок, состоящий из компонент $a_{j}^{ \pm 1}$. Так как $Q_{1}$ - слово в положительном алфавите и слово $E_{i}$ непусто, то, используя лемму 6.2 , получаем:

$$
\delta=-1, Q_{1}=Q_{1}^{\prime} a_{j}, E_{i}=E_{i}^{\prime} a_{j} \text { и } r^{\gamma_{1}-i}=\varphi\left(l, r^{m+1}\right) \text { в групше } G_{0} .
$$

Тогда $\gamma_{1}-i$ должно делиться на $(m+1)$, и далее аналогично предыдушему получим, что $Q_{1}=Q_{2} E_{i}, \gamma_{1}-i=(m+1)^{\left|E_{i}\right|} \gamma_{2}$ и $l^{\beta} Q r^{\gamma} W_{1} D=l^{\beta} Q_{2} r^{\gamma_{2}} l^{-i} W_{2} D_{1}$ в группе $G_{3}$, где по условию $W_{2}$ есть максимальный отрезок, состоящий из компонент $a_{j}^{ \pm 1}$. Если $W_{2}$ непусто, то, повторив приведенные выше рассуждения по отношению к равенству $l^{\beta} Q_{2} r^{\gamma_{2}} l^{-i} W_{2} D_{1}=1$, получим $Q_{2} \mp Q_{3} W_{2}^{-1}$ и при этом вьведем в группе $G_{3}$ равенство

$$
l^{\beta} Q r^{\gamma} W_{1} D=l^{\beta} Q_{3} r^{\gamma_{3}} l^{-i(m+1)^{\left|W_{2}\right|}} D_{1}=1,
$$

где $\gamma_{2}=\gamma_{3}(m+1)^{\left|W_{2}\right|}$.

Сначала докажем, что слово $D_{1}$ пусто. Допустим, что оно непусто. Тогда $D_{1}$ имеет вид $\left(l^{i_{1}} E_{i_{1}} r^{i_{1}}\right)^{\eta} Z$, где $\eta= \pm 1$.

В силу равенства (28) и леммы Бриттона в слово $l^{\beta} Q_{3} r^{\gamma_{3}} l^{-i(m+1)^{\left|W_{2}\right|}} D_{1}$ должно входить подслово вида $a_{j}^{\delta} T a_{j}^{-\delta}$, где $\delta= \pm 1$ и в свободной групше $G_{0}$ выполнено равенство $T=\psi\left(l, r^{m+1}\right)$ при $\delta=1$ или равенство $T=\psi\left(l^{m+1}, r\right)$ при $\delta=-1$. По лемме 6.2 подслово такого вида не может содержаться в $D_{1}$. Поэтому последняя буква слова $Q_{3}$ есть $a_{j}^{\delta}$. Значит, $\delta=1, \eta=-1$ и слово

$$
T=r^{\gamma_{3}} l^{-i(m+1)^{\left|W_{2}\right|}} r^{-i_{1}}
$$

равно в свободной группе $G_{0}$ некоторому слову $\psi\left(l, r^{m+1}\right)$. Но это невозможно, так как $0<i_{1}<m+1$.

Итак, $D_{1}$ пусто. Тогда пусто и слово $Q_{3}$, т.е. $Q_{2}=W_{2}^{-1}$. Кроме того, имеем равенства $\gamma_{3}=0$ и $\beta=i(m+1)^{\left|W_{2}\right|}$. Равенство $\gamma_{3}=0$ влечет $\gamma_{2}=0$. Следовательно, $i=\gamma_{1}$, откуда в силу $(27)$ получаем

$$
\gamma=i(m+1)^{\left|W_{1}\right|} .
$$


Вернемся к слову $X^{-1} L Q R$. Имеем $Q=Q_{1} W_{1}^{-1}=Q_{2} E_{i} W_{1}^{-1}=W_{2}^{-1} E_{i} W_{1}^{-1}$ и следующие равенства в группе $G_{2}$ :

$$
\begin{aligned}
X^{-1} L Q R & =X^{-1} L_{1} c^{-1} l^{\beta} W_{2}^{-1} E_{i} W_{1}^{-1} r^{\gamma} c R_{1} \\
& =X^{-1} L_{1} c^{-1} W_{2}^{-1} l^{i} E_{i} r^{i} W_{1}^{-1} c R_{1}=X^{-1} L_{1} W_{2}^{-1} c^{-1} l^{i} E_{i} r^{i} c W_{1}^{-1} R_{1} \\
& =X^{-1} L_{1} W_{2}^{-1} l^{i} F_{i} r^{i} W_{1}^{-1} R_{1}=X^{-1} L_{1} l^{\beta} W_{2}^{-1} F_{i} W_{1}^{-1} r^{\gamma} R_{1} .
\end{aligned}
$$

Положим

$$
Q^{\prime} \rightleftharpoons W_{2}^{-1} F_{i} W_{1}^{-1}, \quad L^{\prime} \rightleftharpoons L_{1} l^{\beta}, \quad R^{\prime} \rightleftharpoons r^{\gamma} R_{1} .
$$

И силу (23) имеем $X^{-1} L^{\prime} Q^{\prime} R^{\prime}=1$ в группе $G_{2}$, откуда по индуктивному предположению следует равенство $Q^{\prime}=X$ в полугруппе П. Так как $Q=Q^{\prime}$ в полугруппе П, то $X=Q$ в полугруппе П.

Тем самьм доказано, что для произвольного непустого несократимого слова $X$ в алфавите группь $G$, не содержащего букв $c, r, l, t, k$, и произвольного непустого слова $Q$ в алфавите полугруппь П равенство $X^{-1} L Q R=1$ в группе $G$ влечет равенство $X=Q$ в полугруппе $\Pi$.

Поэтому из доказанного выше равенства $X^{-1} L q R=1$ в группе $G_{2}$ следует равенство $X=q$ в полугруппе П. Это завершает доказательство леммы 6.5 .

Из лемм 6.4 и 6.5 сразу получаем следующую теорему.

Теорема 6.6. Пусть $X-$ слово в алфавите полугруппь П. Тогда имеет место әквивалентность:

$$
X=q \text { в } \Pi \Longleftrightarrow\left(X^{-1} t X\right) k\left(X^{-1} t^{-1} X\right) k^{-1}=1 \text { в груnпе } G .
$$

Так как по условию конечно определенная полугруппа П имеет неразрешимую проблему равенства $X=q$ фиксированному слову $q$, то получаем, что в группе $G$ неразрешима проблема распознавания равенства уже для слов вида $X^{-1} t X k X^{-1} t^{-1} X k^{-1}$, где $X$ - произвольное непустое слово в алфавите полугруппы П. Это завершает доказательство теоремы 6.5.

В дальнейшем нам понадобится следуюшее свойство слов указанного вида.

Лемма 6.6. Пусть $X-$ слово в алфавите полугруппьц П $u X \neq q$ в П. Тогда слово $X^{-1} t X k X^{-1} t^{-1} X k^{-1}$ имеет в группе $G$ бесконечный порядок.

ДокАЗАТЕЛЬСТвО. Допустим, что для некоторого натурального числа $n>0$ выполняется равенство $\left(X^{-1} t X k X^{-1} t^{-1} X k^{-1}\right)^{n}=1$. Тогда по лемме Бриттона найдется такое слово $\varphi\left(c, r, q^{-1} t q\right)$, что $X^{-1} t X=\varphi\left(c, r, q^{-1} t q\right)$ в группе $G_{1}$, т.е. $X^{-1} t X$ коммутирует с $k$. Тогда по лемме 6.5 получим, что $X=q$ в П. Это завершает доказательство леммы 6.6 .

Если в качестве полугрупшы П взять указанную в теореме 2.3 полугруппу с 2 образуюшими и 3 определяюшими соотношениями, имеюшую неразрешимую проблему равенства некоторому фиксированному слову $q$, то приведенная нами конструкция дает группу $G$ с 14 определяюшими соотношениями, имеюшую неразрешимую проблему равенства слов. 
В работе [25] было показано, что можно так закодировать буквы $a_{1}, a_{2}, t, k$ в двубуквенном алфавите $\{a, b\}$, что заменив эти буквы их кодами в определяющих соотношениях группы, можно четыре коммутации этих букв с буквой $c$ заменить двумя $a c=c a, b c=c b$. При этом новая группа будет содержать группу $G$ в качестве подгруппы и, следовательно, в ней будет неразрешима проблема равенства слов. Полученная группа будет задаваться 12 определяюшими соотношениями. Разрешимость проблемы равенства для групп с одним определяющим соотношением была установлена В. Магнусом еще в 1932 году в работе [50]. Доказательство этого результата изложено ниже в разделе 7. Вопрос о сушествовании групп с неразрештмой проблемой равенства слов, заданных $k$ определяюшими соотношениями, при $2 \leqslant k \leqslant 11$ пока остается открытьм.

Из неразрешимости проблемы равенства для данной групшы непосредственно следует неразрешимость для нее и проблемы сопряэенности, которая состоит в отыскании алгоритма для распознавания по произвольной паре слов, сопряжены они в этой группе или нет. В работе [71] П. С. Новиков построил довольно простой пример конечно определенной группы с неразрешимой проблемой сопряженности, причем, как было показано в работе [33], в этой группе проблема равенства слов разрешима.

Следует также отметить результат Е. Скотт [95], построившей пример конечно определенной простой группы с неразрешимой проблемой сопряженности. Этот результат интересен в связи с отмеченным ниже алгоритмом, решающим проблему равенства слов для простых групп.

Результат П. С. Новикова о неразрешимости проблемы равенства слов теории групा и другие результаты, полученные затем в этом направлении (о них будет сказано ниже) позволили математикам уже в 50-х годах осознать, что неразрешимые алгоритмические проблемы достаточно шшироко распространены в математике и встречаются среди весьма актуальных алгоритмических проблем, относящихся к фундаментальным понятиям математики.

В середине 50-х годов естественно возник вопрос о том, может ли конечно определенная группа с неразрешимой проблемой равенства слов удовлетворять нетривиальному тождеству. Дело в том, что как примеры П. С. Новикова, так и примеры, построенные вскоре после него другими авторами, содержали системы проходных букв и поэтому не могли удовлетворять никакому нетривиальному тождеству.

Среди групп, удовлетворяющих нетривиальному тождеству, особое место занимают периодические групшы. Проблема равенства в этих группах тесно связана с известной проблемой Бернсайда о том, являются ли конечными все конечно порожденные группы, удовлетворяющие тождеству вида $x^{n}=1$. Бесконечность этих групп при достаточно больших периодах $n$ удалось доказать только после того, как были найдены некоторые критерии неравенства единице для слов в этих группах. Такие условия впервые были получены для нечетных $n \geqslant 4381$ в 1968 году при доказательстве бесконечности этих групп в совместной работе П. С. Новикова и С. И. Адяна [74]. Совершенно естественно, что попутно для этих групп было получено положительное решение как проблемы равенства [75], так и проблемы сопряженности [76]. Более того, каждый раз, когда расширяется гранища применения изложенного в [74] метода Новикова-Адяна, для нового более широкого класса групп попутно получается решение алгоритмических проблем равенства и сопряженности. Это было так в моногра- 
фии С.И. Адяна [13], где метод был распространен на нечетные периоды $n \geqslant 665$, в работах А. Ю. Ольшанского и его учеников [78], где метод Новикова-Адяна был переведен на язык диаграмм ван Кампена, а также в работе И. Г. Лысенка [48], где он был распространен на четные периоды вида $2^{4} n \geqslant 8000$.

Первые примеры конечно порожденных групп, имеюших неразрешимую проблему равенства и удовлетворяющих нетривиальным тождествам, были найдены в 1969 году [11], [12] с использованием указанного метода Новикова-Адяна. Эти группы определяются рекурсивным множеством тождественных соотношений в алфавите от двух переменных $x, y$ и без дополнительных определяюших соотношений. На поставленный в этих работах вопрос о существовании конечно порож денной группы, заданной конечным числом тождеств (а значит, и одним тождеством) и имеющей неразрешимую проблему равенства слов, положительньй ответ был получен Ю. Г. Клейманом в работе [44]. Там же был построен пример группы, заданной тремя образуюшими элементами и рекурсивньм множеством определяюших соотношений, которая имеет разрешимую проблему равенства слов, в то время как невозможен алгоритм, который бы распознавал по данному тождественному соотношению, выполнено оно в этой группе или нет.

Примеры 2-порожденных групп с неразрешимой проблемой равенства, определенных тождеством вида $x^{n}=1$ и рекурсивным множеством определяюших соотношений, были получены С.И. Адяном в работе [16]. Позже М.В. Сапир [90] построил конечно определенную групу с неразрешимой проблемой равенства слов в многообразии, заданном двумя тождествами $x^{n p}=1$ и $\left[x^{n}, y^{n}\right]=1$ при нечетных $n \geqslant 665$ и любых $p>1$, взаимно простых с $n$.

В. Н. Ремесленников в 1973 году [86] построил пример конечно определенной группы в многообразии разрешимых групп ступени 5 с неразрешимой проблемой равенства слов. В этом примере к конечному числу определяющих соотношений добавляется соотношение разрешимости ступени 5. Аналогичньй результат для разрешимых групш ступени 3 был получен В. И. Епанчинцевым и Г. П. Кукиным [32]. Наконец, О. Г. Харлампович [43] впервые построила пример конечно определенной группы с неразрешимой проблемой равенства, в которой выполняется тождественное соотношение разрешимости ступени 3

$$
\left[[[x, y],[u, v]],\left[\left[x_{1}, y_{1}\right],\left[u_{1}, v_{1}\right]\right]\right]=1 \text {. }
$$

Как было отмечено в разделе $3, \Phi$. Холл [38] ранее доказал, что проблема равенства для конечно порожденных разрешимых групп ступени 2 решается положительно, так как они финитно аппроксимируемы.

\section{7. Группы с одним определяющим соотношением}

Пусть группы $G_{1}$ и $G_{2}$ заданы образующими и определяющими соотношениями:

$$
G_{1}=\left\langle\left\langle\mathcal{S}_{1} \mid \mathscr{D}_{1}\right\rangle\right\rangle, \quad G_{2}=\left\langle\left\langle\mathcal{S}_{2} \mid \mathscr{D}_{2}\right\rangle\right\rangle .
$$

Если множества $\mathcal{S}_{1}$ и $\mathcal{S}_{2}$ не пересекаются, то группа

$$
G_{1} * G_{2} \rightleftharpoons\left\langle\left\langle\mathcal{S}_{1} \cup \mathcal{S}_{2} \mid \mathscr{D}_{1} \cup \mathscr{D}_{2}\right\rangle\right\rangle
$$

называется свободньм произведением групп $G_{1} u G_{2}$. 
Очевидно, любое слово $X$ равно в группе $G_{1} * G_{2}$ некоторому слову $W$, которое находится в приведенной форме, т.е. имеет вид

$$
W=W_{1} \ldots W_{n-1} W_{n} \quad(n \geqslant 1),
$$

где каждое $W_{j}$ есть слово в алфавите одной из групп $G_{1}$ или $G_{2}$, причем $W_{j}$ и $W_{j+1}$ - слова в разных алфавитах и при $n>1$ все $W_{j}$ не равны единице в соответствующей группе.

Следуюшая теорема о приведенной форме для свободных произведений доказывается элементарно.

TeOpema 7.1. Ecлu

$$
W=W_{1} \ldots W_{n-1} W_{n}
$$

$$
U=U_{1} \ldots U_{m-1} U_{m}
$$

- приведенные формы слов $W$ и в свободном произведении $G_{1} * G_{2}$, то $W$ равно $U$ в $G_{1} * G_{2}$ тогда и только тогда, когда $n=m$ и при любом $j=1, \ldots, n$ слово $W_{j}$ равно $U_{j}$ в соответствующей группе.

Пусть в конечно определенных группах

$$
G_{1}=\left\langle\left\langle\mathcal{S}_{1} \mid \mathscr{D}_{1}\right\rangle\right\rangle \text { и } G_{2}=\left\langle\left\langle\mathcal{S}_{2} \mid \mathscr{D}_{2}\right\rangle\right\rangle
$$

выделены две подгруппы

$$
H_{1}=\left\langle A_{1}, \ldots, A_{k}\right\rangle_{G_{1}} \text { и } H_{2}=\left\langle B_{1}, \ldots, B_{k}\right\rangle_{G_{2}},
$$

причем соответствие $A_{i} \stackrel{\varphi}{\mapsto} B_{i}(i=1, \ldots, k)$ порождает изоморфное отображение $G_{1}$ на $G_{2}$.

ОПРЕДЕЛЕНИЕ. Свободньм произведением групп $G_{1}$ и $G_{2}$ с объединенными подгруппами $H_{1} \cong H_{2}$ назьвается группа

$$
\left\langle\left\langle\mathcal{S}_{1} \cup \mathcal{S}_{2} \mid \mathscr{D}_{1} \cup \mathscr{D}_{2}, A_{1}=B_{1}, \ldots, A_{k}=B_{k}\right\rangle\right\rangle .
$$

Можно считать, что порождающие выделенных подгрупп $A_{1}, \ldots, A_{k}$ и $B_{1}, \ldots, B_{k}$ входят в число исходных образуюших соответствуюших групп. Для этого достаточно добавить в задания групп $G_{1}$ и $G_{2}$ новые образующие $c_{1}, \ldots, c_{k}$ и приравнять их к соответствуюшим словам $A_{1}, \ldots, A_{k}$ и $B_{1}, \ldots, B_{k}$. Поэтому мы будем считать, что заданы две конечно определенные групшы

$$
G_{1}=\left\langle\left\langle\mathcal{S}_{1}^{\prime} \cup \mathcal{S}_{0} \mid \mathscr{D}_{1}\right\rangle\right\rangle \text { и } G_{2}=\left\langle\left\langle\mathcal{S}_{2}^{\prime} \cup \mathcal{S}_{0} \mid \mathscr{D}_{2}\right\rangle\right\rangle,
$$

причем $\mathcal{S}_{i}=\mathcal{S}_{i}^{\prime} \cup \mathcal{S}_{0}, \mathcal{S}_{1}^{\prime} \cap \mathcal{S}_{2}^{\prime}=\varnothing$ и объединяемые подгруппы $H_{1}$ и $H_{2}$ порождаются образуюшими $\mathcal{S}_{0}=\left\{c_{1}, \ldots, c_{k}\right\}$ в группах $G_{1}$ и $G_{2}$, соответственно. Изоморфизм подгрупп $H_{1}$ и $H_{2}$ означает, что для любого слова $X$ в алфавите $\mathcal{S}_{0}$

$$
X=1 \text { в } G_{1} \Longleftrightarrow X=1 \text { в } G_{2} \text {. }
$$

Тогда свободное произведение групп $G_{1}$ и $G_{2}$ с объединенными подгруппами, порожденными в них множеством $\mathcal{S}_{0}$, имеет задание

$$
\left\langle\left\langle\mathcal{S}_{1}^{\prime} \cup \mathcal{S}_{0} \cup \mathcal{S}_{2}^{\prime} \mid \mathscr{D}_{1} \cup \mathscr{D}_{2}\right\rangle\right\rangle .
$$


Теорема 7.2. Если $G_{3}$ есть свободное произведение (3) групп $G_{1}$ и $G_{2}$ с обтединенными подгруппами, то кажсдая из этих групп естественным образом вложена в $G_{3}$.

ДокАЗАТЕльСтво. Алфавиты $\mathcal{S}_{i}$ упорядочим так, чтобы все буквы из $\mathcal{S}_{0}$ предшествовали остальным буквам. Будем рассматривать лексикографическое упорядочение множества $\mathcal{S}_{i}^{*}$ слов в алфавите $\mathcal{S}_{i}$, удовлетворяющее дополнительному условию, что все слова в алфавите $\mathcal{S}_{0}$ предшествуют всем словам, содержашим буквы из алфиавита $\mathcal{S}_{i}^{\prime}$. Через $\stackrel{i}{=}$ будем обозначать равенство слов в групше $G_{i}(i=1,2)$. Для любого слова $X \in S_{i}^{*}$ выберем в смежном классе $X H_{i}$ наименьшее в указанном выше лексикографическомпорядке слово $Y$ в алфавите $\mathcal{S}_{i}$ и положим $\psi(X) \rightleftharpoons Y$. Очевидно, для любого слова $X$ в алфавите $\mathcal{S}_{i}$ можно указать представление

$$
X \stackrel{i}{=} \psi(X) A, \quad \text { где } A \in \mathcal{S}_{0}^{*} .
$$

Для любых двух равных в $G_{i}$ слов $U$ и $V$ имеем $\psi(U)=\psi(V)$.

Если существует слово из $\mathcal{S}_{0}^{*}$, равное данному слову $X$ в группе $G_{i}$, то $X \in H_{i}$ и, следовательно, $\psi(X)=1$.

Для слов $X \in H_{i}$ введем также функцию $\bar{X}$, которую определим как наименьшее в указанном выше лексикографическом порядке слово в алфавите $\mathcal{S}_{0}$, равное слову $X$ в группе $G_{i}$. Если такого слова в алфавите $\mathcal{S}_{0}$ нет, то считаем, что функция $\bar{X}$ не определена. Очевидно, в равенстве (4) всегда можно считать, что $\bar{A}=A$.

Теперь для произвольного слова $X$ в образующих группы $G_{3}$ определим стандартное представление $\varphi(X)$, равное $X$ в группе $G_{3}$ и имеюшее вид

$$
\varphi(X)=C_{1} C_{2} \ldots C_{r} A,
$$

где $\bar{A}=A, C_{j}$ суть значения $\psi(Z)$ для некоторых слов $Z \in \mathcal{S}_{i}^{*}$ и соседние $C_{j}$ взяты из разных алфавитов $\mathcal{S}_{i}$.

Представление $\varphi(X)$ определим индукцией по числу $\gamma(X)$ множителей из различных $\mathcal{S}_{i}^{*}$, на которые можно графически разбить слово $X$.

Если $X \in \mathcal{S}_{i}^{*}$, то положим $\varphi(X) \rightleftharpoons \psi(X) A$, где $\bar{A}=A$ (см. (4)).

Если $X \neq Y E$, где $E \in \mathcal{S}_{i}^{*}$ и последняя буква слова $Y$ не принадлежит $\mathcal{S}_{i}$, то $\gamma(Y)<\gamma(X)$. Тогда по индуктивному предположению для слова $Y$ имеем равное ему в $G_{3}$ стандартное представление

$$
\varphi(Y)=C_{1} C_{2} \ldots C_{r} A,
$$

где $\bar{A}=A$, а $C_{j}$ суть значения $\psi(Z)$ для некоторых слов $Z \in \mathcal{S}_{i}^{*}$ и соседние $C_{j}$ взяты из разных алфавитов $S_{i}$.

Если $C_{r} \in \mathcal{S}_{i}^{*}$, то $C_{r} A E \in \mathcal{S}_{i}^{*}$. Тогда положим

$$
\varphi(X) \rightleftharpoons C_{1} C_{2} \ldots C_{r-1} \psi\left(C_{r} A E\right) A_{0},
$$

где

$$
C_{r} A E \stackrel{i}{=} \psi\left(C_{r} A E\right) A_{0} \& \overline{A_{0}}=A_{0} .
$$

Если же $C_{r} \notin \mathcal{S}_{i}^{*}$, то положим

$$
\varphi(X) \rightleftharpoons C_{1} C_{2} \ldots C_{r} \psi(A E) A_{0},
$$

где $A E \stackrel{i}{=} \psi(A E) A_{0}$ и $\overline{A_{0}}=A_{0}$. 
Легко проверяются следуюшие свойства представления $\varphi(X)$ :

$$
\begin{gathered}
\left(\forall E \in \mathcal{S}_{i}^{*}\right)\left(\forall D \in \mathcal{S}_{i}^{*}\right)(E \stackrel{i}{=} D \Longrightarrow \varphi(\varphi(X) E)=\varphi(\varphi(X) D)) ; \\
\left(\forall E \in \mathcal{S}_{i}^{*}\right)\left(\forall D \in \mathcal{S}_{i}^{*}\right)(\varphi(\varphi(X) E D)=\varphi(\varphi(\varphi(X) E) D)) .
\end{gathered}
$$

Индукцией по числу элементарных преобразований групшы $G_{3}$ с использованием импликаций (7) и (8) доказывается

Лемма 7.1. Если слова $X$ и $Y$ равны в группе $G_{3}, \operatorname{mo~} \varphi(X)=\varphi(Y)$.

Из этой леммы легко следует, что для любого слова $X \in \mathcal{S}_{i}^{*}$ имеет место

$$
X=1 \text { в } G_{i} \Longleftrightarrow X=1 \text { в } G_{3}
$$

т.е. группы $G_{1}$ и $G_{2}$ являются подгруппами группы $G_{3}$. Теорема 7.2 доказана.

Tеорема 7.3. Пусть группа $G_{3}$ есть свободное произведение (3) групп $G_{1}$ и $G_{2}$ с обтединенными подгруппами $H_{1}$ и $H_{2}$, порожденными в них множсеством $S_{0}$. Если в каждой группе $G_{i}(i=1,2)$ разрешимь проблема равенства слов и проблема вхождения в подгруппу $H_{i}$, то и в $G_{3}$ разрешимь проблемы равенства и вхождения в подгруппу $G_{0}$, порожденную множеством $S_{0}$.

Кроме того, если в группах $G_{1}$ и $G_{2}$ разрешимы проблемы вхождения в любие подгруппь, порожденнье подмножествами множеств образующих этих групп, то и в группе $G_{3}$ разрешима проблема вхождения в любую подгруппу, порожденную некоторыми образующими групп $G_{1} u G_{2}$.

ДокАЗАТЕЛЬСтво. Нужно доказать, что наши функции $\psi(X), \bar{X}$ и $\varphi(X)$ можно выбрать рекурсивньми. Мы выбираем последовательно представителей в правых смежных классах по подгруппе $H_{1}$ или $H_{2}$. Для эффективности этого процесса, кроме алгоритмов, решаюших проблемы равенства и вхождения в подгруппу $H_{i}$ для группы $G_{i}$, нам нужно еше уметь для данной пары слов $A, B \in S_{i}^{*}$ определять, попадают ли они в один правый смежный класс относительно $H_{i}$. Для этого надо только проверить, входит ли $A^{-1} B$ в подгруппу $H_{i}$. В силу леммы 7.1 два произвольных слова $X$ и $Y$ группы $G_{3}$ равны в $G_{3}$ в том и только том случае, если их стандартные представления совпадают графически.

Докажем второе утверждение теоремы. Пусть $G$ - подгруппа группы $G_{3}$, порожденная некоторыми образуюшими групп $G_{1}$ и $G_{2}$. Для решения проблемы вхождения в $G$ нам достаточно при построении функций $\psi(Z)$ и $\bar{Z}$ уметь проверять каждый раз возможность выбора значений этих функций из $G \cap G_{i}$ (соответственно, из $G \cap H_{i}$ ) и для произвольного слова $X$ искать стандартную форму таким образом, чтобы $\psi(Z)$ принадлежало подгруппе $G \cap G_{i}$ каждьй раз, когда есть такая возможность, т.е. если $(\exists Y)\left(Y \stackrel{i}{=} Z \& Y \in G \cap G_{i}\right)$, то $\psi(Z) \in G \cap G_{i}$. То же самое надо делать при выборе $\bar{A}$. Тогда будем иметь $X \in G$ в том и только том случае, если все компоненты стандартного представления слова $X$ принадлежат $G \cap G_{i}$. Теорема 7.3 доказана.

В начале 30-х годов В. Магнусом были доказаны две замечательные теоремы о группах с одним определяюшим соотношением - теорема о свободе и теорема о разрешимости проблемы равенства слов. 
ТЕОРемА 7.4 (теорема о свободе). Пусть

$$
G=\langle\langle t, a, b, c, \cdots \mid R(t, a, b, c, \ldots)=1\rangle\rangle,
$$

где слово $R(t, a, b, c, \ldots)$ ииклически несократимо. Любое подмножество порождающих группь $G$, не содерхащее хотя би одну букву, входящую в $R(t, a, b, c, \ldots)$, является множеством свободных порождающих в $G$.

ДокАЗАТЕЛЬСтво. Можно считать, что все буквы алфавита группы $G$ входят в слово $R$ ив $R$ входит не менее двух букв, так как в противном случае теорема очевидна. Через $\sigma_{\alpha}(W)$ будем обозначать сумму показателей буквы $\alpha$ в слове $W$. Рассмотрим два случая.

Случай 1 . Некоторая буква, входящая в $R$, например буква $t$, имеет нулевую сумму показателей. Тогда слово $R$ равно в свободной группе $F=\langle\langle t, b, c, \ldots\rangle\rangle$ произведению $\bar{R}$ множителей вида $t^{j} e^{ \pm 1} t^{-j}$, где $e$ любая из букв $a, b, c, \ldots$, отличных от $t$. Для каждой такой буквы $e$ введем новые образуюшие

$$
e_{i} \rightleftharpoons t^{i} e t^{-i}
$$

Теперь для нахождения представления $\bar{R}\left(a_{i}, b_{j}, \ldots\right)$ в новых образующих $(9)$ достаточно в слове $R$ каждое вхождение $e^{ \pm 1}$ каждой буквы $e$ заменить на $e_{i}^{ \pm 1}$, где индекс $i$ равен сумме показателей буквы $t$ в начальном отрезке слова $R$ до заменяемого вхождения $e^{ \pm 1}$. Например, для слова $R=t^{i} a^{\alpha} t^{j} b^{\beta} t^{k} a^{\gamma} t^{n}$, где $i+j+k+n=0$, будем иметь

$$
\bar{R}\left(a_{i}, b_{j}, \ldots\right)=a_{i}^{\alpha} b_{i+j}^{\beta} a_{i+j+k}^{\gamma} .
$$

Так как буква $t$ по предположению входит в слово $R(t, a, b, c, \ldots)$, то $\left|\bar{R}\left(a_{i}, b_{j}, \ldots\right)\right|<$ $|R(t, a, b, c, \ldots)|$. Заметим, что представление $\bar{E}\left(a_{i}, b_{j}, \ldots\right)$ можно указать для любого слова $E$ группы $G$ при $\sigma_{t}(E)=0$.

Допустим, что некоторое непустое и несократимое слово $H$ равно 1 в групше $G$ и не содержит одну из букв алфавита. Тогда $\sigma_{t}(H)=0$. Сначала рассмотрим подслучай, когда эта буква отлична от $t$. Без ограничения общности можно считать, что это есть буква $a$, т.е. $H=H(t, b, c, \ldots)$, и при этом буква $a$ стоит в начале слова $R$. Пусть $m$ и $M$ - соответственно минимальный и максимальньй индексы у $a_{i}$, действительно встречающиеся в $\bar{R}\left(a_{i}, b_{j}, \ldots\right)$. Тогда слово $\bar{R}\left(a_{i}, b_{j}, \ldots\right)$ начинается с $a_{0}$, т.е. имеем $m \leqslant 0 \leqslant M$.

Группа $G$ изоморфна группе

$$
\begin{aligned}
& G_{1}=\left\langle\left\langle t, a_{m}, \ldots, a_{M}, b_{j}, c_{j}, \ldots(j \in \mathbb{Z})\right| \bar{R}\left(a_{i}, b_{j}, \ldots\right)=1\right. \\
& \left.\left.\quad t a_{i}=a_{i+1} t(i=m, \ldots, M-1), t b_{j}=b_{j+1} t, t c_{j}=c_{j+1} t, \ldots(j \in \mathbb{Z})\right\rangle\right\rangle .
\end{aligned}
$$

Так как $\left|\bar{R}\left(a_{i}, b_{j}, \ldots\right)\right|<|R(t, a, b, c, \ldots)|$ и в $\bar{R}\left(a_{i}, b_{j}, \ldots\right)$ действительно входят буквы $a_{m}$ и $a_{M}$, то по индуктивному предположению элементы

$$
a_{m}, \ldots, a_{M-1}, b_{j}, c_{j}, \ldots \quad(j \in \mathbb{Z})
$$

так же как и элементы

$$
a_{m+1}, \ldots, a_{M}, b_{j}, c_{j}, \ldots \quad(j \in \mathbb{Z})
$$


суть свободные образующие порожденных ими подгрупп в группе

$$
\overline{G_{1}} \rightleftharpoons\left\langle\left\langle a_{m}, \ldots, a_{M}, b_{j}, c_{j}, \ldots(j \in \mathbb{Z}) \mid \bar{R}\left(a_{i}, b_{j}, \ldots\right)=1\right\rangle\right\rangle .
$$

Это означает, что $G_{1}$ есть HNN-расширение группы $\overline{G_{1}}$. Рассмотрим перевод $\bar{H}\left(b_{j}, c_{k}, \ldots\right)$ слова $H$ в алфавит $G_{1}$. Тогда $\bar{H}\left(b_{j}, c_{k}, \ldots\right)=1$ в $G_{1}$, а значит, и в $\overline{G_{1}}$. По индуктивному предположению это невозможно, так как в $\bar{R}\left(a_{i}, b_{j}, \ldots\right)$ входит буква $a_{0}$, которая не входит в слово $\bar{H}\left(b_{j}, c_{k}, \ldots\right)$.

Нам остается рассмотреть подслучай, когда в $H$ не входит буква $t$, т.е. $H=$ $H(a, b, c, \ldots)$ и $\bar{H}=H\left(a_{0}, b_{0}, c_{0}, \ldots\right)=1$ в $G_{1}$. Так как буква $t$ входит в $R$, то в слово $\bar{R}\left(a_{i}, b_{j}, \ldots\right)$ должна входить некоторая буква $a_{i}$ при $i \neq 0$. Но это опять противоречит индуктивному предположению.

Случай 2 . Все образующие, а их у нас по условию не менее 2 , входят в $R$ с ненулевой суммой показателей. Пусть буквы $t$ и $a$ входят в число образуюших. Допустим, что некоторое несократимое слово $H(t, b, c, \ldots)$, не содержащее $a$, равно 1 в $G$.

Обозначим $\beta \rightleftharpoons \sigma_{t}(R)$ и $\alpha \rightleftharpoons \sigma_{a}(R)$. Отображение $\Psi$, переводяшее $t$ в $x^{\alpha}, a$ в $y x^{-\beta}$, а остальные порождаюшие - в себя, задает гомоморфизм группы $G$ в группу

$$
G^{\prime} \rightleftharpoons\left\langle\left\langle x, y, b, c, \cdots \mid R\left(x^{\alpha}, y x^{-\beta}, b, c, \ldots\right)=1\right\rangle\right\rangle .
$$

Обозначим $R^{\prime}(x, y, b, c, \ldots) \rightleftharpoons R\left(x^{\alpha}, y x^{-\beta}, b, c, \ldots\right)$.

Буква $y$ существенно входит в слово $R^{\prime}(x, y, b, c, \ldots)$ и

$$
\sigma_{x}\left(R^{\prime}(x, y, b, c, \ldots)\right)=0
$$

Очевидно, из равенства $H(t, b, c, \ldots)=1$ в $G$ следует $H\left(x^{\alpha}, b, c, \ldots\right)=1$ в группе $G^{\prime}$ и, значит, $\sigma_{x}\left(H\left(x^{\alpha}, b, c, \ldots\right)\right)=0$. Так как при этом слово $H\left(x^{\alpha}, b, c, \ldots\right)$ несократимо и непусто, то в него входит некоторая буква, отличная от $x$.

Теперь, аналогично тому, как это вьше было сделано для группы $G$, группу $G^{\prime}$ изоморфно представим в виде $\mathrm{HNN}$-расширения группы $\overline{G_{1}^{\prime}}$ с соответствуюшим определяюшим соотношением вида $\overline{R^{\prime}}\left(y_{i}, b_{j}, c_{k}, \ldots\right)=1$, где $\left|\overline{R^{\prime}}\left(y_{i}, b_{j}, c_{k}, \ldots\right)\right|$ равно $|R(t, a, b, c, \ldots)|$ минус обшее число вхождений буквы $t$ в слово $R(t, a, b, c, \ldots)$. При этом и равенство $H\left(x^{\alpha}, b, c, \ldots\right)=1$ группы $G^{\prime}$ перейдет в нетривиальное равенство $\bar{H}\left(b_{j}, c_{k}, \ldots\right)=1$ в подгрупе $\overline{G_{1}^{\prime}}$. Но по индуктивному предположению это невозможно. Теорема 7.4 доказана.

В работе [89] Н. С. Романовский получил следующий интересный аналог теоремы В. Магнуса о свободе для групп, заданных более чем одним соотношением.

ТЕОРема 7.5. Если группа $G$ задана $n$ образующими и $m$ определяющими соотношениями, где $n>m$, то в ней найдутся $n-m$ образующих, которье порождают свободную подгруппу ранга $n-m$.

TеОРема 7.6. Существует единый алгоритм, решающий проблему равенства слов в любой конечно определенной группе с одним определяющим соотношениe⿻从. 
ДокАЗАТЕльство. Пусть дана конечно определенная группа

$$
G=\langle\langle a, b, c, \ldots, t \mid R(t, a, b, c, \ldots)=1\rangle\rangle .
$$

Можно считать, что все указанные в (10) буквы существенно входят в слово $R$ и оно циклически несократимо.

Будем искать алгоритм, проверяющий по данному собственному подалфавиту $\mathscr{A}$ и любому слову $X$, равно ли оно в $G$ какому-нибудь слову в алфавите $\mathscr{A}$, и указываюший такое слово, если ответ положительньй. Эту проблему, которую В. Магнус называл “обобшенной проблемой равенства”, мы будем называть ограниченной проблемой вхождения. Имея ответ на этот вопрос и используя теорему о свободе, можно будет решать и проблему равенства единице для данного слова $X$.

Доказательство будем вести индукцией по длине слова $R(t, a, b, c, \ldots)$. Можно считать, что $|R|>0$.

Случай 1. $\sigma_{t}(R)=0$.

Считаем, что слово $R$ начинается с $a$. Как и в доказательстве теоремы о свободе, запишем слово $R(t, a, b, c, \ldots)$ через новые образуюшие $a_{i}=t^{i} a t^{-i}, b_{j}=t^{j} b t^{-j}, \ldots$. Будем решать ограниченную проблему вхождения в новом задании той же группы

$$
\begin{aligned}
G=G_{0}= & \left\langle\left\langle t, a, b, c, \ldots, a_{m}, \ldots, a_{M}, b_{j}, c_{j}, \ldots(j \in \mathbb{Z})\right|\right. \\
& \bar{R}\left(a_{i}, b_{j}, \ldots\right)=1, a_{0}=a, b_{0}=b, \ldots, \\
& \left.\left.t a_{i}=a_{i+1} t(i=m, \ldots, M-1), t b_{j}=b_{j+1} t, t c_{j}=c_{j+1} t, \ldots(j \in \mathbb{Z})\right\rangle\right\rangle,
\end{aligned}
$$

где $m$ - минимальное, а $M$ - максимальное значение $i$, при котором множитель $a_{i}=$ $t^{i} a t^{-i}$ входит в полученное разложение $\bar{R}\left(t^{i} a t^{-i}, t^{j} b t^{-j}, \ldots\right)$ слова $R(t, a, b, c, \ldots)$. Так как слово $R$ начинается с $a$, то $m \leqslant 0 \leqslant M$.

Из теоремы о свободе следует, что $G_{0}$ является HNN-расширением групшы

$$
\begin{array}{r}
\overline{G_{0}}=\left\langle\left\langle a, b, c, \ldots, a_{m}, \ldots, a_{M}, b_{j}, c_{j}, \ldots\right.\right. \\
\left.\left.\qquad \bar{R}\left(a_{i}, b_{j}, \ldots\right)=1, a_{0}=a, b_{0}=b, \ldots\right\rangle\right\rangle .
\end{array}
$$

При любом целом $s$ через $R_{s}$ обозначим слово $t^{s} R t^{-s}$. Очевидно, соотношение $R_{s}=1$ равносильно соотношению $R=1$. Пусть $\overline{R_{s}}$ результат записи слова $R_{s}$ через $a_{i}=t^{i} a t^{-i}, b_{j}=t^{j} b t^{-j}, \ldots$ При любом $s$ слово $\overline{R_{s}}$ получается из $\bar{R}\left(a_{i}, b_{j}, \ldots\right)$ прибавлением числа $s$ к индексу каждой буквы, входящей в $\bar{R}$. При любом $r$ группы

$$
\begin{aligned}
& G_{r}=\left\langle\left\langle t, a_{m+r}, \ldots, a_{M+r}, b_{j}, c_{j}, \ldots(j \in \mathbb{Z})\right| \overline{R_{r}}\left(a_{i}, b_{j}, \ldots\right)=1,\right. \\
& \left.\left.t a_{i}=a_{i+1} t(i=m+r, \ldots, M+r-1), t b_{j}=b_{j+1} t, t c_{j}=c_{j+1} t, \ldots(j \in \mathbb{Z})\right\rangle\right\rangle
\end{aligned}
$$

и

$$
\overline{G_{r}}=\left\langle\left\langle a_{m+r}, \ldots, a_{M+r}, b_{j}, c_{j}, \ldots(j \in \mathbb{Z}) \mid \overline{R_{r}}\left(a_{i}, b_{j}, \ldots\right)=1\right\rangle\right\rangle
$$

изоморфны, соответственно, группам $G_{0}$ и $\overline{G_{0}}$.

При $k>0$ рассмотрим также группы

$$
\begin{aligned}
\Gamma_{r, k} & \rightleftharpoons\left\langle\left\langle t, a_{m+r}, \ldots, a_{M+r+k}, b_{j}, c_{j}, \ldots(j \in \mathbb{Z})\right| \overline{R_{s}}=1(s=r, r+1, \ldots, r+k),\right. \\
t a_{i} & \left.\left.=a_{i+1} t(i=m+r, \ldots, M-1+r+k), t b_{j}=b_{j+1} t, t c_{j}=c_{j+1} t, \ldots(j \in \mathbb{Z})\right\rangle\right\rangle
\end{aligned}
$$


и

$$
\overline{\Gamma_{r, k}} \rightleftharpoons\left\langle\left\langle a_{m+r}, \ldots, a_{M+r+k}, b_{j}, c_{j}, \ldots(j \in \mathbb{Z}) \mid \overline{R_{s}}=1(s=r, r+1, \ldots, r+k)\right\rangle\right\rangle .
$$

Очевидно, все групшы $\Gamma_{r, k}$ порождаются буквами $t, a_{0}, b_{0}, \ldots$, если $r \leqslant 0 \leqslant r+k$. Более того, все они изоморфны группе $G$. Группа $\Gamma_{r, 0}$ совпадает с группой $G_{r}$, которая также изоморфна $G$, а группа $\overline{\Gamma_{r, 0}}$ совпадает с группой $\overline{G_{r}}$.

При $k=1$ группа $\overline{\Gamma_{r, k}}$ есть свободное произведение двух групп $\overline{G_{r}}$ и $\overline{G_{r+1}}$ с объединенной свободной подгруппой, порожденной в них всеми образуюшими, кроме одной из букв $a_{i}$ (точнее, кроме $a_{m+r}$ и $a_{M+r+1}$, соответственно).

В обшем случае $\overline{\Gamma_{r, k}}$ есть свободное произведение группы $\overline{\Gamma_{r, k-1}}$ и групшы $\overline{G_{k}} \mathrm{c}$ соответствуюшей объединенной подгруппой. Так как при любых $r$ и $k$ группы $\overline{\Gamma_{r, k-1}}$ и $\overline{\Gamma_{r+1, k-1}}$ изоморфны, то группа $\Gamma_{r, k}$ есть HNN-расширение группы $\overline{\Gamma_{r, k}}$.

Очевидно, $\left|\overline{R_{s}}\right|=|\bar{R}|<|R|$. По индуктивному предположению ограниченная проблема вхождения разрешима во всех группах $\overline{G_{r}}$. Отсюда индукцией по $k$ на основании теоремы 7.3 получим, что ограниченная проблема вхождения разрешима во всех группах $\overline{\Gamma_{r, k}}$.

Теперь перейдем к решению ограниченной проблемы вхождения для группы $G$. В силу теоремы о свободе достаточно для групшы $G$ решить проблему вхождения в подгруппу $F$, порожденную всеми образующими кроме одной.

Подслучай 1.1. Подгруппа $F$ порождается в $G$ всеми буквами, кроме $t$.

Рассмотрим произвольное слово $X$ группы $G$. Если $\sigma_{t}(X) \neq 0$, то $X \notin F$. Пусть $\sigma_{t}(X)=0$. Записав слово $X(t, a, b, c, \ldots)$ через $t^{i} a t^{-i}, t^{j} b t^{-j}, \ldots$, получим $X=\bar{X}\left(t^{i} a t^{-i}, t^{j} b t^{-j}, \ldots\right)$. Пусть $m_{1}$ - минимальное, а $M_{1}$ - максимальное значение $i$, при котором $t^{i} a t^{-i}$ входит в запись слова $\bar{X}$. Положим $m_{2} \rightleftharpoons \min \left(m, m_{1}\right)$ и $M_{2} \rightleftharpoons \max \left(M, M_{1}\right)$.

Так как в задании групп $\Gamma_{r, k}$ и $\overline{\Gamma_{r, k}}$ при $r \rightleftharpoons m_{2}-m$ и $k \rightleftharpoons M_{2}-m_{2}-M+m$ участвуют все буквы $a_{i}$ при $m_{2} \leqslant i \leqslant M_{2}$ и $\overline{\Gamma_{r, k}}$ есть подгруппа групшы $\Gamma_{r, k}$, которая изоморфна $G$, то нам достаточно решить вопрос о вхождении слова $\bar{X}\left(a_{i}, b_{j}, \ldots\right)$ в подгруппу группы $\overline{\Gamma_{r, k}}$, порожденную буквами $a_{0}, b_{0}, \ldots$ А это было сделано выше.

Подслучай 1.2. Подгруппа $F$ порождается в $G$ всеми буквами, кроме $a$.

Переходя к слову $X t^{-\sigma_{t}(X)}$, мы можем считать, что $\sigma_{t}(X)=0$. Тогда можем рассмотреть представление $\bar{X}\left(a_{i}, b_{j}, \ldots\right)$, для которого нужно проверить принадлежность подгрупп, порожденной всеми буквами, кроме всех букв $a_{i}$, в групе $\Gamma_{r, k}$, алфавит которой содержит все буквы $a_{i}$ из $\bar{X}$ и $\bar{R}$. А это следует из разрешимости ограниченной проблемы вхождения для групп $\overline{\Gamma_{r, k}}$.

Случай 2. Все образующие входят в $R(t, a, b, c, \ldots)$ с ненулевой суммой показателей. Если в $R$ входит всего одна буква, то утверждение очевидно. Пусть буквы $a$ и $t$ входят в $R$, причем $\sigma_{a}(R)=\beta$ и $\sigma_{t}(R)=\alpha$. В силу равнозначности букв алфавита группы $G$ в рассматриваемом случае, достаточно решить проблему вхождения в подгруппу $F$, порожденную всеми буквами, кроме $t$.

Пусть $R^{\prime}(x, y, b, c, \ldots)$ есть результат подстановки $t=y x^{-\beta}$ и $a=x^{\alpha}$ в слово $R(t, a, b, c, \ldots)$. Очевидно, $\sigma_{x}\left(R^{\prime}\right)=0$. Рассмотрим группу

$$
G^{\prime} \rightleftharpoons\left\langle\left\langle x, b, c, \ldots, y \mid R^{\prime}=R\left(y x^{-\beta}, x^{\alpha}, b, c, \ldots\right)=1\right\rangle\right\rangle
$$


Слово $R^{\prime}$ может быть длиннее, чем $R$, но, применив к нему кодирование из случая 1 , мы перейдем к соотношению $\overline{R^{\prime}}\left(y_{i}, b_{j}, \ldots\right)=1$, которое короче, чем исходное соотношение $R=1$. Так как на основании индуктивного предположения и в силу доказанного в рассмотренном вьше случае 1 мы имеем разрешимость ограниченной проблемы вхождения для соотношения

$$
R\left(y x^{-\beta}, x^{\alpha}, b, c, \ldots\right)=1,
$$

то нам остается свести проблему вхождения для группы $G$ к проблеме вхождения для групшы $G^{\prime}$.

Наряду с группами $G$ и $G^{\prime}$ рассмотрим еше группу

$$
G^{\prime \prime} \rightleftharpoons\left\langle\left\langle t, x, b, c, \cdots \mid R\left(t, x^{\alpha}, b, c, \ldots\right)=1\right\rangle\right\rangle .
$$

Очевидно, для любого слова $X(t, a, b, \ldots)$ из группы $G$ имеем

$$
X(t, a, b, \ldots) \in F \Longleftrightarrow X\left(t, x^{\alpha}, b, \ldots\right) \text { равно в групше } G^{\prime \prime}
$$

$$
\text { некоторому слову } \Psi\left(x^{\alpha}, b, \ldots\right)
$$

и

$$
X\left(t, x^{\alpha}, b, \ldots\right)=\Psi\left(x^{\alpha}, b, \ldots\right) \text { в } G^{\prime \prime} \Longleftrightarrow X\left(y x^{-\beta}, x^{\alpha}, b, \ldots\right)=\Psi\left(x^{\alpha}, b, \ldots\right) \text { в } G^{\prime} .
$$

Для проверки сушествования такого $\Psi$, для которого выполняется последнее равенство, сначала выясним, существует ли такое $\Phi(x, b, \ldots)$ (без вхождений буквы $y$ ), что $X\left(y x^{-\beta}, x^{\alpha}, b, \ldots\right)=\Phi(x, b, \ldots)$ в $G^{\prime}$. Если такого $\Phi(x, b, \ldots)$ нет, то $X \notin F$. Если такое $\Phi(x, b, \ldots)$ найдется, то в силу теоремы о свободе нам остается проверить, равно ли оно в свободной группе, порожденной в $G^{\prime}$ буквами $x, b, \ldots$, некоторому слову вида $\Psi\left(x^{\alpha}, b, \ldots\right)$. Теорема 7.6 доказана.

Как уже отмечалось выше, разрешимость проблемы тождества для групп с одним определяюшим соотношением была установлена В. Магнусом еше в 1932 году в работе [50]. Вопрос о сушествовании групп с неразрешимой проблемой равенства слов, заданных $k$ определяюшими соотношениями, при $2 \leqslant k \leqslant 11$ остается открытым.

СлЕДСТВИЕ 7.6.1. Если при $i=1,2, \ldots, k$ ииклически несократимое слово $R_{i}\left(x_{i}, y_{1}, \ldots, y_{n}\right)$ содержит букву $x_{i}$, то в группе

$$
\left\langle\left\langle x_{1}, \ldots, x_{k}, y_{1}, \ldots, y_{n} \mid R_{1}\left(x_{1}, y_{1}, \ldots, y_{n}\right)=1, \ldots, R_{k}\left(x_{k}, y_{1}, \ldots, y_{n}\right)=1\right\rangle\right\rangle
$$

разрешима проблема равенства.

СлЕДСТвиЕ 7.6.2. Проблема равенства разрешима в любой группе, имеющей задание

$$
\begin{array}{r}
\left\langle\left\langle a_{1}, \ldots, a_{n}, b_{1}, \ldots, b_{m}\right| R_{1}\left(a_{1}, \ldots, a_{n}\right)=1, R_{2}\left(b_{1}, \ldots, b_{m}\right)=1,\right. \\
\left.\left.U\left(a_{1}, \ldots, a_{n-1}\right)=V\left(b_{1}, \ldots, b_{m-1}\right)\right\rangle\right\rangle
\end{array}
$$

где буквы $a_{n}$ и $b_{m}$ входят в ичклически несократимые слова $R_{1}\left(a_{1}, \ldots, a_{n}\right)$ и $R_{2}\left(b_{1}, \ldots, b_{m}\right)$, а $U\left(a_{1}, \ldots, a_{n-1}\right)$ и $V\left(b_{1}, \ldots, b_{m-1}\right)$ - непустые несократимые слова.

Заметим, что проблема сопряженности для групा, заданных одним определяющим соотношением, остается пока открытой. Ее решение найдено только для определяющих определяюших соотношений вида $F^{k}=1$, где $k>1$ [68]. 


\section{8. Проблемы распознавания групповых свойств}

Из результата П. С. Новикова онеразрешимости проблемы равенства слов нетрудно вьвести неразрешимость проблемы изоморфизма, поставленной Тице еше в 1908 году [102] и включенной М. Дэном [31] в число фундаментальных алгоритмических проблем для конечно определенных групп.

ОБШАЯ ПРОБЛЕМА ИЗОМОРФИЗМА. Найти алгоритм, который бъ nо любъм двум заданным конечно определенным группам определял, изоморфны они или нет.

ЧАСТНАЯ ПРОБЛЕМА ИЗОМОРФИЗМА. Для фиксированной группы $F_{0}$ найти алгоритм, который бъ по любой конечно определенной группе $F$ проверял, изоморфна она әруппе $F_{0}$ или нет.

Приведем простое доказательство неразрешимости частной проблемы изоморфизма для случая, когда $F_{0}$ - свободная группа. Тем самым будет установлена и неразрешимость общей проблемы изоморфизма.

Пусть

$$
G=\left\langle\left\langle a_{1}, a_{2}, \ldots, a_{m} \mid R\right\rangle\right\rangle
$$

есть группа с неразрешимой проблемой равенства слов. Можно считать, что все образуюшие $a_{i}$ имеют в группе $G$ бесконечный порядок. Кроме того, можно считать, что в группе $G$ выделен специальньй класс слов $\mathfrak{M}$, такой, что невозможен алгоритм, проверяющий равенство $X=1$ в группе $G$ уже для слов $X$ из $\mathfrak{M}$, и при этом для каждого $X$ из множества $\mathfrak{M}$ элемент $[X]$ либо равен 1 , либо имеет бесконечный порядок в группе $G$. В частности, этим свойством обладает в группе $G$ множество слов вида $k^{-1}\left(X^{-1} t X\right) k\left(X^{-1} t^{-1} X\right)$, рассмотренное вьше в теореме 6.6.

Каждому такому слову $A \rightleftharpoons k^{-1}\left(X^{-1} t X\right) k\left(X^{-1} t^{-1} X\right)$ групшы $G$ поставим в соответствие конечно определенную группу $G_{A}$, заданную образуюшими

$$
a_{1}, a_{2}, \ldots, a_{m}, q_{1}, q_{2}, \ldots, q_{m}
$$

и определяюшими соотношениями

$$
R \cup\left\{a_{i} q_{i}=q_{i} A \mid 1 \leqslant i \leqslant m\right\} .
$$

Если $A=1$ в группе $G$, то из определяюших соотношений (2) следует, что в групе $G_{A}$ выполняется равенство $a_{i}=1$ при любом $i=1,2, \ldots, m$. Поэтому группа $G_{A}$ изоморфна свободной группе $F_{m}$ с $m$ свободньми образуюшими.

Если же $A \neq 1$ в групше $G$, то по условию элемент $A$ имеет бесконечный порядок в основании $G$ группы $G_{A}$ по системе проходных букв

$$
q_{1}, q_{2}, \ldots, q_{m}
$$

Все образующие $a_{i}$ также имеют бесконечньй порядок в группе $G$. Следовательно, при $A \neq 1$ в групше $G$ буквы (3) образуют правильную систему проходных букв. Тогда $G$ есть подгруппа группы $G_{A}$, так что в этом случае группа $G_{A}$ не может быть изоморфна свободной группе $F_{m}$. 
Из доказанного в силу неразрешимости проблемы равенства слов для группы $G$ следует, что невозможен алгоритм, проверяющий изоморфизм данной свободной группе $F_{m}$ уже для групп из класса

$$
\left\{G_{A} \mid A \in G\right\}
$$

В 1955 году [2] было доказано, что частная проблема изоморфизма неразрешима для каждой конечно определенной группы $F_{0}$. Принципиальную трудность здесь представлял случай, когда $F_{0}$ есть единичная группа. Дело в том, что группа, содержащая проходные буквы, всегда имеет свободную подгруппу и потому не может быть единичной. ${ }^{1}$ В работе [2], полное изложение которой опубликовано в [3], впервые было построено семейство групп (4) с параметром $A$, пробегаюшим множество слов данной группы $G$ с неразрешимой проблемой равенства, для которого выполнено условие:

группа $G_{\text {А }}$ единична в том и только том случае, если слово А равно 1 в zpynne $G$.

Вскоре А. А. Марков (см. [59]), опираясь на эту конструкцию, построил такое бесконечное семейство 4-мерных многообразий $M_{A}$ с фундаментальными группами $G_{A}$, что $M_{A}$ гомеоморфно фиксированному многообразию в том и только том случае, когда группа $G_{A}$ единичная. Тем самым была доказана неразрешимость известной алгоритмической проблемы топологии - проблемы распознавания гомеоморфизма многообразий размерности $n$ при $n \geqslant 4$, сформулированной М. Дэном еще в 1909 году. Позже С.П. Новиков доказал неразрешимость для $n=5$ поставленной в начале века проблемы А. Пуанкаре об алгоритмическом распознавании сферы среди $n$-мерных многообразий [108]. Отметим, что для $n=4$ эта проблема остается открытой. Сравнительно недавно А. Томпсон [100] доказала, что проблема распознавания 3-мерной сферы решается положительно. Последнему результату предшествовала некоторая предыстория, в ходе которой определенный вклад в решение этого вопроса внесли также Хакен и Рубинштейн (см. [62]).

Здесь мы приведем предложенньй недавно Ч. Миллером III [65] весьма простой способ построения семейства групп $G_{A}$ с 2 образуюшими с неразрешимой проблемой распознавания единичности.

ЛЕмма 8.1 (лемма Миллера). Пусть (1) есть произвольная конечно определенная группа, а $w$ - любое слово в ее алфавите. Обозначим через $G_{w}$ группу со следующим заданием:

$$
\begin{aligned}
& \left\langle\left\langle a_{1}, a_{2}, \ldots, a_{m}, a, b, c,\right| R, a^{-1} b a=c^{-1} b^{-1} c b c, a^{-2} b^{-1} a b a^{2}=c^{-2} b^{-1} c b c^{2},\right. \\
& \left.\left.a^{-3}[w, b] a^{3}=c^{-3} b c^{3}, a^{-(3+i)} a_{i} b a^{(3+i)}=c^{-(3+i)} b c^{(3+i)}, i=1,2, \ldots, m\right\rangle\right\rangle,
\end{aligned}
$$

где $а$, , и и - новие буквй.

Группа $G_{w}$ порождается двумя әлементами $b$ и $с a^{-1}$.

Eсли $w=1$ в группе $G$, mо $G_{w}$ - единичная группа.

Eсли $w \neq 1$ в группе $G$, mo $G-$ nодгруппа группь $G_{w}$.

\footnotetext{
${ }^{1}$ Заметим, что в известной монограффии А. Г. Куроша, изданной в 1953 году [45; с. 266], проблема изоморфизма упомянута в следующем контексте: "Эта проблема не имеет пока решения даже в том случае, когда одна из заданных групп является единичной".
} 
ДокАЗАТЕльство. Очевидно, первое определяюшее соотношение групшы $G_{w}$ можно записать в виде $c=b\left(c a^{-1}\right) b\left(c a^{-1}\right)^{-1} b^{-1}$, а последняя серия соотношений позволяет выразить все порождаюшие $a_{i}$ через $a, b, c$, что завершает доказательство первого утверждения леммы.

Пусть $w=1$ в группе $G$. Тогда из соответствуюших соотношений получаем сначала $b=1$, затем $c=1$ и $a=1$. Следовательно, группа $G_{w}$ - единичная.

Пусть $w \neq 1$ в группе $G$. В свободной группе с порождаюшими $d$ и $c$ элементы $d$, $c^{-1} d^{-1} c d c, c^{-2} d^{-1} c d c^{2}, c^{-3} b c^{3}, c^{-(3+i)} d c^{(3+i)}(i=1,2, \ldots, m)$ являются свободными образующими порожденной ими подгруппы $H_{1}$.

Аналогично в свободном произведении $G *\langle\langle a, b\rangle\rangle$, элементы $b, a^{-1} b a, a^{-2} b^{-1} a b a^{2}$, $a^{-3}[w, b] a^{3}, a^{-(3+i)} a_{i} b a^{(3+i)}(i=1,2, \ldots, m)$ также являются свободными образующими порожденной ими подгруппы $H_{2}$.

Группа $G_{w}$ является свободным произведением групп $G *\langle\langle a, b\rangle\rangle$ и $\langle\langle d, c\rangle\rangle$ с объединением по подгруппам $H_{1}$ и $H_{2}$. Следовательно, $G$ - подгруппа группы $G_{w}$.

Из этой леммы Миллера легко следует

ЛЕмма 8.2. Каждая счетная группа $G$ вложима в 2-порожденную группу $H$. Если при этом исходная группа $G$ бъла рекурсивно определенной (или конечно определенной), то и расширение $H$ будет такой жсе группой.

Применив ту же конструкцию к группе с неразрешимой проблемой равенства слов, получим конечно определенную группу с двумя порождаюшими, имеющую неразрешимую проблему равенства.

ТЕОРема 8.1. Проблема изоморфизма единичной группе неразрешима уже в классе 2-порожденньх групп. Частная проблема изоморфизма для каждой т-порожсденой группы неразрешима в классе $(m+2)$-порожденных групп.

ДоказАТЕльство. Для доказательства первого утверждения достаточно в лемме Миллера взять в качестве исходной группы $G$ конечно определенную группу с неразрешимой проблемой равенства. Очевидно, в классе циклических групп проблема изоморфизма решается положительно.

Для доказательства второго утверждения теоремы для данной конечно определенной группы $F_{0}$ с $m$ порождаюшими рассмотрим семейство групп

$$
G_{w}^{\prime} \rightleftharpoons G_{w} * F_{0}
$$

где $w$ пробегает множество всех слов в группе $G$ с неразрешимой проблемой равенства. Если $w=1$ в группе $G$, то группа $G_{w}^{\prime}$ изоморфнна группе $F_{0}$, а при $w \neq 1$ ранг групшы $G_{w}^{\prime}$ больше ранга группы $F_{0}$ (см. следствие IV.1.9 в [47]).

В работах [2], [3] были доказаны также несколько теорем о неразрешимости проблем распознавания различных инвариантных групповых свойств. Под проблемой распознавания данного группового свойства $\alpha$ здесь понимается задача нахождения алгоритма, распознающего по любой конечно определенной группе, вьполнено или нет в ней данное свойство. Групповое свойство называется инвариантнылм, если оно, будучи выполнено в некоторой группе $G$, выполняется также во всех изоморфных ей группах. Инвариантное свойство называется наследственным, если оно, будучи выполнено в некоторой группе $G$, вьполняется также во всех ее подгруппах. 
Наследственное групповое свойство называется нетривиальным, если существует группа, обладающая этим свойством, и группа, не обладающая им. В работе [3] было впервые доказано, что для произвольного нетривиального наследственного свойства $\alpha$ и произвольного инвариантного свойства $\beta$ невозможен алгоритм распознавания конъюнкции свойств $\alpha$ и $\beta$. Очевидно, здесь условие нетривиальности свойства $\alpha$ необходимо. Заметим, что утверждение остается в силе, если в нем опустить свойство $\beta$. Из этих двух утверждений уже следует, что для большинства наиболее интересных групповых свойств проблема распознавания неразрешима. В частности, наследственным является любое тож дественное соотношение, например - абелевость, нильпотентность, разрешимость и др. Сюда относятся также и такие свойства, как тривиальность, конечность, периодичность, быть группой без кручения, цикличность, быть свободной группой, иметь разрешимую проблему равенства и т. д.

Впоследствии в работах С.И. Адяна [5], [6] и М. О. Рабина [85] была получена более общая теорема о невозможности распознавания инвариантных групповых свойств, аналогичная уже известной тогда теореме А. А. Маркова о распознавании свойств полугрупп [58].

Инвариантное групповое свойство $\alpha$ назьвается марковским, если существует конечно определенная группа со свойством $\alpha$ и конечно определенная группа, не вложимая ни в какую конечно определенную группу со свойством $\alpha$. В указанных работах С. И. Адяна и М. О. Рабина было доказано, что для каждого марковского группового свойства $\alpha$ невозможен алгоритм, распознающий по любой конечно определенной группе, выполнено в ней свойство $\alpha$ или нет. Если предложенное С. И. Адяном доказательство использовало технику его первоначальной работы 1955 года, то М. О. Рабин внес существенное упрощение в конструкцию семейства груп $G_{A}$, используя свободные произведения групп с объединенной подгруппой.

Теорема 8.2 (теорема Адяна-Рабина). Любое марковское групповое свойство алгоритмически нераспознаваемо.

ДокАЗАТЕльство. Пусть $\alpha-$ произвольное марковское групповое свойство, т.е. оно инвариантно и существуют как конечно определенная группа $G^{+}$со свойством $\alpha$, так и конечно определенная группа $G^{-}$, которую нельзя вложить ни в какую конечно определенную группу со свойством $\alpha$. Кроме того, пусть $H$ - конечно определенная группа с неразрешимой проблемой равенства слов. Для произвольного слова $w$ в алфавите групшы $H$, применяя конструкцию Миллера, строим конечно определенную группу $\left(G^{-} * H\right)_{w}$. Тогда имеет место логическая эквивалентность:

$$
w=1 \text { в группе } H \Longleftrightarrow \text { группа }\left(G^{-} * H\right)_{w} * G^{+} \text {обладает свойством } \alpha \text {. }
$$

Следовательно, невозможен алгоритм распознавания свойства $\alpha$ для всех конечно определенных групп.

Из приведенной теоремы Адяна-Рабина вытекает одно любопытное следствие. Пусть $K_{\alpha}$ есть класс всех групп, обладающих инвариантным свойством $\alpha$. С каждым таким классом $K_{\alpha}$ можно связать две алгоритмические проблемы: проблема равенства слов для групп из класса $K_{\alpha}$ и проблема распознавания по любой конечно определенной группе, принадлежит ли она классу $K_{\alpha}$. Оказывается, что для любого непустого класса $K_{\alpha}$ по крайней мере одна из этих двух проблем неразрешима, т.е. здесь имеет место своего рода "приниип неопределенности". 
Каждое нетривиальное наследственное свойство является марковским. Свойство “быть простой группой" дает пример марковского, но не наследственного свойства. Сушествование конечно определенной группы, которая не вложима ни в какую конечно определенную простую группу, следует из разрешимости проблемы равенства для любой конечно определенной простой группы. Если группа единичная, то проблемы равенства для нее не сушествует. Алгоритм распознавания равенства слов для всех неединичных простых групп составляется из следуюших двух параллельных алгоритмических процессов для данной конечно определенной группы $H$ и произвольного слова $A$ в его алфавите: первьй последовательно перечисляет все слова, равные слову $A$ в группе $H$, и останавливается, когда найдет среди них пустое слово; а второй процесс перечисляет все слова, равные 1 в группе $H^{\prime}$, полученной добавлением к $H$ нового соотношения $A=1$, и останавливается только в том случае, если будет установлено, что в $H^{\prime}$ все образующие равны 1 . Очевидно, для неединичной простой группы $H$ один и только один из указанных процессов завершится в конечное число шагов и даст ответ на вопрос, равно слово $A$ единище в группе $H$ или нет.

Как было отмечено в работе [3], теорему о нераспознаваемости марковских групповых свойств нельзя распространить на любые нетривиальные инвариантные групповые свойства. Так свойство “совпадать со своим коммутантом" является нетривиальным и алгоритмически распознаваемо, так как можно воспользоваться алгоритмом распознавания единичности для абелевых групп.

Описанная вьше конструкция позволяет доказать алгоритмическую нераспознаваемость и для некоторых свойств, не являюшихся марковскими. Например, свойство быть 2-порожденной группой не является марковским. В то же время это свойство алгоритмически нераспознаваемо, что также легко вытекает из леммы Миллера, если в рассмотренной нами конструкции взять в качестве группы $G^{-}-$единичную группу, а в качестве групшы $G^{+}$- свободную группу ранга 2.

Р. Д. Павлов [82] на основе введенного С. И. Адяном в работе [3] понятия квазипроходной буквы получил усиление теоремы Адяна-Рабина, показав, что проблема распознавания произвольного марковского свойства $\alpha$, выполняющегося для некоторой конечно определенной групш с $n$ образующими, неразрешима в классе конечно определенных групп с $n+1$ образуюшим. Им же была показана неразрешимость проблемы распознавания изоморфизма свободной группе ранга $n$ в классе конечно определенных групп с $n+1$ образующим. Очевидно, проблема распознавания изоморфизма свободной группе ранга $n$ в классе конечно определенных групп с $n$ образуюшими разрешима.

Для класса всех конечно определенных абелевых групп легко доказывается разрешимость как проблемы равенства слов, так и проблемы изоморфизма. Среди наиболее интересных положительных результатов, связанных с проблемой изоморфизма, следует отметить совместную работу $\Phi$. Грюневальда и Д. Сигала [37] с решением проблемы изоморфизма для класса всех конечно определенных нильпотентных групп, а также решение М.А. Тайцлиным [97] проблемы изоморфизма для класса конечно определенных коммутативных полугруп, которая тоже оставалась открытой в течение длительного времени.

\section{9. Проблема вхождения и некоторые результаты о вложении}

Проблема вхождения в данную подгруппу $H$ группы $G$ формулируется следуюшим образом. 
Найти алгоритм, который бъ по любому әлементу $g$ группь $G$ определял принадлежст он подгруппе $H$ или нет.

Общая проблема вхождения для данной групшы $G$ заключается в отыскании единого алгоритма, решающего эту проблему для любой подгруппы. Обычно порождаюшие элементы подгруппы $H$ и элемент $g$ задаются словами в алфавите группы $G$.

Очевидно, проблема равенства слов для групшы $G$ равносильна проблеме вхождения в ее единичную подгруппу. Важный частньй случай проблемы вхождения получается, если ограничиться рассмотрением лишш таких подгрупп $H$, которые порождаются некоторыми образуюшими исходной группы. Этот ограниченньй вариант проблемы вхождения В. Магнус назьвал расширенной проблемой равенства слов. Вьше мы привели полученное В. Магнусом решение этой проблемы для групп с одним определяюшим соотношением. Вопрос о разрешимости проблемы вхождения для групп с одним определяюшим соотношением в обшем случае пока остается открытым.

Еще в 1921 году Е. Нильсен [69] указал алгоритм, решающий проблему вхождения в любую конечно порожденную подгруппу произвольной свободной групшы. Этот результат был обобшен К.А. Михайловой, доказавшей разрешимость проблемы вхождения для свободного произведения любых двух групп с разрешимой проблемой вхождения [64]. Позже В. Н. Безверхний доказал разрешимость проблемы вхождения для свободного произведения двух свободных групп с объединением по циклическим подгруппам [19]. Он же доказал разрешимость проблемы вхождения для групп с одним определяющим соотношением, имеющих нетривиальный центр.

А.И. Мальцев [55] доказал разрешимость проблемы вхождения для конечно порожденных нильпотентных групп, а Н. С. Романовский [88] решил положительно проблему вхождения для любой конечно порожденной метабелевой группы.

Приведем доказательство результата К.А. Михайловой [63] о неразрешимости проблемы вхождения для прямого произведения двух свободных групп ранга 2 и некоторые интересные следствия, получаемые из него.

ТЕОРема 9.1. В прямом произведении двух свободных групп ранга 2

$$
F_{2} \times F_{2}=\left\langle\left\langle a_{1}, a_{2}, b_{1}, b_{2} \mid a_{i} b_{j}=b_{j} a_{i}(1 \leqslant i, j \leqslant 2)\right\rangle\right\rangle
$$

можно указать конечно порохсденую подгруппу, для которой проблема вхождения неразрешима

ДокАЗАТЕльство. Рассмотрим 2-порожденную группу с неразрешимой проблемой равенства слов

$$
G=\left\langle\left\langle a_{1}, a_{2} \mid R_{1}=1, \ldots, R_{m}=1\right\rangle\right\rangle
$$

Через $H_{G}$ обозначим подгруппу групшы $F_{2} \times F_{2}$, порожденную элементами

$$
a_{1} b_{1}, a_{2} b_{2},\left\{R_{i} \mid i=1, \ldots, m\right\}
$$

Нам достаточно доказать, что слово $A$ в алфавите группь $G$ равно единице в $G$ в том и только том случае, когда $A \in H_{G}$.

Для данного слова $E$ в алфавите из $a$-букв через $B_{E}$ обозначим результат замены в $E$ каждой буквы $a_{j}$ буквой $b_{j}$. Очевидно, для любого слова $E$ в алфавите из 
$a$-букв слово $E B_{E}$ принадлежит $H_{G}$. Отсюда следует, что в $H_{G}$ лежат все слова вида $E R_{i} E^{-1}$, а в силу леммы 5.1 - и все слова, равные 1 в $G$.

Пусть некоторьй элемент группы (1), представимьй словом $A$ из $a$-букв, принадлежит подгруппе $H_{G}$, т.е. в группе (1) выполнено равенство

$$
A=H_{1} H_{2} \ldots H_{k}
$$

где $H_{i}$ суть какие-то порождаюшие (3) или им обратные. Очевидно, сумма показателей $b$-букв в слове $W \rightleftharpoons H_{1} H_{2} \ldots H_{k}$ равна 0 . Равенство $A=1$ в $G$ доказывается индукцией по числу вхождений в $W$ множителей вида $R_{i}^{ \pm 1}$. Пусть $H_{t}$ - последний такой множитель в записи (4), а $W_{1}$ - результат вычеркивания из $W$ этого множителя $H_{t}$. В группе (1) выполнены равенства

$$
W=W_{1}\left(H_{t+1} \ldots H_{k}\right)^{-1} H_{t} H_{t+1} \ldots H_{k}=W_{1} E R_{i}^{ \pm 1} E^{-1}=A_{1} E R_{i}^{ \pm 1} E^{-1}
$$

где $A_{1}$ и $E$ суть проекции слов $W_{1}$ и $H_{t+1} \ldots H_{k}$ на алфавит из $a$-букв. Остается заметить, что по индуктивному предположению $A_{1}=1$ в $G$.

Из доказанной теоремы следует неразрешимость проблемы вхождения для любой группы, в которую вкладывается группа $F_{2} \times F_{2}$. В частности, так как свободная группа $F_{2}$ ранга 2 вложима в группу $S L(2, \mathbb{Z})$ целочисленных матрищ второго порядка с равньм 1 определителем, то группа $S L(4, \mathbb{Z})$ целочисленных матриц порядка 4 с равным 1 определителем имеет неразрешимую проблему вхождения. Другими словами, можно указать такое конечное множество матрищ четвертого порядка

$$
\left\{M_{1}, M_{2}, \ldots, M_{n}\right\}
$$

что невозможен алгоритм, определяющий по произвольной матрице $M$ четвертого порядка, можно ли ее представить в виде произведения матриц из множества (5) и им обратных. Это замечание о неразрешимости проблемы распознавания представимости матрищ порядка 4 было впервые отмечено А. А. Марковьм [60].

Приведем еще одно интересное следствие теоремы К. А. Михайловой, которое было доказано А. А. Чеботарем в работе [28].

Группа

$$
G^{*}=\langle\langle a, b, c, d \mid a c=c a, b c=c b, a d=d a, b d=d b, a t=t c, b t=t d, c t=t b\rangle\rangle
$$

есть HNN-расширение группы $F_{2} \times F_{2}$ с образуюшими $a, b, c, d$, так как соответствие

$$
\varphi(a) \rightleftharpoons c, \quad \varphi(b) \rightleftharpoons d, \quad \varphi(c) \rightleftharpoons b
$$

определяет изоморфное отображение подгруппы, порожденной в $F_{2} \times F_{2}$ элементами $a, b, c$, в подгруппу, порожденную элементами $c, d, b$. Следовательно, $F_{2} \times F_{2}$ есть подгруппа групшы $G^{*}$.

Используя преобразования Тице, нетрудно показать, что группа $G^{*}$ имеет следующее задание:

$$
\left\langle\left\langle a, t \mid a t^{-1} a t=t^{-1} a t a, a t^{-3} a t^{3}=t^{-3} a t^{3} a\right\rangle\right\rangle .
$$


Таким образом, мы получаем пример группы с 2 определяюшими соотношениями, которая имеет неразрешимую проблему вхождения. Это один из немногих известных в настоящее время примеров неразрешимых алгоритмических проблем для групп с двумя определяюшими соотношениями. В той же работе [28] было доказано, что группа $F_{2} \times F_{2}$ не вложима в группы с одним определяющим соотношением.

T. А. Маканина [54] показала, что группу $F_{2} \times F_{2}$ можно вложить в группу кос с $n$ нитями $\mathbf{B}_{n}$ при $n \geqslant 5$. Поэтому для групш $\mathbf{B}_{n}$ также неразрешима проблема вхождения. Открытым остается вопрос о разрешимости проблемы вхождения для групшы кос $\mathbf{B}_{4}$.

Счетную группу $G$ будем называть рекурсивно определенной, если она задается рекурсивно перечислимым множеством определяюших соотношений при некотором наборе порождающих. Очевидно, рекурсивная определенность группы $G$ равносильна рекурсивной перечислимости множества всех слов, равных 1 в этой группе.

Заметим, что группа, заданная рекурсивно перечислимым множеством определяющих соотношений

$$
R_{1}=1, R_{2}=1, \ldots, R_{i}=1, \ldots,
$$

может быть задана в том же алфавите и множеством соотношений вида

$$
R_{1}=1, R_{1} R_{2}=1, \ldots, R_{1} R_{2} R_{3} \ldots R_{i}=1, \ldots
$$

которое будет не только рекурсивно перечислимо, но и рекурсивно, так как длины этих соотношений растут монотонно.

Г. Хигман в работе [39] доказал следуюшую интересную теорему.

ТЕОРема ХИГмана. Конечно порожденная группа вложима в некоторую конечно определенную группу тогда и только тогда, когда она является рекурсивно определенной.

Доказательство. Следуя работе С. Аандераа [1], здесь мы используем ту же конструкцию В. Буна, которая была использована вьше при доказательстве теоремы 6.5.

Если группа $G$ вложена в некоторую конечно определенную групшу $F$, то множество слов подгрупшы $G$, равных 1 в группе $F$, рекурсивно перечислимо, т.е. $G$ является рекурсивно определенной группой.

Пусть конечно порожденная группа $G$ имеет рекурсивно перечислимое множество определяюших соотношений. Пусть

$$
\left\{b_{1}, b_{2}, \ldots, b_{p}\right\}
$$

- множество ее полугрупповых образуюших, а $E$ - множество всех слов, равных 1 в $G$.

Рекурсивная перечислимость множества $E$ означает, что существует машина Тьюринга $T_{E}$ c начальным состоянием $q_{1}$ и заключительным $-q_{0}$, внешший алфавит которой содержит алфавит (6), такал, что для произвольного слова $X$ в алфавите (6) вьполнено условие

начав работать в конфигурации $q_{1} X$, машина $T_{E}$ остановится $\Longleftrightarrow X \in E$. 
Пусть $S_{T_{E}}=\left\{s_{0}, s_{1}, \ldots, s_{N}\right\}$ - внешний алфавит, а $Q_{T_{E}}=\left\{q_{0}, q_{1}, \ldots, q_{M}\right\}-$ внутренний алфавит машины $T_{E}$. По программе $P_{T_{E}}$ машины Тьюринга $T_{E}$ построим конечно определенную полугруппу $\Pi_{E}$, заданную множеством образующих элементов

$$
\left\{s_{0}, s_{1}, \ldots, s_{N}, q_{0}, q_{1}, \ldots, q_{M}, h, q\right\}
$$

и некоторым множеством определяющих соотношений $\mathscr{R}\left(T_{E}\right)$. Для этого мы используем ту же конструкцию, которая была использована при доказательстве теоремы 2.1.

I. Для каждой команды машины Тьюринга $T_{E}$ вида

$$
q_{i} s_{j} \mid q_{r} s_{t}
$$

включаем в множество определяюших соотношений $\mathscr{R}\left(T_{E}\right)$ соотношение $q_{i} s_{j}=q_{r} s_{t}$.

II. Для каждой команды вида

$$
q_{i} s_{j} \mid q_{r} L
$$

включаем в множество определяюших соотношений $\mathscr{R}\left(T_{E}\right)$ все соотношения вида $s_{t} q_{i} s_{j}=q_{r} s_{t} s_{j}$, где $t=0, \ldots, N$, и еше одно соотношение $h q_{i} s_{j}=h q_{r} s_{0} s_{j}$.

III. Для каждой команды $P_{T_{E}}(i, j)$ вида

$$
q_{i} s_{j} \mid q_{r} R
$$

и для каждого числа $t=0, \ldots, N$ включаем в множество определяющих соотношений $\mathscr{R}\left(T_{E}\right)$ соотношение $q_{i} s_{t} s_{j}=s_{t} q_{r} s_{j}$ и еше одно соотношение $q_{i} s_{j} h=s_{j} q_{r} s_{0} h$.

IV. Для каждого $j=0, \ldots, N$ включаем в множество $\mathscr{R}\left(T_{E}\right)$ два соотношения

$$
q_{0} s_{j}=q_{0}, \quad s_{j} q_{0}=q_{0} .
$$

Наконец, включим в $\mathscr{R}\left(T_{E}\right)$ еше и соотношение $h q_{0} h=q$.

Легко видеть, что если $W=q$ в $\Pi_{E}$ и $W$ отлично от $q$, то $W=h X_{1} q_{j} X_{2} h$, где $X_{1}$, $X_{2}-$ слова в алфавите $\left\{s_{0}, s_{1}, \ldots, s_{N}\right\}$.

Как было установлено ранее при доказательстве теоремы Маркова-Поста, верна следующая лемма.

ЛЕмма 9.1. Для полугруппь $\Pi_{\text {Е }}$ и любого слова $W$ в алфавите (6) выполнено следующее условие:

$$
W \in E \quad \Longleftrightarrow \quad h q_{1} W h=q \quad \text { в } \Pi_{E} .
$$

По полугруппе П ${ }_{E}$, используя конструкцию, описанную вьше при доказательстве теоремы 6.5, построим группу $G_{E}$.

Для удобства обозначим через

$$
\left\{a_{1}, \ldots, a_{n}\right\}
$$

все образуюшие полугруппы $\Pi_{E}$. Пусть $E_{1}=F_{1}, \ldots, E_{m}=F_{m}$ - все определяюшие соотношения $\Pi_{E}$, записанные в этом алфавите, т.е.

$$
\Pi_{E}=\left\langle a_{1}, a_{2}, \ldots, a_{n} \mid E_{1}=F_{1}, \ldots, E_{m}=F_{m}\right\rangle .
$$


Группу $G_{E}$ зададим образуюшими элементами

$$
a_{1}, a_{2}, \ldots, a_{n}, l, r, c, t, k
$$

и определяюшими соотношениями:

(i) $a_{j} l=l^{m+1} a_{j}(1 \leqslant j \leqslant n), r a_{j}=a_{j} r^{m+1}(1 \leqslant j \leqslant n)$,

(ii) $c a_{j}=a_{j} c(1 \leqslant j \leqslant n),\left(l^{i} E_{i} r^{i}\right) c=c\left(l^{i} F_{i} r^{i}\right)(1 \leqslant i \leqslant m)$,

(iii) $c t=t c, l t=t l$,

(iv) $c k=k c, r k=k r,\left(q^{-1} t q\right) k=k\left(q^{-1} t q\right)$.

Как и выше, будем использовать следуюшие обозначения:

$G_{1}$ - группа, заданная образуюшими $a_{1}, a_{2}, \ldots, a_{n}, l, r, c, t$ и определяющими соотношениями (i)-(iii),

$G_{2}$ - группа, заданная образуюшими $a_{1}, a_{2}, \ldots, a_{n}, l, r, c$ и определяюшими соотношениями (i) и (ii),

$G_{3}$ - группа, заданная образующими $a_{1}, a_{2}, \ldots, a_{n}, l, r$ и определяющими соотношениями (i),

а через $G_{0}$ обозначаем свободную группу, порожденную элементами $l, r$.

Напомним, что буква $k$ в группе $G_{E}$ есть правильная проходная буква и группа $G_{1}$ есть базис $G_{E}$ по этой проходной букве.

В группе $G_{1}$ правильной проходной буквой является буква $t$, а группа $G_{2}$ - базис $G_{1}$ по ней.

В группе $G_{2}$ буква $c$ - правильная проходная буква, а базисом при этом будет группа $G_{3}$.

Буквы $a_{1}, \ldots, a_{n}$ образуют в группе $G_{3}$ правильную систему проходных букв, а базисом в этом случае является свободная группа $G_{0}$.

Докажем несколько вспомогательных лемм.

Как и вьше, через $L$ и $R$ (с индексами или без них) будем обозначать слова вида $\varphi(l, c)$ и $\varphi(r, c)$, соответственно. Так как конструкция нашей групшы $G_{E}$ по полугруппе $\Pi_{E}$ совпадает с конструкцией группы $G$ по полугруппе П, приведенной в разделе 6 , то для нее верны доказанные ранее леммы $6.3,6.4$ и 6.5 с дополнительной информацией о связи с множеством $E$, которая отмечена в лемме 9.1 .

ЛЕмма 9.2. Элементы $\left\{a_{1}, \ldots, a_{n}, k\right\}$ являются в $G_{E}$ свободными образующими порожденной ими подгруппь. То же самое верно для множества $\left\{a_{1}, \ldots, a_{n}, t\right\}$.

ДокаЗАтельство. Допустим, что в группе $G_{E}$ вьполняется нетривиальное равенство $\varphi\left(a_{1}, \ldots, a_{n}, k\right)=1$. Рассмотрим кратчайшее из таких равенств. По лемме Бриттона слово $\varphi\left(a_{1}, \ldots, a_{n}, k\right)$ имеет вид $Z_{1} k^{\delta} T k^{-\delta} Z_{2}$, где $T \mp \psi\left(a_{1}, \ldots, a_{n}\right)$ и $\psi\left(a_{1}, \ldots, a_{n}\right)=\varphi_{1}\left(c, r, q^{-1} t q\right)$ в групше $G_{1}$.

Считаем, что слово $\varphi_{1}\left(c, r, q^{-1} t q\right)$ кратчайшее. Тогда, как было показано в начале доказательства леммы 6.5 , буквы $t^{ \pm 1}$ не должны входить в слово $\varphi_{1}\left(c, r, q^{-1} t q\right)$, т.е. $\varphi_{1}\left(c, r, q^{-1} t q\right)$ есть слово вида $R(c, r)$. Если бы в него входила буква $c$, то по лемме Бриттона мы получили бы равенство вида

$$
r^{i}=W\left(a_{1}, \ldots, a_{n}, l E_{1} r, l^{2} E_{2} r^{2}, \ldots, l^{m} E_{m} r^{m}\right),
$$


где $E_{1}, \ldots, E_{m}$ - непустые слова. Но это невозможно в силу леммы 6.2 . Следовательно, кратчайшее слово $\varphi_{1}\left(c, r, q^{-1} t q\right)$ должно быть степенью $r$. Тогда

$$
\psi\left(a_{1}, \ldots, a_{n}\right) \equiv 1
$$

в свободной группе $G_{0}$, т.е. слово $\varphi\left(a_{1}, \ldots, a_{n}, k\right)$ сократимо в свободной группе, что противоречит условию его выбора. Для элементов $\left\{a_{1}, \ldots, a_{n}, t\right\}$ доказательство аналогично.

Лемма 9.3. Если $X_{1}, X_{2}, X_{3}$ и $X_{4}$ - несократимые слова в алфавите $\left\{a_{1}, \ldots, a_{n}\right\}$ и в әруппе $G_{E}$ выполняется равенство

$$
\left(X_{1}^{-1} t X_{2}\right) k=k\left(X_{3}^{-1} t X_{4}\right)
$$

$m o X_{1}=X_{2}=X_{3}=X_{4}$.

ДокАЗАТЕЛЬСтво. Пусть $\left(X_{1}^{-1} t X_{2}\right) k=k\left(X_{3}^{-1} t X_{4}\right)$. Тогда, добавив соотношение $k=1$, получим, что в группе $G_{1}$, а значит, и в $G_{E}$ выполняется равенство $X_{1}^{-1} t X_{2}=X_{3}^{-1} t X_{4}$, откуда в силу леммы 9.2 получаем $X_{1} \mp X_{3}$ и $X_{2} \mp X_{4}$. Добавив соотношение $t=1$, получим, что в группе $G_{E}$ выполняется равенство $X_{1}^{-1} X_{2} k=k X_{3}^{-1} X_{4}$, откуда в силу леммы 9.2 получаем $X_{1}=X_{2}$ и $X_{3}=X_{4}$.

ЛЕмма 9.4. Пусть $H$ - подгруппа группь $G_{E}$, порожсденная әлементами $\left\{a_{1}, \ldots, a_{n}, t, k\right\}$. Тогда $H$ задается следующей системой определяющих соотношений:

$$
\left(X^{-1} t X\right) k=k\left(X^{-1} t X\right)
$$

где X пробегает множество всех слов, равньх $q$ в полугруппе $\Pi_{E}$.

ДокАЗАТЕльство. Обозначим через $H_{1}$ группу с образующими $\left\{a_{1}, \ldots, a_{n}, t, k\right\}$ и всеми соотношениями вида (7). Докажем, что группы $H$ и $H_{1}$ изоморфны.

По лемме 6.4 все соотношения групшы $H_{1}$ вьполнены в группе $H$.

Допустим, что $\varphi\left(a_{1}, \ldots, a_{n}, t, k\right)=1$ в $G_{E}$, но $\varphi\left(a_{1}, \ldots, a_{n}, t, k\right) \neq 1$ в группе $H_{1}$. Рассмотрим соотношение такого вида, содержашее минимальное число вхождений буквы $k$. В силу леммы 9.2 и леммы Бриттона

$$
\varphi\left(a_{1}, \ldots, a_{n}, t, k\right)=Z_{1} k^{\delta} T k^{-\delta} Z_{2},
$$

где слово $T$ не содержит вхождений буквы $k$ и коммутирует с ним, т.е. $k T k^{-1} T^{-1}=$ 1 в группе $G_{E}$. Кроме того, $k T k^{-1} T^{-1} \neq 1$ в группе $H_{1}$, так как иначе вместо $\varphi\left(a_{1}, \ldots, a_{n}, t, k\right)$ мы могли взять слово $Z_{1} t Z_{2}$, содержашее меншшее число вхождений $k$. Можно считать, что слово $T$ содержит минимальное число вхождений буквы $t$ при этих двух условиях.

В силу леммы 9.2 в слово $k T k^{-1} T^{-1}$ должно входить $t^{\delta}$. С другой стороны, так как $t-$ правильная проходная буква в группе $G_{E}$, то по лемме Бриттона

$$
k T k^{-1} T^{-1}=k Y t^{\delta} F t^{-\delta} Y_{1},
$$


где $F$ не содержит $t$ и коммутирует с $t$ в группе $G_{E}$. Согласно лемме $9.2 F$ содержит единственное вхождение буквы $k^{-1}$ в слово (9), т.е. слово $T$ имеет вид $Y t^{\delta} X$, а $F$ имеет вид $X k^{-1} X^{-1}$. Из соотношения

$$
t\left(X k X^{-1}\right)=\left(X k X^{-1}\right) t
$$

группы $G_{E}$ в силу леммы 6.5 следует $X=q$ в полугруппе $\Pi_{E}$, т.е. (10) выполнено и в группе $H_{1}$. Теперь, используя равенство (10) в группе $H_{1}$, мы можем уменьшить число вхождений буквы $t$ в слово $T$ при условии сохранения как равенства $k T k^{-1} T^{-1}=1$ в группе $G_{E}$, так и неравенства $k T k^{-1} T^{-1} \neq 1$ в группе $H_{1}$. Полученное противоречие завершает доказательство леммы 9.4.

Лемма 9.5. Подгруппа $H_{2}$ группь $G_{E}$, порожденная образующими

$$
\left\{k_{0}, t_{0}, b_{1}, b_{2}, \ldots, b_{p}\right\} \text {, }
$$

где $k_{0} \rightleftharpoons h k h^{-1}, t_{0} \rightleftharpoons q_{1}^{-1} h^{-1} t h q_{1}$, задается системой определяюших соотношений

$$
k_{0}^{-1}\left(W^{-1} t_{0} W\right) k_{0}=W^{-1} t_{0} W
$$

где $W$ пробегает все слова из $E$.

ДокАЗАТЕЛЬСТво. Обозначим через $H_{3}$ группу, заданную образуюшими $(11)$ и всеми определяюшими соотношениями вида (12).

Если $W \in E$, то $h q_{1} W h=q$ в полугруппе $\Pi_{E}$. Тогда по лемме 6.4 в группе $G_{E}$ вьполнено равенство

$$
\left(h^{-1} W^{-1} q_{1}^{-1} h^{-1} t h q_{1} W h\right) k=k\left(h^{-1} W^{-1} q_{1}^{-1} h^{-1} t h q_{1} W h\right),
$$

т.е. равенство $k_{0}^{-1}\left(W^{-1} t_{0} W\right) k_{0}=W^{-1} t_{0} W$. Таким образом, все соотношения (12) выполнены в группе $\mathrm{H}_{2}$.

Ограничившись рассмотрением слов вида $\varphi\left(b_{1}, \ldots, b_{p}, t_{0}, k_{0}\right)$, равных 1 в $G_{E}$, и повторив рассуждения, приведенные при доказательстве предыдущей леммы, мы получим, что в $H_{3}$ вьполнены все соотношения подгруппы $H_{2}$, т.е. $H_{2} \simeq H_{3}$. При этом мы воспользуемся тем, что в данном случае в представлении (8) слово $T$ имеет вид $h^{-1} T_{1} h$, а в представлении (9) слово $F$ имеет вид $h q_{1} F_{1} q_{1}^{-1} h^{-1}$. При этом соотношение (10) будет иметь вид

$$
t\left(h q_{1} X h k h^{-1} X^{-1} q_{1}^{-1} h^{-1}\right)=\left(h q_{1} X h k h^{-1} X^{-1} q_{1}^{-1} h^{-1}\right) t .
$$

Отсюда в силу леммы 6.5 получим $h q_{1} X h=q$ в полугруппе $\Pi_{E}$, т.е. $X \in E$. При этом полученное соотношение (13) имеет вид (12) и оно выполнено в $H_{3}$.

Для произвольного слова $W\left(b_{1}, \ldots, b_{p}\right)$ в алфавите $\left\{b_{1}, \ldots, b_{p}\right\}$ через $W_{u}$ будем обозначать результат замены в $W\left(b_{1}, \ldots, b_{p}\right)$ каждой буквы $b_{i}(i=1, \ldots, p)$ на новую букву $u_{i}$, а через $W_{c}$ - результат подстановки в $W\left(b_{1}, \ldots, b_{p}\right)$ вместо каждой буквы $b_{i}$ $(i=1, \ldots, p)$ новой буквы $c_{i}$. При этом само слово $W$ иногда будем обозначать чеpeз $W_{b}$. 
ОПРЕДЕЛЕНИЕ. Для произвольной группы $G$ через

$$
\left\langle\left\langle G ; c_{1}, \ldots, c_{n} \mid C_{1}=D_{1}, \ldots, C_{m}=D_{m}\right\rangle\right\rangle
$$

будем обозначать группу, задание которой получается из задания $G$ добавлением новых образуюших $c_{1}, \ldots, c_{n}$ и новых определяюших соотношений $C_{1}=D_{1}, \ldots$, $C_{m}=D_{m}$.

В дополнение к построенным нами группам $G_{E}, G_{1}, G_{2}$ и $G_{3}$ рассмотрим следуюшие группы. Положим

$$
\begin{aligned}
R & \rightleftharpoons\left\langle\left\langle u_{1}, u_{2}, \ldots, u_{p} \mid\left\{W_{u}=1 \mid W \in E\right\}\right\rangle\right\rangle ; \\
G_{4} & \rightleftharpoons G_{E} * R=\left\langle\left\langle G_{E} ; u_{1}, u_{2}, \ldots, u_{p} \mid\left\{W_{u}=1 \mid W \in E\right\}\right\rangle\right\rangle ; \\
G_{5} & \rightleftharpoons\left\langle\left\langle G_{4} ; c_{1}, c_{2}, \ldots, c_{p} \mid\left\{c_{i} b_{j}=b_{j} c_{i}, c_{i} u_{j}=u_{j} c_{i}, c_{i} k_{0} u_{i}^{-1}=k_{0} c_{i} \mid i, j=1, \ldots, p\right\}\right\rangle\right\rangle ; \\
G_{6} & \rightleftharpoons\left\langle\left\langle G_{5} ; d \mid d k_{0}=k_{0} d,\left\{d b_{i}=b_{i} c_{i} d \mid i=1, \ldots, p\right\}\right\rangle\right\rangle ; \\
G_{7} & \rightleftharpoons\left\langle\left\langle G_{6} ; g \mid t_{0} g=g t_{0} d, k_{0} g=g k_{0},\left\{b_{i} g=g b_{i} \mid i=1, \ldots, p\right\}\right\rangle\right\rangle .
\end{aligned}
$$

I) В силу леммы 9.2 элементы $b_{1}, \ldots, b_{p}, k_{0}$ являются свободными образующими порождаемой ими подгруппы в группе $G_{E}$, а значит, и в группе $G_{4}$. Поэтому элементы $b_{1}, \ldots, b_{p}, u_{1}, \ldots, u_{p}, k_{0}$ - система свободных образующих для подгруппы, порожденной ими в группе $G_{4}$. Аналогично, $b_{1}, \ldots, b_{p}, u_{1}, \ldots, u_{p}, k_{0} u_{i}^{-1}$ при каждом фиксированном $i(i=1, \ldots, p)$ есть система свободных образуюших для подгруппы, порожденной ими в группе $G_{4}$. Отсюда следует, что $c_{1}, \ldots, c_{p}$ - правильная система проходных букв в $G_{5}$.

II) Очевидно, $b_{1} c_{1}, \ldots, b_{p} c_{p}, k_{0}$ являются свободными образуюшими подгруппы, порожденной ими в группе $G_{5}$. Поэтому $d$ - правильная проходная буква в $G_{6}$.

III) Докажем следуюшее утверждение:

$$
\varphi\left(t_{0}, k_{0}, b_{1}, \ldots, b_{p}\right)=1 \text { в } G_{6} \Longleftrightarrow \varphi\left(t_{0} d, k_{0}, b_{1}, \ldots, b_{p}\right)=1 \text { в } G_{6} .
$$

Если в группе $G_{6}$ выполняется равенство $\varphi\left(t_{0} d, k_{0}, b_{1}, \ldots, b_{p}\right)=1$, то, добавив соотношение $d=1$, получим $\varphi\left(t_{0}, k_{0}, b_{1}, \ldots, b_{p}\right)=1$.

Пусть в группе $G_{6}$ вьполняется равенство $\varphi\left(t_{0}, k_{0}, b_{1}, \ldots, b_{p}\right)=1$. В силу леммы 9.5 оно выводимо из соотношений вида $(12)$, где $W \in E$. Заметим, что в группе $G_{6}$ вьполняются равенства

$$
d W_{b}=W_{b} W_{c} d
$$

а в групше $G_{5}-$ равенства

$$
W_{c} k_{0}=k_{0} W_{c} W_{u} .
$$

Кроме того, если $W_{b} \in E$, то $W_{u}=1$ в группе $G_{4}$. Поэтому все эти равенства выполняются и в $G_{6}$. Используя эти равенства, из соотношений $(12)$ легко выведем соотношения

$$
\left(W_{b}^{-1} t_{0} d W_{b}\right) k_{0}=k_{0}\left(W_{b}^{-1} t_{0} d W_{b}\right),
$$

а из них - равенство $\varphi\left(t_{0} d, k_{0}, b_{1}, \ldots, b_{p}\right)=1$. 
Из доказанной эквивалентности (14) следует, что $g$ - правильная проходная буква в групше $G_{7}$.

IV) Из пунктов I), II) и III) следует, что группа $R$ является подгруппой группы $G_{7}$.

Для завершения доказательства теоремы Хигмана нам остается доказать, что $G_{7}$ конечно определенная группа. Обозначим через $G_{7}^{\prime}$ группу, задание которой получается из задания групшы $G_{7}$ удалением всех определяюших соотношений $W_{u}=1$ исходной группы $R$.

В силу (10) для произвольного слова $W_{b} \in E$ в $G_{7}^{\prime}$ вьполняется равенство

$$
k_{0}^{-1}\left(W_{b}^{-1} t_{0} W_{b}\right) k_{0}=W_{b}^{-1} t_{0} W_{b} .
$$

Умножив обе части этого равенства справа на $g$ и проташив $g$ влево в обеих частях равенства, получим после сокрашения на $g$ равенство

$$
k_{0}^{-1}\left(W_{b}^{-1} t_{0} d W_{b}\right) k_{0}=W_{b}^{-1} t_{0} d W_{b} .
$$

Далее, используя соотношения (15), (16) и $(17)$, получим в $G_{7}^{\prime}$ равенства

$$
\begin{aligned}
k_{0}^{-1} W_{b}^{-1} t_{0} W_{b} W_{c} d k_{0} & =W_{b}^{-1} t_{0} W_{b} W_{c} d, \\
k_{0}^{-1} W_{b}^{-1} t_{0} W_{b} k_{0} W_{c} W_{u} d & =W_{b}^{-1} t_{0} W_{b} W_{c} d, \\
W_{b}^{-1} t_{0} W_{b} W_{c} W_{u} d & =W_{b}^{-1} t_{0} W_{b} W_{c} d .
\end{aligned}
$$

Следовательно, $W_{u}=1$ в группе $G_{7}^{\prime}$. Поэтому группы $G_{7}$ и $G_{7}^{\prime}$ изоморфны. Это завершает доказательство теоремы Хигмана.

СлЕДСТвИЕ 9.1.1. Существует конечно определенная группа, универсальная для всех конечно определенных групп, т.е. такая конечно определенная группа $F$, что для любой конечно определенной группы $G$ в $F$ есть подгруппа, изоморфная $G$.

ДОкАЗАТЕЛЬСТво. Пусть задан некоторый бесконечньй алфавит

$$
a_{1}, a_{2}, \ldots, a_{i}, a_{i+1}, \ldots
$$

Очевидно, любая конечно определенная группа $G$ с $k$ образуюшими может быть задана в алфавите $\left\{a_{i}, a_{i+1}, \ldots, a_{i+k-1}\right\}$. С другой стороны, имеется только счетное число различных заданий конечно определенных групп с образуюшими из (18), и мы имеем простой алгоритм для перечисления всех таких заданий. Пусть

$$
G_{1}, G_{2}, G_{3}, \ldots, G_{j}, G_{j+1}, \ldots
$$

есть рекурсивно перечислимая последовательность заданий конечно определенных групп в алфавите (18), в которой с точностью до изоморфизма представлены все конечно определенные группы. Можно считать, что алфавиты групп (19) не пересекаются и для каждого $j$ если в группах $G_{1}, \ldots, G_{j}$ использованы буквы из отрезка $a_{1}, a_{2}, \ldots, a_{i}$, то алфавит группы $G_{j+1}$ есть начальный отрезок последовательности

$$
a_{i+1}, a_{i+2}, \ldots, a_{i+r}, \ldots
$$


Объединив все определяющие соотношения групп последовательности (19), мы получим группу $G$, заданную рекурсивно перечислимым множеством определяющих соотношений в алфавите (18) и универсальную для всех конечно определенных групп. Нам остается сослаться на лемму 8.2 .

М. К. Валиевым в работе [107] был построен явньй пример универсальной конечно определенной групшы с 14 образуюшими и 42 определяюшими соотношениями.

Из теоремы Хигмана выводится следуюшая алгебраическая характеризация групш с разрешимой проблемой распознавания равенства слов, которая была получена в работе [23].

ТЕОРема 9.2. В конечно порожденной группе проблема равенства слов разрешима тогда и только тогда, когда эта группа вложима в простую подгруппу некоторой конечно определенной группь.

ДокАЗАТЕЛЬство. Пусть конечно порожденная группа $G$ с образуюшими $u_{1}, \ldots, u_{n}$ есть подгруппа простой группы $S$, которая в свою очередь есть подгруппа конечно определенной группы $H$ с образующими $b_{1}, \ldots, b_{k}$ и определяющими соотношениями $R_{1}=1, \ldots, R_{m}=1$. Докажем разрешимость проблемы равенства слов в $G$.

Можно считать, что группа $G$ неединичная. Пусть $W\left(u_{1}, \ldots, u_{n}\right)$ - произвольньй ее элемент. Обозначим через $H_{W}$ группу с образующими $b_{1}, \ldots, b_{k}$ и определяюшими соотношениями $R_{1}=1, \ldots, R_{m}=1$ и $W_{1}\left(b_{1}, \ldots, b_{k}\right)=1$, где $W_{1}$ - результат подстановки $u_{i}=u_{i}\left(b_{1}, \ldots, b_{k}\right.$, реализующей вложение $G$ в группу $H$.

Одновременно выполняем два алгоритма $-\mathfrak{A}$ и $\mathfrak{B}$.

Пусть алгоритм $\mathfrak{A}$ перечисляет все слова в образуюших $b_{1}, \ldots, b_{k}$, равные 1 в группе $H$, а алгоритм $\mathfrak{B}$ перечисляет все слова в образуюших $b_{1}, \ldots, b_{k}$, равные 1 в группе $H_{W}$.

Если $W_{1}\left(b_{1}, \ldots, b_{k}\right)=1$ в группе $G$, то слово $W_{1}\left(b_{1}, \ldots, b_{k}\right)$ будет найдено алгоритмом $\mathfrak{A}$. Если же $W_{1}\left(b_{1}, \ldots, b_{k}\right) \neq 1$ в $G$, то в силу простоты подгруппы $S$ в процессе работы алгоритма $\mathfrak{B}$ будет установлено, что все образуюшие $u_{1}, \ldots, u_{n}$ групшы $G$ равны 1 в группе $H_{W}$. Так как группа $G$ неединичная, то в результате совместной работы этих двух алгоритмов будет доказано равенство $W_{1}\left(b_{1}, \ldots, b_{k}\right)=1$ или его отрицание в $G$.

Пусть конечно порожденная группа $G$ имеет разрешимую проблему равенства. Рассмотрим рекурсивно перечислимое множество всех упорядоченных пар $\left\langle A_{i}, B_{i}\right\rangle$ неединичных элементов группы $G$. Пусть $\alpha$ - новая буква. В свободном произведении $G *\langle\langle\alpha\rangle\rangle$ элементы $A_{i} \alpha A_{i} \alpha^{-1}$ и $B_{i} \alpha A_{i}^{-1} \alpha^{-1}$ имеют бесконечньй порядок. Поэтому группа

(20) $G^{\prime} \rightleftharpoons\left\langle\left\langle G *\langle\langle\alpha\rangle\rangle ; t_{i}(i=1,2, \ldots) \mid t_{i} A_{i} \alpha A_{i} \alpha^{-1} t_{i}^{-1}=B_{i} \alpha A_{i}^{-1} \alpha^{-1}(i=1,2, \ldots)\right\rangle\right\rangle$

есть HNN-расширение группы $G *\langle\langle\alpha\rangle\rangle$.

Так как в группе $G$ разрешима проблема равенства, а в группе $G *\langle\langle\alpha\rangle\rangle$ разрешимы проблемы вхождения в циклические подгруппы, порожденные элементами $A_{i} \alpha A_{i} \alpha^{-1}$ и $B_{i} \alpha A_{i}^{-1} \alpha^{-1}$, то по теореме 6.3 в групше $G^{\prime}$ разрешима проблема равенства. Группа $G^{\prime}$ рекурсивно определена, и для любых двух неединичных элементов $A_{i}$ и $B_{i}$ группы $G$ в группе $G^{\prime}$ найдутся такие элементы $U_{1}, U_{2}$ и $U_{3}$, что выполняется равенство

$$
B_{i}=\left(U_{1} A_{i} U_{1}^{-1}\right)\left(U_{2} A_{i} U_{2}^{-1}\right)\left(U_{3} A_{i} U_{3}^{-1}\right) .
$$


Положим $G_{0} \rightleftharpoons G$ и $G_{j+1} \rightleftharpoons\left(G_{j}\right)^{\prime}$ при $j=1,2, \ldots$ Группа

$$
G^{*} \rightleftharpoons \bigcup_{j=0}^{\infty} G_{j}
$$

является рекурсивно определенной, имеет разрешимую проблему равенства и для любых двух ее неединичных элементов $A$ и $B$ найдутся такие элементы $U_{1}, U_{2}$ и $U_{3}$ группы $G^{*}$, что вьполнено равенство

$$
B=\left(U_{1} A U_{1}^{-1}\right)\left(U_{2} A U_{2}^{-1}\right)\left(U_{3} A U_{3}^{-1}\right) .
$$

В частности, это означает, что группа $G^{*}$ является простой. В силу леммы 8.2 эта группа вложима в 2-порожденную группу, которая будет рекурсивно определенной, а значит, по теореме Хигмана она вложима в конечно определенную группу.

Пока остается открытым вопрос: можно ли произвольную конечно порожденную группу с разрешимой проблемой равенства вложить в конечно определенную простую группу?

Следуюшая теорема доказана Ч. Миллером III [65].

Теорема 9.3. Существует такая конечно определенная группа, все нетривиальные факторгруппы которой имеют неразрешимую проблему равенства слов.

ДокАЗАТЕЛЬСтво. Напомним, что непересекающиеся множества $K$ и $T$ натуральных чисел называются рекурсивно неотделимыми, если не сушествует такого рекурсивного расширения множества $K$, которое не пересекалось бы с $T$. Пусть $K, T$ - пара рекурсивно перечислимых множеств натуральных чисел, которые рекурсивно неотделимы. Можно считать, что $1 \in K, 2 \in T$.

По лемме 8.2 и теореме Хигмана группа $G_{0}$, заданная порождаюшими $a_{1}, a_{2}, \ldots$, $a_{i}, \ldots$ и определяюшими соотношениями $a_{i}=a_{1}$ для всех $i \in K$ и $a_{i}=a_{2}$ для всех $i \in T$, вложима в некоторую конечно определенную групту $G$.

Так же, как это было сделано в лемме 8.1 , по неединичному элементу $w \rightleftharpoons a_{1} a_{2}^{-1}$ группы $G$ построим конечно определенную группу $G_{w}$, являюшуюся расширением группы $G$ и такую, что нормальное замыкание элемента $w$ совпадает со всей группой $G_{w}$. Сохраним обозначения $a_{i}$ для их образов в группах $G$ и $G_{w}$.

Рассмотрим произвольную неединичную факторгруппу $H$ группы $G_{w}$. Очевидно, $a_{1}^{-1} a_{2} \neq 1$ в $H$. Поэтому множество

$$
\left\{n \mid a_{1}=a_{n} \text { в группе } H\right\}
$$

содержит $K$ и не пересекается с $T$. В силу рекурсивной неотделимости множеств $K$ и $T$ множество (21) нерекурсивно, т.е. проблема распознавания равенства в $H$ неразрешима для класса слов $\left\{a_{1}^{-1} a_{n} \mid n=1,2, \ldots\right\}$.

Приведем еще одну интересную характеризацию конечно порожденњых групп с разрешимой проблемой равенства слов. Она формулируется в терминах алгебраически замкнутых групп. Группа $G$ называется алгебраически замкнутой, если любая система уравнений и неравенств с переменными и коэффициентами из $G$, имеюшая решение в некотором расширении $G$, имеет решение и в самой группе $G$. 
Алгебраически замкнутые группы не являются конечно порожденньми группами, так как в них любое конечное множество элементов имеет нетривиальньй централизатор, в то время как центр всей групшы тривиален (последнее следует из разрешимости неравенства $a x \neq x a)$.

Легко доказать, что любая алгебраически замкнутая группа $G$ является простой. В самом деле, как было показано вьше, если $A$ и $B$ - неединичные элементы групшы $G$, то уравнение

$$
A=\left(U_{1} B U_{1}^{-1}\right)\left(U_{2} B U_{2}^{-1}\right)\left(U_{3} B U_{3}^{-1}\right)
$$

имеет решение в некотором расширении (20) группы $G$. Тогда оно разрешимо и в $G$. Это означает, что любой элемент $A$ принадлежит нормальному замыканию любого неединичного элемента $B$.

Б. Нейман [67] доказал, что каждая конечно порожденная группа с разрешимой проблемой равенства слов вложима в любую алгебраически замкнутую группу. В работе [49] А. Макинтайр получил обращение этой теоремы, доказав, что если конечно порожденная группа вложима в любую алгебраически замкнутую группу, то в ней разрешима проблема равенства слов. Он также заметил, что из теоремы 9.3 следует, что каждая алгебраически замкнутая группа имеет некоторую конечно порожденную подгруппу с неразрешимой проблемой равенства слов.

\section{СПИСОК ЛИТЕРАТУРЫ}

[1] Aanderaa S. A proof of Higman's embedding theorem using Britton extentions of groups // Stud. Logic Found. Math. 1973. V. 71. P. 1-18.

[2] Адян С.И. Алгоритмическая неразрешимость проблем распознавания некоторых свойств групп // Докл. АН СССР. 1955. Т. 103. №4. С. 533-535.

[3] Адян С.И. Неразрешимость некоторых алгоритмических проблем теории групп // Труды ММO. 1957. Т. 6. С. 231-298.

[4] Адян С.И. Роль закона сокращения при задании полугрупп с сокращением посредством определяющих соотношений // Докл. АН СССР. 1957. Т. 113. №6. С. 1191-1194.

[5] Адян С.И. Конечно-определенные группы и алгоритмы // Докл. АН СССР. 1957. T. 117. №1. С. $9-12$.

[6] Адян С.И. Об алгоритмических проблемах в эффективно-полных классах групп // Докл. АН СССР. 1958. Т. 123. №1. С. 13-16.

[7] Адян С.И.О вложимости полугрупп в группы // Докл. АН СССР. 1960. Т. 133. № 2. C. $255-257$.

[8] Адян С.И.К проблеме тождества в ассоциативных исчислениях специального вида // Докл. АН СССР. 1960. Т. 135. № 6. С. 1297-1300.

[9] Адян С.И. Тождества в специальных полугруппах // Докл. АН СССР. 1962. Т. 143. № 3. C. 499-502.

[10] Адян С. И. Определяющие соотношения и алгоритмические проблемы для групп и полугрупп. М.: Наука, 1966. (Труды МИАН. Т. 85.)

[11] Адян С.И. Бесконечные неприводимые системы групповых тождеств // Докл. АН CCCP. 1970. T. 190. № 3. С. 499-501.

[12] Адян С.И. Бесконечные неприводимые системы групповых тождеств // Изв. АН СССР. Сер. матем. 1970. Т. 34. № 4. С. 715-734.

[13] Адян С. И. Проблема Бернсайда и тождества в группах. М.: Наука, 1975.

[14] Адян С.И. О преобразованиях слов в полугруппе, заданной системой определяющих соотношений // Алгебра и логика. 1976. Т. 15. №6. С. 611-621.

[15] Адян С.И. К проблеме делимости для моноидов, заданных одним определяющим соотношением // Матем. заметки. 1994. Т. 55. №1. С. 3-9.

[16] Адян С.И. Нормальные подгруппы свободных периодических групп // Изв. АН СССР. Сер. матем. 1981. Т. 45. № 5. С. 931-947. 
[17] Адян С.И., Оганесян Г.У. К проблемам равенства и делимости в полугруппах с одним определяющим соотношением // Изв. АН СССР. Сер. матем. 1978. Т. 42. № 2 . C. $219-225$.

[18] Адян С.И., Оганесян Г.У. О проблемах равенства и делимости в полугруппах с одним определяющим соотношением // Матем. заметки. 1987. Т. 41. № 3. С. 412-421.

[19] Безверхний В.Н. Решение проблемы вхождения для одного класса групп // Вопросы теории групп и полугрупп. № 2. Тула: ТГПИ им. Л.Н. Толстого, 1972. С. 3-86.

[20] Бокуть Л.А., Кукин Г.П. Неразрешимые алгоритмические проблемы для полугрупп, групп и колец // Итоги науки и техники. Алгебра. Топология. Геометрия. Т. 25. М.: ВИНИТИ, 1987. С. 3-66.

[21] Boone W.W. Certain simple unsolvable problems of group theory. V, VI // Proc. Konink. Nederl. Akad. Wetensch. Ser. A. 1957. V. 60. P. 22-27; 227-232.

[22] Boone W.W. The word problem // Ann. of Math. 1959. V. 70. № 2. P. 207-265.

[23] Boone W. W., Higman G. An algebraic characterization of the solvability of the word problem // J. Austral. Math. Soc. 1974. V. 18. №1. P. 41-53.

[24] Boone W. W., Rogers H. Jr. On a problem of J.H.C.Whitehead and a problem of Alonzo Church // Math. Scand. 1966. V. 19. № 2. P. 185-192.

[25] Борисов В. В.Простые примеры групп с неразрешимой проблемой тождества // Матем. заметки. 1969. Т. 6. № 5. С. 521-532.

[26] Britton J. L. The word problem for groups // Proc. London Math. Soc. (3). 1958. V. 8. P. 493-506.

[27] Britton J. L. The word problem // Ann. of Math. (2). 1963. V. 77. № 1. P. 16-32.

[28] Чеботарь А.А. Подгруппы групп с одним определяющим соотношением // Известия высших учебных заведений. Математика. 1978. № 8 (195). С. 109-118.

[29] Churh A. An unsolvable problem of elementary number theory // Amer. J. Math. 1936. V. 58. №2. P. 345-363.

[30] Churh A. A note on the Entscheidungsproblem // J. Symbolic Logic. 1936. V. 1. № 1. P. 40-41.

[31] Dehn M. Über unendliche diskontinuerliche Gruppen // Math. Ann. 1912. V. 71. P. 116-144.

[32] Епанчинцев В.И., Кукин Г.П. Проблема равенства в многообразии групп, содержащем $\mathfrak{N}_{2} \mathfrak{A} / /$ Алгебра и логика. 1979. Т. 18. №3. С. 259-285.

[33] Фридман А.А. О взаимоотношении между проблемой тождества и проблемой сопряженности в конечно определенных группах // Труды ММО. 1960. Т. 9. С. 329-356.

[34] Гольберг А.И. О невозможности усиления некоторых результатов Гриндлингера и Линдона // УМН. 1978. Т. 33. №6. С. 201-202.

[35] Greendlinger M. Dehn's algorithm for the word problem // Comm. Pure Appl. Math. 1960. V. 13. P. $67-83$.

[36] Greendlinger M. On Dehn's algorithms for the conjugacy and word problems with applications // Comm. Pure Appl. Math. 1960. V. 13. P. 641-677.

[37] Grunewald F., Segal D. Some general algorithms. I: Arithmetic groups; II: Nilpotent groups // Ann. of Math. (2). 1980. V. 112. № 3. P. 531-583; 585-617.

[38] Hall P. On the finiteness of certain soluble groups // Proc. London Math. Soc. 1959. V. 9. P. 592-622.

[39] Higman G. Subgroups of finitely presented groups // Proc. Roy. Soc. London. Ser. A. 1961. V. 262. № 1311. P. 455-475.

[40] Higman G., Neumann B.H., Neumann H. Embedding theorems for groups // J. London Math. Soc. 1949. V. 24. P. 247-254.

[41] Howie J., Pride S. The word problem for one-relator semigroups // Proc. Cambridge Philos. Soc. 1986. V. 99. № 1. P. 33-44.

[42] van Kampen E. R. On some lemmas in the theory of groups // Amer. J. Math. 1933. V. 55. P. 268-273.

[43] Харлампович О.Г. Конечно определенная разрешимая группа с неразрешимой проблемой равенства // Изв. АН СССР. Сер. матем. 1981. Т. 45. № 4. С. 852-873.

[44] Клейман Ю. Г. О тождествах в группах // Труды ММО. 1982. Т. 44. С. 62-108. 
[45] Курош А. Г. Теория групп. М.: Наука, 1967.

[46] Lyndon R. C. On Dehn's algorithm // Math. Ann. 1966. V. 166. P. 208-228.

[47] Линдон Р., Шупп П. Комбинаторная теория групп. М.: Мир, 1980.

[48] Лысенок И. Г. Бесконечные бернсайдовы группы четного периода // Изв. РАН. Сер. матем. 1996. T. 60. № 3. С. 3-224.

[49] Macintyre A. On algebraically closed groups // Ann. of Math. (2). 1972. V. 96. №1. P. 53-97.

[50] Magnus W. Das Identitäts problem für Gruppen mit einer definierenden Relation // Math. Ann. 1932. V. 106. P. 295-307. Рус. пер.: Проблема идентичности для групп с одним определяющим соотношением // УМН. 1940. Вып. 8. С. 365-376.

[51] Магнус В., Каррас А., Солитэр Д. Комбинаторная теория групп. М.: Наука, 1974.

[52] Маканин Г.С. К проблеме тождества в конечно-определенных полугруппах // Докл. AH CCCP. 1966. T. 171. № 2. C. 285-287.

[53] Маканин Г. С. К проблеме тождества в конечно-определенных группах и полугруппах // Дисс. ... канд. физ.-матем. наук. М: МИАН, 1966.

[54] Маканина Т. А. Проблема вхождения для группы кос $\mathfrak{B}_{n}$ при $n \geqslant 5$ // Матем. заметки. 1981. Т. 29. № 1. С. 31-33.

[55] Мальцев А.И. О гомоморфизмах на конечные группы // Уч. зап. Ивановск. гос. пед. ин-та 1958. Т. 18. № 5. С. 49-60.

[56] Мальцев А. И. Алгоритмы и рекурсивные функции. М.: Наука, 1965.

[57] Марков А.А. Невозможность некоторых алгорифмов в теории ассоциативных систем // Докл. АН СССР. 1947. Т. 55. № 7. С. 587-590.

[58] Марков А. А. Теория алгорифмов. М.: Наука, 1954. (Труды МИАН. Т. 42.)

[59] Марков А.А. Неразрешимость проблемы гомеоморфии // Докл. АН СССР. 1958. T. 121. № 2. C. $218-220$.

[60] Марков А. А. К проблеме представимости матриц // Z. Math. Logik Grundlag. Math. 1958. V. 4. № 2. P. 157-168.

[61] Матиясевич Ю.В.Простые примеры неразрешимых ассоциативных исчислений // Докл. АН СССР. 1967. Т. 173. № 6. С. 1264-1266.

[62] Матвеев С. В. Алгоритм распознавания трехмерной сферы (по А. Томпсон) // Матем. сб. 1995. Т. 186. № 5. С. 69-84.

[63] Михайлова К. А. Проблема вхождения для прямых произведений групп // Докл. АН CCCP. 1958. T. 119. C. 1103-1105.

[64] Михайлова К.А. Проблема вхождения для свободных произведений групп // Докл. AH CCCP. 1959. T. 127. C. 746-748.

[65] Miller C. F. III. Decision problems for groups-survey and reflections // Math. Sci. Res. Inst. Publ. 1992. V. 23. P. 1-59.

[66] Мурский В.Л. Изоморфная вложимость полугруппы с перечислимым множеством определяющих соотношений в конечно определенную полугруппу // Матем. заметки. 1967. T. 1. № 2. C. 217-224.

[67] Neumann B. H. The isomorphism problem for algebraically closed groups // Stud. Logic Found. Math. 1973. V. 71. P. 553-562.

[68] Newman B. B. Some results on one-relator groups // Bull. Amer. Math. Soc. 1968. V. 74. P. 568-571.

[69] Nielsen J. Om Regning med ikke-kommutative Faktoren og dens Anvendelse i Gruppeteorien // Mathematisk Tidsskrift B. 1921. P. 77-94.

[70] Новиков П. С. Об алгоритмической неразрешимости проблемы тождества // Докл. АН CCCP. 1952. T. 85. № 4. С. 709-712.

[71] Новиков П.С. Неразрешимость проблемы сопряженности в теории групп // Изв. АН СССР. Сер. матем. 1954. Т. 18. №6. С. 485-524.

[72] Новиков П. С. Об алгоритмической неразрешимости проблемы тождества слов в теории групп. М.: Наука, 1955. (Труды МИАН. Т. 44.)

[73] Новиков П. С., Адян С.И. Проблема тождества для полугрупп с односторонним сокращением // Z. Math. Logik Grundlag. Math. 1958. V. 4. № 1. P. 66-88. 
[74] Новиков П. С., Адян С. И. О бесконечных периодических группах. I; II; III // Изв. АН СССР. Сер. матем. 1968. Т. 32. №1. С. 212-244; № 2. С. 251-524; № 3. С. 709-731.

[75] Новиков П.С., Адян С.И. Определяющие соотношения и проблема тождества для свободных периодических групп нечетного порядка // Изв. АН СССР. Сер. матем. 1968. T. 32. № 4. C. 971-979.

[76] Новиков П. С., Адян С. И. О коммутативных подгруппах и проблеме сопряженности в свободных периодических группах нечетного порядка // Изв. АН СССР. Сер. матем. 1968. Т. 32. № 5. C. 1176-1190.

[77] Оганесян Г. У. О полугруппах с одним соотношением и полугруппах без циклов // Изв. АН СССР. Сер. матем. 1982. Т. 46. № 1. С. 88-94.

[78] Ольшанский А. Ю. Геометрия определяющих соотношений в группах. М.: Наука, 1989.

[79] Ольшанский А. Ю., Шмелькин А. Л. Бесконечные группы // Итоги науки и техники. Современные проблемы математики. Фундаментальные направления. Т. 37. М.: ВИНИТИ, 1989. С. 5-113.

[80] Осипова В.А. К проблеме тождества для конечно-определенных полугрупп // Докл. AH CCCP. 1968. Т. 178. № 5. С. 1017-1020.

[81] Осипова В.А.Алгоритмические проблемы в полугруппах с ограниченной мерой налегания определяющих слов // Дисс. . . . канд. физ.-матем. наук. М.: МИАН, 1971.

[82] Павлов Р. Д. К проблеме распознавания групповых свойств // Матем. заметки. 1971. Т. 10. № 2. С. 169-180.

[83] Post E. L. Finite combinatory processes-formulation 1 // J. Symbolic Logic. 1936. V. 1. № 3. P. 103-105.

[84] Post E. L. Recursive unsolvability of a problem of Thue // J. Symbolic Logic. 1947. V. 12. № 1. P. 1-11.

[85] Rabin M. O. Recursive unsolvability of group theoretic problems // Ann. of Math. 1958. V. 67. № 1. P. 172-194.

[86] Ремесленников В. Н. Пример группы, конечно-определенной в многообразии $\mathfrak{A}^{5}, \mathrm{c}$ неразрешимой проблемой равенства слов // Алгебра и логика. 1973. Т. 12. № 5. С. 577-602.

[87] Ремесленников В.Н., Романьков В.А. Теоретико-модельные и алгоритмические вопросы теории групп // Итоги науки и техники. Алгебра. Топология. Геометрия. Т. 21. М.: ВИНИТИ, 1983. С. 3-79.

[88] Романовский Н. С. О некоторых алгоритмических проблемах для разрешимых групп // Алгебра и логика. 1974. Т. 13. № 1. С. 26-34.

[89] Романовский Н. С. Свободные подгруппы в конечно определенных группах // Алгебра и логика. 1977. Т. 16. №1. С. 88-97.

[90] Sapir M. V. On the word problem in periodic group varieties // Internat. J. Algebra Comput. 1991. V. 1. №1. P. 115-126.

[91] Саркисян О.А. Некоторые соотношения между проблемами тождества и делимости в группах и полугруппах // Изв. АН СССР. Сер. матем. 1979. Т. 43. № 4. С. 909-921.

[92] Саркисян О.А.О проблемах равенства и делимости в группах и полугруппах без циклов // Изв. АН СССР. Сер. матем. 1981. Т. 45. №6. С. 1424-1440.

[93] Саркисян Р. А.Алгоритмические вопросы для линейных алгебраических групп I; II // Матем. сб. 1980. Т. 113. № 2. С. 179-216; № 3. С. 400-436.

[94] Scott D. A short recursively unsolvable problem (abstract) // J. Symbolic Logic. 1956. V. 21. № 1. P. 11-112.

[95] Scott E. A. A finitely presented simple group with unsolvable conjugacy problem // J. Algebra. 1984. V. 90. № 2. P. 333-353.

[96] Тайцлин М. А. О проблеме изоморфизма для коммутативных полугрупп // Матем. сб. 1974. Т. 93. № 1. С. 103-128.

[97] Тайцлин М. А. Проблема изоморфизма для коммутативных полугрупп решается положительно // Теория моделей и ее применения. Алма-Ата, 1980. С. 75-81.

[98] Тартаковский В.А. О проблеме тождества для некоторых типов групп // Докл. АН CCCP. 1947. T. 58. C. 1909-1910. 
[99] Тартаковский В. А. Решение проблемы тождества для группы с $k$-сократимым базисом при $k=6$ // Изв. АН СССР. Сер. матем. 1949. Т. 13. С. 483-494.

[100] Thompson A. Thin position and the recognotion problem for $S^{3} / /$ Math. Res. Lett. 1994. V. 1. № 5. P. 613-630.

[101] Thue A. Problem über Veränderungen von Zeichenreihen nach gegebehen Regeln // Vid. Skr. Math.-natur. KI. 1914. V. 10.

[102] Tietze H. Über die topologischen Invarianten mehrdimensionaler Mannigfaltigkeiten // Monatsh. Math. Phys. 1908. V. 19. P. 1-118.

[103] Цейтин Г. С. Относительно проблемы распознавания свойств ассоциативных исчислений // Докл. АН СССР. 1956. Т. 107. № 2. С. 209-212.

[104] Цейтин Г. С. Ассоциативное исчисление с неразрешимой проблемой эквивалентности // Докл. АН СССР. 1956. Т. 107. № 3. С. 370-371.

[105] Цейтин Г. С. Ассоциативное исчисление с неразрешимой проблемой эквивалентности // Труды МИАН. 1958. Т. 52. С. 172-189.

[106] Turing A. M. On computable numbers, with an application to the Entscheidungsproblem // Proc. London Math. Soc. (2). 1936. V. 42. № 3, 4. P. 230-265.

[107] Валиев М.К. Примеры универсальных конечно определенных групп // Докл. АН CCCP. 1973. T. 211. C. $265-268$.

[108] Володин И.А., Кузнецов В.Е., Фоменко А.Т. О проблеме алгоритмического распознавания стандартной трехмерной сферы // УМН. 1974. Т. 29. № 5. С. 71-168.

[109] Watier G. On the word problem for single relation monoids with an unbordered relator // Internat. J. Algebra Comput. 1997. V. 7. №6. P. 749-770.

[110] Zhang L. A short proof of a theorem of Adjan // Proc. Amer. Math. Soc. 1992. V. 116. № 1. P. 1-3.

Математический институт им. В. А. Стеклова РАН;

Поступила в редакцию

Ярославский государственный университет им. П.Г. Демидова

26.01.2000

E-mail: si@adian.mian.su, durnev@univ.uniyar.ac.ru 